\title{
Single Molecule Cryo-Fluorescence Microscopy
}

\section{DISSERTATION}

for the award of the doctoral degree in natural science

"Doctor rerum naturalium"

of the Georg-August-Universität Göttingen

within the doctoral program

Physics of Biological and Complex Systems

of the Göttingen Graduate School of Neurosciences, Biophysics,

and Molecular Biosciences (GGNB)

of the Georg-August University School of Science (GAUSS)

submitted by

Weixing Li

from Beijing, China

Göttingen 2016 

Members of the thesis committee:

Prof. Dr. Jörg Enderlein (Referee)

III. Institute of Physics - Biophysics

Georg-August University Göttingen

Prof. Dr. Holger Stark (Referee)

Department of 3D Cryo-Electron Microscopy

Max Planck Institute for Biophysical Chemistry

\section{Prof. Dr. Kai Tittmann}

Schwann-Schleiden Research Center

Department of Molecular Enzymology

Georg-August University Göttingen

Other members of the examination board:

\section{Prof. Dr. Andreas Janshoff}

Institute for Physical Chemistry

Georg-August University Göttingen

\section{Dr. Alexander Egner}

Laser Laboratory Göttingen

\section{Dr. Andreas Neef}

Bernstein Center for Computational Neuroscience Göttingen

Max Planck Institute for Dynamics and Self-Organization

Date of oral examination: 26.10.2016 


\section{Affidavit}

Hereby, I declare that the presented thesis has been written independently and with no other sources and aids than quoted.

Parts of this thesis have been published in:

Li, W., Stein, S. C., Gregor, I. \& Enderlein, J. Ultra-stable and versatile widefield cryofluorescence microscope for single-molecule localization with sub-nanometer accuracy. Opt. Express, 23, 3770-3783 (2015).

Göttingen, 13.09.2016 


\section{Contents}

\begin{tabular}{ll}
\hline ABSTRACT & 1
\end{tabular}

INTRODUCTION 2

I CRYO-FLUORESCENCE MICROSCOPY SYSTEM 12

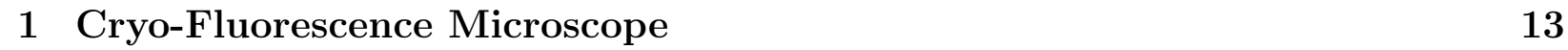

1.1 Cryostat design . . . . . . . . . . . . . . . . . . . . . 13

1.2 Cryostat coupling to microscope . . . . . . . . . . . . . . . . . . . 15

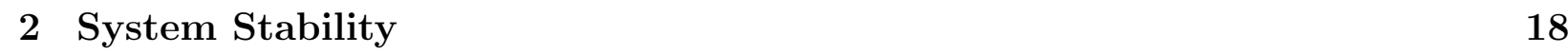

2.1 Thermal stability . . . . . . . . . . . . . . . . . . . . . 18

2.2 Mechanical stability $\ldots \ldots \ldots \ldots \ldots \ldots$

3 Optimization for High Imaging Quality 21

3.1 Choice of proper objective . . . . . . . . . . . . . . . . . 21

3.2 Background minimization $\ldots \ldots \ldots \ldots \ldots \ldots$

3.2 .1 Introduction . . . . . . . . . . . . . . . . . . . . . . . 23

3.2 .2 Background from coverslip . . . . . . . . . . . . . . . . . . . . 24

3.2 .3 Background from sample holder . . . . . . . . . . . . . . . 26

3.2 .4 Background from objective . . . . . . . . . . . . . . . . . 28

3.3 Imaging quality . . . . . . . . . . . . . . . . . . . . 31

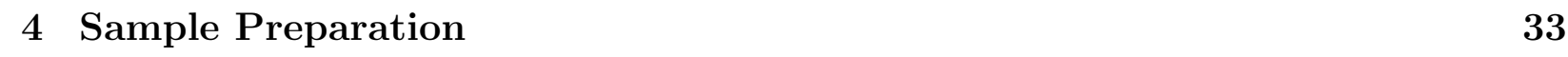

4.1 Introduction . . . . . . . . . . . . . . . . . . . . . . . . . . . . . . . . . . . 33

4.2 Dry mounting . . . . . . . . . . . . . . . . . . . . . . . . . 33

4.3 Polymer medium mounting $\ldots \ldots \ldots \ldots$. . . . . . . . . . . . . . . . . . 34

4.4 Aqueous medium mounting . . . . . . . . . . . . . . . . . . . 35

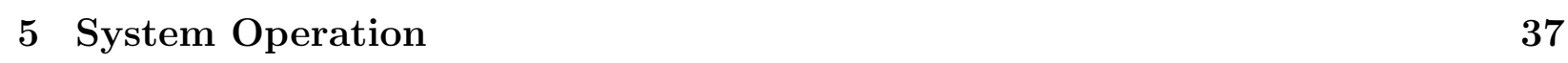

5.1 Sample transfer at RT . . . . . . . . . . . . . . . . . 37

5.2 Sample transfer at CT . . . . . . . . . . . . . . . . . . . . . . . 37

5.3 Gas supply . . . . . . . . . . . . . . . . . . . . . . . 40 
$\begin{array}{lll}6 & \text { Photostability } & 43\end{array}$

6.1 Photostability enhancement at $89 \mathrm{~K} \ldots \ldots \ldots$. . . . . . . . . . . 43

6.2 Photostability at different excitation powers . . . . . . . . . . . . . . 45

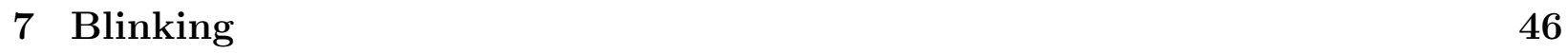

7.1 Introduction . . . . . . . . . . . . . . . . . . . . . . . . . . . . . 46

$7.2 \quad$ Blinking of Alexa647, Atto647N, and Cy5 dyes . . . . . . . . . . . . . . . 47

7.3 Blinking of Atto488 and Alexa488 dyes . . . . . . . . . . . . . . . . . . . . . 49

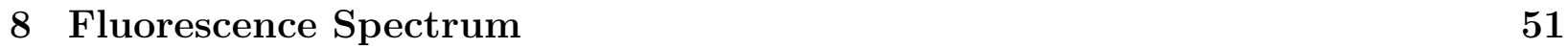

III APPLICATIONS $\quad 53$

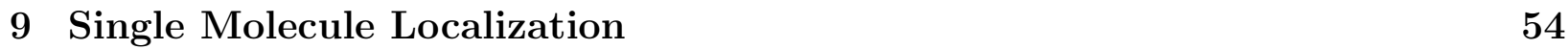

10 Dual Molecule Colocalization 56

10.1 Introduction . . . . . . . . . . . . . . . . . . . . . 56

10.2 Colocalization by separate emission $\ldots \ldots \ldots$. . . . . . . . . . 57

10.3 Colocalization by individual excitation . . . . . . . . . . . . . . 58

10.4 Colocalization by blinking . . . . . . . . . . . . . . . . . 61

10.5 Colocalization by polarization . . . . . . . . . . . . . . . 62

10.5 .1 Theory . . . . . . . . . . . . . . . . . . . 62

10.5 .2 Experimental setup . . . . . . . . . . . . . . . 65

10.5 .3 System calibration . . . . . . . . . . . . . . . . . . . 68

10.5.4 Single molecule polarization microscopy . . . . . . . . . . . . . . . . . 69

10.5 .5 Outlook . . . . . . . . . . . . . . . . . . 70

\begin{tabular}{ll}
\hline CONCLUSION \& OUTLOOK & 71
\end{tabular}

\begin{tabular}{ll}
\hline APPENDIX & 72
\end{tabular}

Coverslip Cleaning Protocol . . . . . . . . . . . . . . . . . . . . . 72

Objective Photon Collection Efficiency _. . . . . . . . . . . . . . . . . . 73

\begin{tabular}{ll}
\hline BIBLIOGRAPHY & 74 \\
\hline
\end{tabular}

$\begin{array}{ll}\text { LIST OF FIGURES } & 82\end{array}$

[LIST OF MOVIES $\quad 84$ 
\begin{tabular}{ll}
\hline ABBREVIATIONS & 87
\end{tabular}

\begin{tabular}{ll}
\hline ACKNOWLEDGMENTS & 89
\end{tabular} 


\section{ABSTRACT}

This work presents a newly developed cryo-fluorescence microscopy system and several of its applications. The thesis is divided into three parts. Part I introduces the design of the cryo-fluorescence microscope, and demonstrates its superior cooling efficiency, mechanical stability, and imaging quality. The high performance of this system allows for continuous single molecule imaging for up to six hours with minor post-correctable drift at $89 \mathrm{~K}$. This system also provides the possibility to transfer vitrified samples at cryogenic temperatures, which enables correlative measurements with cryo-electron microscopy. Part II explores the properties of commonly used organic dyes at $89 \mathrm{~K}$. The photostability is drastically enhanced at liquid nitrogen temperatures, resulting in an average photon yield (detected) of several millions per molecule. The blinking of Alexa647, Atto647N, and Cy5 dyes is slowed down by a factor of ten at $89 \mathrm{~K}$. Atto488 and Alexa488 dyes enter prolonged dark states in the cryostat due to the lack of oxygen (sample located in vacuum in the cryostat), which makes single molecule localization microscopy directly applicable at $89 \mathrm{~K}$. Part III demonstrates single molecule localization with exceptional sub-nanometer precision exploiting the large photon yield at $89 \mathrm{~K}$, and suggests approaches to colocalize two fluorescent molecules with a similar precision. 


\section{INTRODUCTION}

\section{Single (Fluorescent) Molecule Detection}

The detection of single (fluorescent) molecules at cryogenic temperature (CT) started about four decades ago. In 1989, single molecules were detected for the first time by W. E. Moerner via the observation of narrow-line features in the absorption spectrum of crystalline samples kept at liquid helium (LHe) temperature $(4 \mathrm{~K})$ 11. One year later, in 1990, M. Orrit and J. Bernard detected single fluorophores through their emission spectrum with a much higher signal-to-noise $(\mathrm{s} / \mathrm{n})$ ratio, also at LHe temperatures ${ }^{2}$. These are the first moments when people became able to detect single molecules, which are nanometer-sized particles. This revolutionary achievement ended the time of measuring physical entities in ensemble average, and initiated the era of single molecule spectroscopy ${ }^{[3}$ and microscopy ${ }^{[}$.

Concerning the numerous methods for single-molecule detection and its applications ${ }^{[5}[\sqrt{6}$, I would like to highlight three aspects that are related to super-resolution microscopy, and to the work in this thesis.

(i) The possibility/capability of detecting single molecules provides a direct access to studying photophysics on the molecular level $\stackrel{9}{9}$. Astonishing behaviours of single molecules have been discovered in the early 1990s, e.g. blinking 10 and photoswitching [11. These findings lay the foundations for the later application of single-molecule detection to super-resolution microscopy. Also, an enormous step forward was the discovery of fluorescent proteins (FPs), in particular the green fluorescent protein (GFP) which can be photo-switched ${ }^{[2]}$. The photo-switchable FPs made localization-based super-resolution techniques possible with intrinsically expressed fluorophores in living cells..

(ii) Single fluorescent labels have a typical size of a couple of nanometers. This scale is in drastic contrast to the optical diffraction limit, which is several hundreds of nanometers. Thus, fluorescent markers are superb probes to "portray" the structure of samples on the nanometer scale. If the markers are isolated, i.e. only one marker is present within one diffraction-limited observation spot, its position can be determined with a very high accuracy of down to a few nanometers (discussed below). Therefore, single molecule localization/tracking can be used e.g. to study structural details on the nanometer level ${ }^{13}$, or to study the diffusion in membranes and the cytosol 14 . Eventually, the development of controllable photo-switchable fluorophores gave birth to all localization-based super-resolution microscopy techniques $\underline{8}$. 
(iii) Before the development of far-field super-resolution microscopy, resolving structures below the diffraction limit had already been realized by E. Betzig using near-field scanning optical microscopy $(\mathrm{NSOM})^{15 / 16}$. However, as its name infers, NSOM can only be applied to image the surface of samples. The extremely short working distance (focus) of $\sim 10 \mathrm{~nm}$ hinders this technique from being applied to imaging inside biological samples. Besides providing "super-resolution", NSOM also played an imported role at the dawn of the single molecule detection at RT ${ }^{17118}$. When imaging single fluorophores using NSOM, nonsymmetric point spread functions (PSFs) were observed ${ }^{17}$. This is because the fluorophores are immobilized on the surface, and have fixed fluorescence dipole orientations. From their PSF patterns, the nanometric spatial structure of electric fields can be measured, and the dipole orientations can be determined.

\section{The Diffraction-Limited Resolution of Light Microscopy}

The invention of the light microscope in the 16th century opened up a fantastic "microscopic" world that had never seen by humans before. Although it is difficult to identify the inventor of the first microscope, Antonie van Leeuwenhoek is regarded as the first scientifically important microscopist, who made innumerable important discoveries, such as the existence of sperms and eggs, and single-celled organisms such as amoeba and bacteria. He is also credited with popularizing the microscope in the field of biology. Using self-made microscopes, he observed and depicted unicellular organisms about four centuries ago, and named these newly discovered living forms animalcules at that time.

However, no matter how perfectly lenses can be made, the resolution of a light microscope is physically limited by the diffraction of light, restricting it to about half the wavelength along the lateral plane, and even worse in the axial direction, as first described by Ernst Abbe in $1873^{19}$. This is due to the fact that light is an electromagnetic wave, and thus it is subject to interference. An optical microscope is nothing else than a lens system which collects light from a specimen, and focuses it into a magnified image. Because an objective has a finite aperture, it only collects a part of the light emitted from the sample, and the light - as an electromagnetic wave - will interfere when focused into a single point in the image plane. As a result, the light from a dimensionless point emitter cannot be focused to an infinitely small spot in the image plane. Instead, a blurred spot with a much larger size is formed.

The 3D intensity profile of this blurred point-source image is named the point spread function (Fig.11), which determines the resolution of a microscope. In the focal plane, the PSF pattern is also known as the Ariy disc, named after the English mathematician and astronomer 

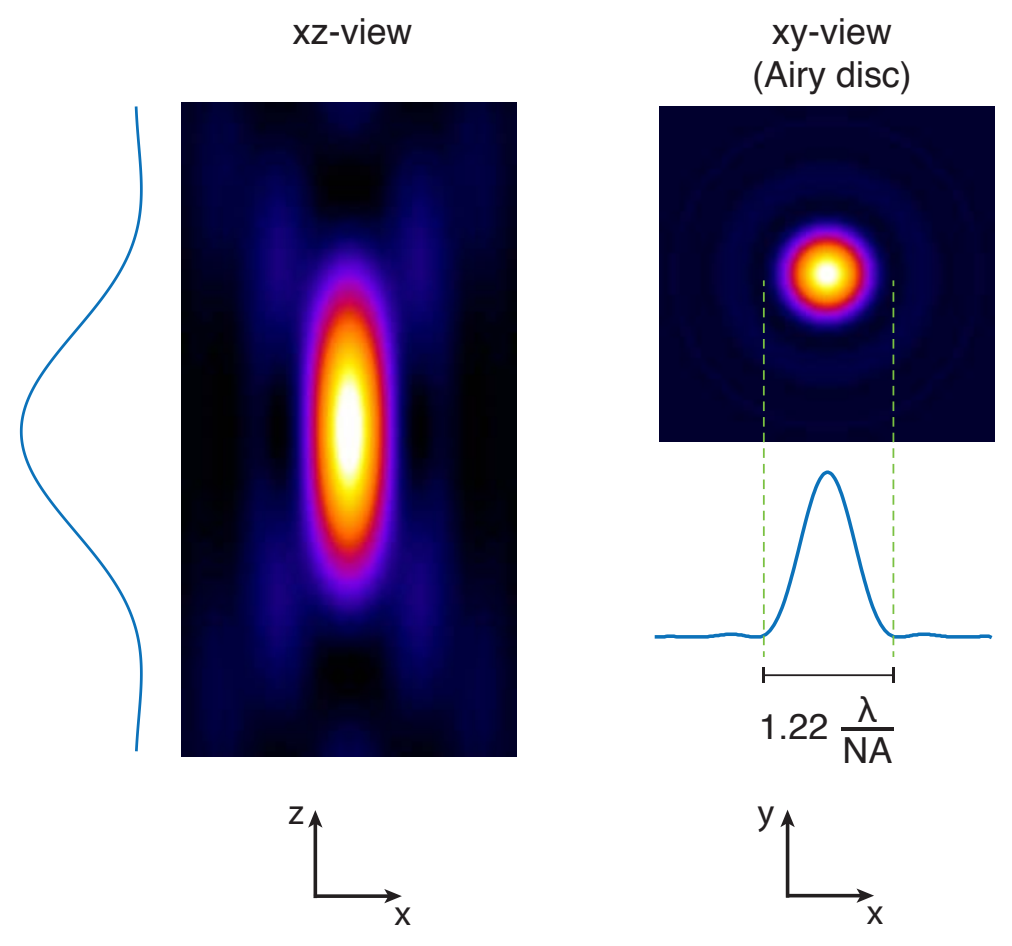

Figure 1: PSF of a conventional light microscope. The PSF of a conventional microscope is displayed by its $\mathrm{xz}$ and $\mathrm{xy}$ cross-sections. The intensity profile in the focal plane is known as the Airy disc. The distance between its first two minima is $1.22 \lambda / \mathrm{NA}$.

George Biddell Airy. The resolution of a microscope can then be defined as the distance between two emitters at which their PSFs can be just separated. Different scientists came up with different criteria for how to define "just separated PSFs", as illustrated in Fig. 2. These criteria differ only slightly by their pre-factors, but are all based on two fundamental factors: the wavelength of the imaged light $\lambda$, and the numerical aperture (NA) of the objective. The $\mathrm{NA}$ is defined as $\mathrm{NA}=n \sin \theta$, where $n$ is the refractive index of the imaging medium, and $\theta$ is the half angle of the light collection cone. It describes the angle over which the objective is able to collect light. For fluorescence microscopy in the visible range (400 - $700 \mathrm{~nm}$ ), and using an oil immersion objective of $1.4 \mathrm{NA}$, the lateral resolution is restricted to about 140 - $250 \mathrm{~nm}$. Along the axial direction, the PSF is elongated even further than laterally, thus the axial resolution is 2-3 times worse. This asymmetry comes from the one-side detection of light when using only one objective, as discussed below.

\section{High-Resolution Microscopy}

Confocal microscopy About four decades ago, it was proposed that the diffractionlimited resolution can be improved by a factor of $\sqrt{2}$ in confocal microscopy 2223]. In this technique, a laser beam is focused into a spot - which is also diffraction-limited - to illuminate 

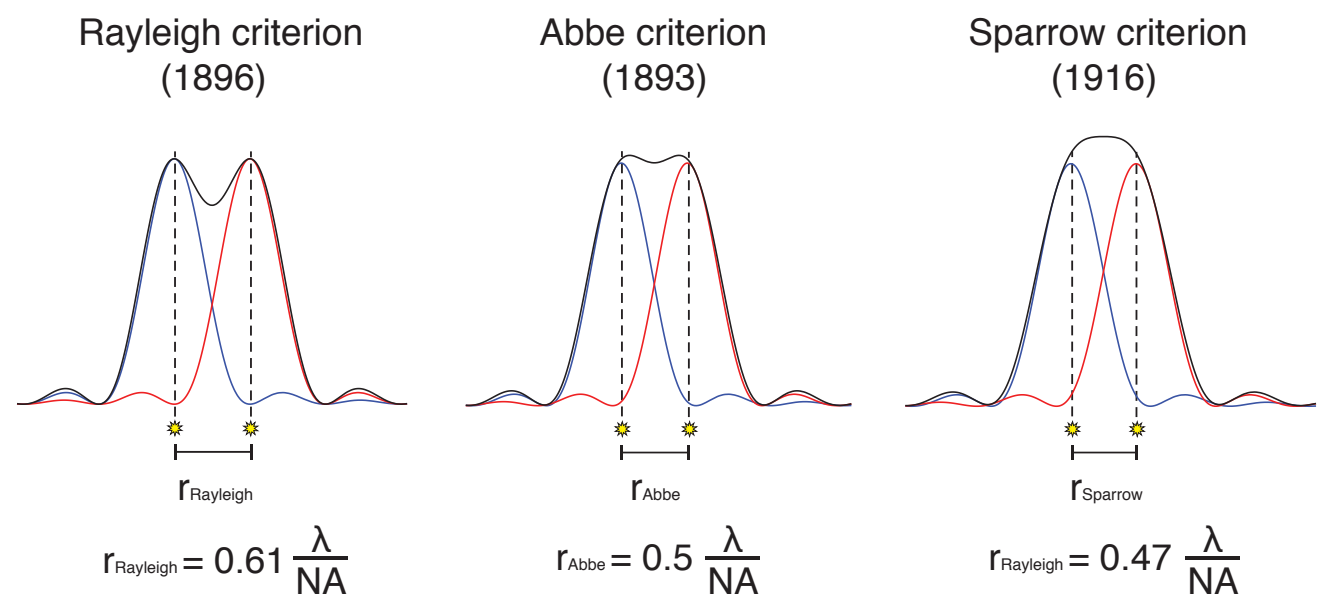

Figure 2: Microscope resolution criteria. In the Rayleigh criterion ${ }^{20}$, the first minimum of one Airy disc falls on the maximum of the second Airy disc. The sum intensity shows a distinct dip. In the Abbe criterion [19], the two Airy discs get slightly closer compared to the Rayleigh criterion, but a dip is still recognizable. In the Sparrow criterion 21, the maxima of the two Airy discs merge to one flat top.

the sample. The collected fluorescence is focused again and sent through a pinhole that is placed exactly at the a plane conjugate to the focal plane in the sample (thus "confocal"). The pinhole geometrically rejects the out-of-focus light from the sample, thus significantly reducing the image background and endowing the confocal microscope with 3D-sectioning capability. In principle, by decreasing the size of the pinhole, the PSF size can also be reduced, resulting in a higher resolution by a maximal factor of $\sqrt{2}$.

However, reducing the pinhole size goes hand-in-hand with rejecting more light, which decreases the detectable signal. In practice, the pinhole cannot be closed to the size at which the predicted $\sqrt{2}$-fold resolution improvement is reached, while maintaining a reasonable $\mathrm{s} / \mathrm{n}$ ratio. Instead, the diameter of the pinhole is typically chosen to be one Airy unit (AU), which is the size of the magnified Airy disc in the confocal plane. Therefore, the purpose of using the pinhole is mainly to suppress the background, to realize three-dimensional sectioning, and to increase the $\mathrm{s} / \mathrm{n}$ ratio, but not to improve the resolution.

Image scanning microscopy The proposed $\sqrt{2}$-fold resolution improvement in confocal microscopy was realized by the development of image scanning microscopy (ISM) 24 by C. Müller and J. Enderlein in 2010. In ISM, the excitation is done the same as in confocal microscopy using a focused laser spot. But in the detection path, the pinhole in confocal microscopy is replaced by a camera, which is a 2D array of photodetectors. Each pixel on the camera chip acts as a pinhole with a much smaller size than one AU, which satisfies the criterion for achieving $\sqrt{2}$ times higher resolution. But the pixels are arranged in different 
positions in the focal plane, and consequently, each pixel "sees" the sample from a slightly different angle. As a result, the scan images recorded by each pixel are shifted with respect to each other. The shifting direction and distance can be precisely determined for each pixel. Thus, these images can be shifted back to reconstruct a final sharp image by data postprocessing. In this way, imaging with a $\sqrt{2}$ times improved resolution and a significantly improved $\mathrm{s} / \mathrm{n}$ ratio is achieved.

As ISM is essentially a scanning microscope, the imaging process is rather slow. To speed up the measurement, parallelization of ISM was realized by using a confocal spinning disc 25 or a digital micromirror array ${ }^{26}$. Furthermore, the rearrangement of the shifted images can be also realized optically. For example, in re-scan confocal microscopy (RCM) 27 - a technique with the same imaging principle as ISM - the positioning of the images to their correct location is carried out by using an additional scan mirror in the detection path. This rescan-mirror reflects the image beam to the required positions on the camera chip, and delivers an image with $\sqrt{2}$ times improved resolution in real-time.

Structured illumination microscopy In confocal microscopy, the excitation is done by focusing a laser beam into a diffraction-limited spot, and scanning the sample with this spot. This illumination carries spatial information in the intensity pattern, which is the fundamental reason why confocal microscopy can theoretically achieve a higher resolution below the diffraction limit. This principle can be generalized to wide-field imaging by extending the pointlike illumination spot to a continuous pattern with defined spatial structure. This idea was conceptualized by M. Gustafsson as structured illumination microscopy (SIM) in 2000 . 28. In SIM, a periodic illumination pattern - typically a sinusoidal wave - is used to excite the sample. Although this illumination pattern itself is also diffraction-limited, its interference with the sample structure creates Moiré patterns, and shifts the high-frequency information of the sample to lower frequencies, which can be detected by the microscope. By taking multiple images with different pattern positions and illumination directions, sample structures below the diffraction limit can be reconstructed with a doubled resolution in the lateral direction.

ISM and SIM are fundamentally equivalent techniques, because they both utilize the structural information of the non-uniform illumination pattern - either a diffraction-limited spot or a sinusoidal wave - to achieve resolution doubling. Compared to ISM, which is a scanning technique, SIM has a higher throughput as a wide-field technique, and its imaging speed is not influenced by the size of the imaging area. The drawback of SIM is its sophisticated setup to create the structured illumination pattern, and the complicated off-line image reconstruction. 
4Pi and $\mathbf{I}^{5} \mathrm{M}$ In conventional microscopy, one objective is used to collect light from one side of the sample, while half of the light is lost on the other side. This is the fundamental reason why the PSF is elongated along the axial direction, resulting in a poorer axial resolution. One straight forward method to solve this problem is to use two objectives opposing each other to collect light from both sides of the sample. This approach was realized in two techniques, known as $4 \mathrm{Pi}^{[29]}$ and $\mathrm{I}^{5} \mathrm{M}^{30[31}$ microscopy. These techniques improve the axial resolution by up to sevenfold down to $\sim 100 \mathrm{~nm}$.

\section{Super-Resolution Microscopy}

Imaging far below the diffraction limit was realized by the development of a series of farfield microscopy techniques, often referred to as "super-resolution" microscopy, or simply "nanoscopy". These techniques achieve a typical lateral resolution of several tens of nanometers, which is one order of magnitude below Abbe's diffraction limit. The "super-resolution" enables direct observation of samples on the nanometer scale, and has resulted in numerous applications and discoveries in the chemical, biological, and medical sciences ${ }^{32}$ 34. Thus, the Nobel Prize for Chemistry 2014 ${ }^{35}$ was awarded to the pathfinders of these techniques - Eric Betzig, Stefan W. Hell, and Willian E. Moerner. Super-resolution microscopy has been extensively reviewed in the literature ${ }^{34}$. In this thesis, I would like to highlight two (groups) of these techniques, which are related to the work in this project.

Stimulated emission depletion microscopy (STED) In confocal microscopy, the excitation laser cannot be focused into an infinitely small spot due to diffraction. The actual illumination extent is given by the size of the PSF (Fig.11), which is typically $\sim 200 \mathrm{~nm}$ in the lateral direction, and $\sim 500 \mathrm{~nm}$ in the axial direction. When illuminating a sample with this PSF, all fluorophores within the focal volume will be excited, and will emit fluorescence at the same time. Thus, structures within the diffraction-limited spot cannot be separated.

In 1994, S. Hell conceived a technique to reduce the effective excitation volume in confocal microscopy, referred to as stimulated emission depletion microscopy (STED) 39140 . In STED, an additional laser spot with a doughnut-like intensity profile is overlapped with the confocal illumination spot. This doughnut spot has a zero intensity in the middle, and its wavelength matches the transition energy of the fluorophore from the excited state to one of the vibrational energy levels in the ground state. When shining this STED beam onto the fluorophores that are exited by the confocal beam, the fluorophores will be induced to return exactly to that energy level, thus will emit fluorescence exactly at that wavelength. When choosing this wavelength further away from the emission maximum of the fluorophore, the induced fluorescence (stimulated emission) together with the STED laser can be filtered out, 
leaving only the fluorophores located in the zero center of the doughnut beam detectable. The size of this zero center is typically by one order of magnitude smaller than the size of the confocal volume, which determines the resolution of STED.

The "super-resolution" in STED originates from discrete quantum-mechanical nature of fluorescence excitation and emission. But in my view, it does not, in any way, break the fundamental physical law of Abbe's diffraction limit, because the confocal beam as well as the STED beam are both diffraction-limited. The achieved effective super-resolution highly relies on the quantum-mechanical nature of fluorescence, described in its simplest form by a two-state system which can be resonantly excited and de-excited.. This super-resolution can only be achieved for a sub-class of optical microscopy, namely fluorescence microscopy, and in a very particular case when the used dyes are STED-compatible. But the fundamental physics behind it, as described by Abbe, remains unchanged.

Single molecule localization microscopy (SMLM) Unlike STED, which is a technique based on ensemble fluorophore imaging, another group of super-resolution techniques, referred to as single molecule localization microscopy (SMLM), utilizes the localization of single fluorescent molecules to achieve a higher resolution below the diffraction limit. Single fluorophores have a size of $\sim 1 \mathrm{~nm}$ for organic dyes, and $\sim 4 \mathrm{~nm}$ for fluorescent proteins. But due to diffraction, these point sources are imaged as much larger spots with a size of several hundred nanometers, as described by their PSFs (Fig. 1). However, if only one molecule is present in the diffraction-limited spot, the actual molecule's position, i.e. the center position of its image, can be determined with a much higher accuracy than the size of the PSF (by fitting the PSF with a Gaussian function).

However, a biological sample is usually labeled with fluorophores at a very high concentration. There are typically hundreds of fluorescent markers located in one diffraction-limited spot. If these markers emit light simultaneously, their PSFs overlap, resulting in a smeared out image. But if these markers can be made fluorescent sequentially one after another, they can be then localized separately with very high accuracy. Displaying these localizations will create an image with a resolution below the diffraction limit. This super-resolution idea was proposed first by E. Betzig in 1995, and was implemented by himself in 2006 using photoactivatable fluorescent proteins (PA-FPs), naming the technique photoactivated localization microscopy (PALM) 41]. Almost simultaneously (one day before the PALM publication), another fundamentally equivalent method was published independently by X. Zhuang, named stochastic optical reconstruction microscopy (STORM) 42. Later the same year, another similar technique was demonstrated by S. Hess, named fluorescence-PALM (fPALM) 43 . 
These three independent methods share the same principle. In wide-filed, the fluorophores are photoactivated, or photoswitched from the dark state to the fluorescent state sparsely. The sparsity is controlled by optical or chemical means to the level where the PSFs of the "on" molecules do not overlap on each frame of exposure. In this way, the molecules located in the diffraction-limited spot are separated temporally. When sequentially imaging all the molecules, and localizing them individually on each frame, an image can be reconstructed by overlapping all the localizations, and the resolution is determined only by the localization accuracy. Given Abbe's resolution limit (PSF size) $\delta_{\text {Abbe }}$, the localization accuracy $\delta_{l o c}$ is determined by the detected number of photons $N$ per molecule during each exposure in the form of $\delta_{l o c} \propto \delta_{A b b e} / \sqrt{N}$. In practice, several hundred photons can be detected from single molecules in a typical integration time of tens of milliseconds. This results in a localization accuracy/resolution of several tens of nanometers in the lateral plane in SMLM.

\section{Cryo-Fluorescence Microscopy}

Although super-resolution microscopy techniques have theoretically unlimited resolution, the practically achievable resolution is restricted to several tens of nanometers. The main reason for this limitation is the unavoidable photo-bleaching of the fluorophore, which restricts the photon yield per molecule. However, it has been known that the photobleaching can be strongly suppressed at $\mathrm{CT}^{4445}$, and millions of photons can be detected from single organic dye molecules $\underline{46}$. Therefore, cryo-fluorescence microscopy (cryo-FM) has drawn great attention after the development of detecting single molecules in the 1980's.

In recent years, cryo-FM has been increasingly applied in the field of super-resolution microscopy ${ }^{47}[49]$, single molecule spectroscopy $\stackrel{50151}{ }$, and correlative light and electron microscopy (CLEM) 45/52/53. Many super-resolution techniques have been shown to be applicable at CT, including cryo-STED ${ }^{47}$, cryo-PALM ${ }^{49 \mid 54}$, etc. Benefiting from the drastically enhanced photostability of the fluorophores, colocalization of two molecules with single nanometer resolution was demonstrated at liquid nitrogen (LN2) temperatures ${ }^{48}$. When combining these techniques with cryo-electron microscopy, cryo-CLEM benefits from the specific labeling in fluorescence microscopy (FM) and the high resolution in electron microscopy (EM), providing a direct way to investigate biological structures and their functions on the

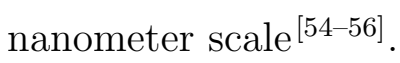

\section{Cryo-Microscopy Systems}

The most crucial component of a cryo-imaging system is a dedicated cryostat. Different types of cryostats have been developed to satisfy specific requirements of various applica- 
tions. For example, in cryo-CLEM, the sample needs to be vitrified before the experiment using rapid freezing techniques 57158 . Therefore, cryostats for cryo-CLEM must provide the possibility to transfer frozen samples below the glass transition temperature of water $(\sim 135 \mathrm{~K})$ to prevent ice crystal formation in the sample $\stackrel{59}{ }$. To meet this requirement, the existing cryostats $\frac{45|52| 60,62}{62}$ are typically designed as an "open system", where the sample is embedded in a cold nitrogen atmosphere for the ease of sample transfer. In these cryostats, the low temperature is sustained by the circulation of LN2 using an attached piping system. This design has several drawbacks. First, purging the cold nitrogen gas through the sample chamber introduces great mechanical instability, which causes large sample drift and vibration. Secondly, the objective is directly exposed to the cold optical window or to the frozen sample, which introduces considerable thermal stress on the objective, and results in large optical aberrations ${ }^{63}$. Thirdly, ice condensation occurs from time to time on the sample or on the inner surface of the optical window due to imperfect dehydration of the working environment. Also, the outer surface of the cold window is often covered by water or ice in a humid lab, which blocks the observation of the sample. These drawbacks limit the reliability of these cryostats, and hinder their applications in accurate and sensitive experiments.

For single molecule microscopy $47 \mid 48$ and spectroscopy ${ }^{50151}$, the cryostats are typically designed to be vacuum-insulated to provide high thermal and mechanical stability $46 / 50 / 64$. In these devices, the sample is typically attached to a cold substrate, which is kept at CT by the circulation of cryogen ${ }^{[8]}$ or by a static cryogen reservoir [50164. The drawback of this design is that any vibration or bubbling of the cryogen will be directly transmitted to the sample, causing considerable mechanical instability. Although the use of LHe instead of LN2 provides a more stable flow and less bubbling, the required recycling system and the helium consumption significantly increase the equipmental and operational cost. Another major limitation of these vacuum-insulated cryostats is that the sample cannot be transferred into/from the cold cryostat. Instead, the sample has to be loaded at RT, and then gradually cooled down. This makes the study of the vitrified samples and the preservation of the examined samples impossible using these devices.

\section{About This Thesis}

To overcome the above-mentioned limitations, a new cryo-fluorescence microscopy system was developed in this Ph.D. project. This new system combines the high thermal and mechanical stability for single molecule imaging with the possibility of cryo-sample transfer for CLEM. This system and its applications are introduced in three parts in this thesis.

In the first part, the design philosophy and the key features of the cryo-fluorescence micro- 
scope are summarized. The newly-developed cryostat is a vacuum-insulated, stand-alone device using static LN2 cooling. One unique feature is that the sample is mechanically decoupled from the unstable cooling source, providing an exceptional high mechanical stability. This enables long-time observation of the sample for up to six hours with negligible drift. Moreover, this cryostat allows for the use of any air objective with a working distance above $1.2 \mathrm{~mm}$. The objective is located outside the cryostat at RT, and the thickness of the optical window can be adapted by the coverslip thickness correction collar of the objective. This results in superior single molecule imaging quality with negligible spherical aberration and field distortion. Furthermore, the sample can be transferred into/from the cold cryostat using a new workflow, which makes CLEM possible with this system.

In the second part, the properties of commonly used organic dyes are examined at $89 \mathrm{~K}$. It is found that the photostability of organic dyes is boosted by more than two orders of magnitude at $89 \mathrm{~K}$, resulting in an average photon yield (detected) of several millions per molecule. Moreover, the blinking of Alexa647, Atto647N, and Cy5 dyes is slowed down by a factor of ten, to an on/off ratio below 0.5. The triplet state of Atto488 and Alexa488 dyes is prolonged drastically in the absence of oxygen in the cryostat, and stochastic photoswitching is achieved in the cryo-conditions.

In the last part, single molecule localization is demonstrated with an exceptional precision below one nanometer at $89 \mathrm{~K}$. One direct application of this result is to colocalize two molecules with single nanometer resolution. One approach has been presented by Weisenburger et al. ${ }^{48}$ However, the authors also reported a very low success rate of this approach $(\sim 0.1 \%)$ with limited reproducibility. In this thesis, the challenges for dual-molecule colocalization with nanometer resolution are discussed, and several different approaches are proposed. 


\section{Part I}

\section{CRYO-FLUORESCENCE MICROSCOPY SYSTEM}

This part of the thesis introduces a new cryo-fluorescence microscopy system developed in this project. Section 1 describes the design philosophy and the structural parts of the cryostat. Section 2 evaluates the system's performance in terms of mechanical and thermal stability. Section 3 presents system optimization to achieve high imaging quality for single molecule imaging. Section 4 discusses different strategies for sample preparation. Section 5 gives operating instructions for sample transfer at RT/CT and gas supply to the sample. 


\section{Cryo-Fluorescence Microscope}

\subsection{Cryostat design}

The core component of a cryo-fluorescence microscope is a dedicated cryostat. In this project, a new cryostat was designed to overcome the limitations of the existing devices. This cryostat is a vacuum-insulated dual-vessel device (Fig. 3a). An inner vessel serves as the cryogen tank, which holds up to $\sim 1.5 \mathrm{~L} \mathrm{LN2/LHe.} \mathrm{This} \mathrm{inner} \mathrm{vessel} \mathrm{is} \mathrm{sealed} \mathrm{into} \mathrm{an} \mathrm{outer} \mathrm{vessel,} \mathrm{which}$ acts as the vacuum housing. Between these two vessels, a vacuum of $\sim 10^{-3} \mathrm{~Pa}$ is generated for thermal insulation. The sample and the internal components of the cryostat are all located in this vacuum.
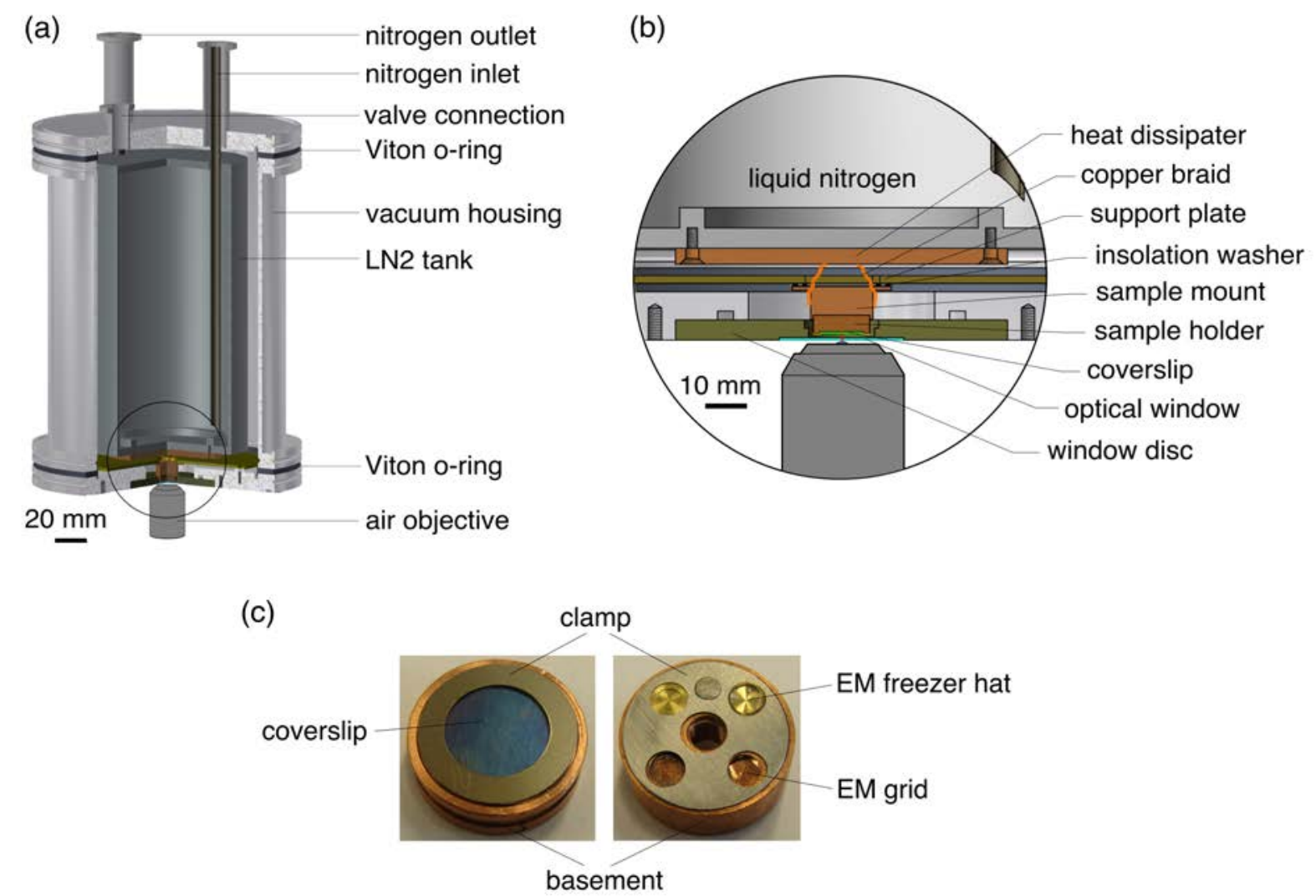

Figure 3: Cryostat design. (a) Cut-away view of the cryostat, accommodating an air objective. (b) Zoom-in view of the central parts of the cryostat (black circle in a). (c) Photos of two types of sample holders for coverslip and EM grids/freezer hats.

Heat dissipater The cryogen tank is made of stainless steel, which has a relative low thermal conductivity at CT (Fig.4). To achieve more efficient thermal transfer, a disc (named "heat dissipater") made of oxygen-free copper (OFC) is attached to the bottom of 
the tank (Fig. 3b). This OFC disc has high thermal conductivity at CT, thus assists with the heat dissipation to the tank through the large contacting area. Between the heat dissipater and the tank, an indium sheet is sandwiched to fill any surface irregularity to assure seamless heat transfer.

Support plate The tank is soldered on the top of the inlet and outlet channels of the vacuum housing, and "hangs" in the free space in the cryostat. By cooling, the tank undergoes great thermal drift and mechanical vibration. Therefore, it is not preferred to fix the sample directly onto the heat dissipater on the bottom of the tank. Instead, an additional "support plate" is created to hold the sample. This plate is fixed on the bottom of the vacuum housing using Teflon spacers and polyimide screws, which have very low thermal conductivities at CT (Fig. 4). This minimizes the heat loss through the vacuum housing. To prevent that the tank vibrations are transmitted to the support plate through the vacuum housing, Viton o-rings were used to seal the parts. Viton is an over-damping material that absorbs vibration when firmly compressed. As a result, the support plate is mechanically decoupled from the unstable tank, and provides a steady place to fix the sample.

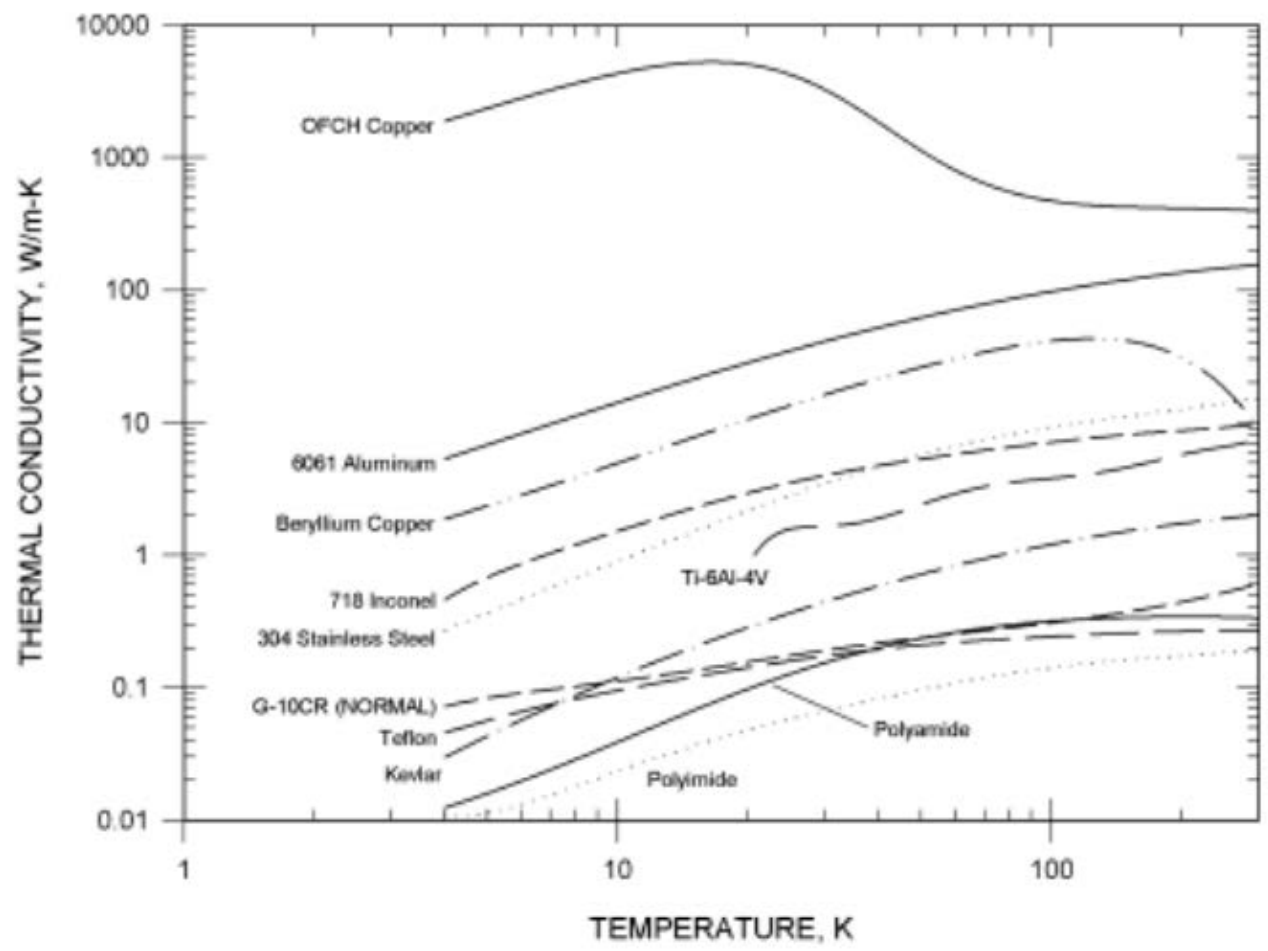

Figure 4: Thermal conductivity of various materials. Reproduced from the publication by Marquardt et al. $[65$

Sample mount To create a substrate to hold the sample, the "sample mount" is constructed, and is fixed on the support plate via thermal insulation washers/screws made of 
polyimide. This sample mount is connected to the heat dissipater via four flexible copper braids. These braids do not only transfer heat and cool down the sample mount, but simultaneously absorb also vibrations and thermal drifts of the tank. This mechanical decoupling of the sample mount from the unstable tank is the key feature that assures a superior mechanical stability.

Sample holder Two types of sample holders have been designed to adapt one coverslip or four EM grids/freezer hats (Fig. 3 c). The sample holder is loaded onto the sample mount through a circular opening on the bottom of the vacuum housing, and is held in position by integrated magnets.

Window disc The opening at the bottom of the cryostat is closed by a "window disc", and is sealed via a Viton o-ring. In the middle of the disc, a $0.5 \mathrm{~mm}$ thick quartz optical window is glued, through which the sample can be observed. After the window disc is firmly closed (via clamps), there is a $\sim 0.5 \mathrm{~mm}$ distance between the sample and the inner side of the optical window. This design allows for the use of any air objective with a working distance above $\sim 1.2 \mathrm{~mm}$, but hinders the use of immersion objectives.

Thermal shield To achieve a high thermal insulation, the cryogen tank, the sample mount, and the sample holder are shielded from external thermal radiation by reflective metal sheets (not shown in the figure).

\subsection{Cryostat coupling to microscope}

The cryostat is designed to be adaptable to any inverted microscope. However, due to its large weight of $\sim 15 \mathrm{~kg}$, an additional supporting stage is preferred to assure high mechanical stability. In this project, a robust motorized $\mathrm{x}-\mathrm{y}$ stage was constructed to support the cryostat (Fig.5a). Below this stage, a conventional inverted epi-fluorescence microscope was assembled. In principle, this stage can be resized to adapt to any commercial microscope.

The cryostat is kept in position on the $\mathrm{x}-\mathrm{y}$ stage via three kinematic mounts. The high precision of these mounts and the large weight of the cryostat deliver a sufficiently high mechanical stability without the need of any additional fixation (e.g. via clamping or screwing). This allows for easy detachment of the cryostat from the stage, and makes the sample change very convenient.

To translate the field-of-view (FOV) across a sample, and to find a suitable position for microscopic observation, a motorized $\mathrm{x}-\mathrm{y}$ stage moves the whole cryostat laterally with respect 
to the objective. Solid mechanical design and a ball bearing rail system assure smooth and accurate displacement of the heavy cryostat. To adjust focusing, the objective is mounted on a vertical piezo stage, and the piezo is mounted on a motorized z-stage for fine and coarse focusing, respectively.

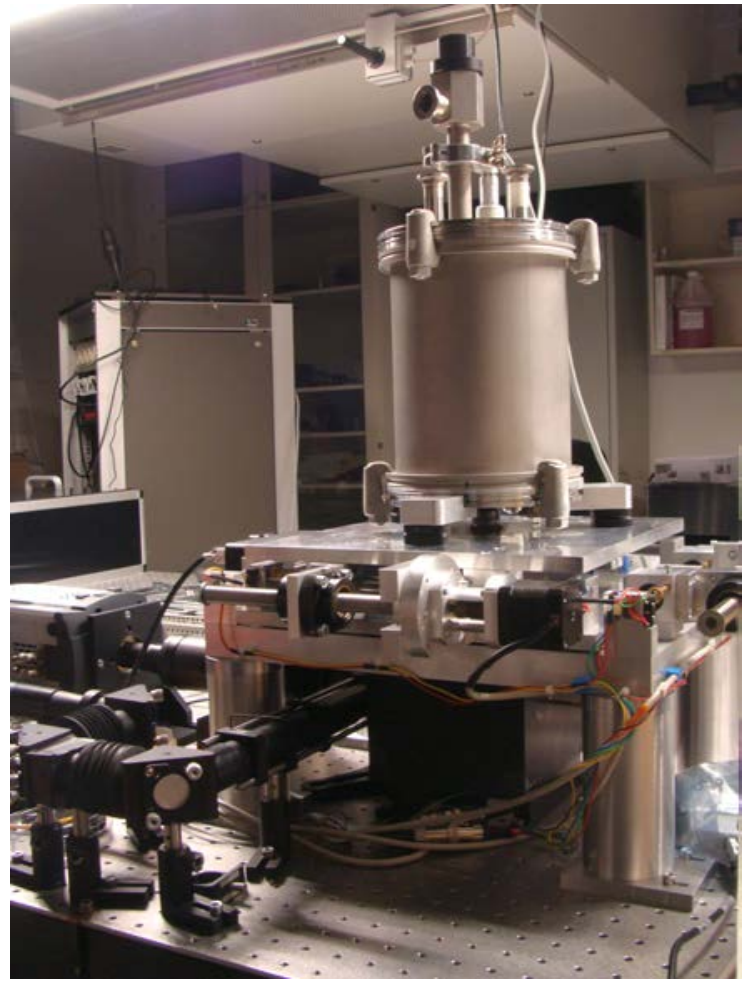

(a)

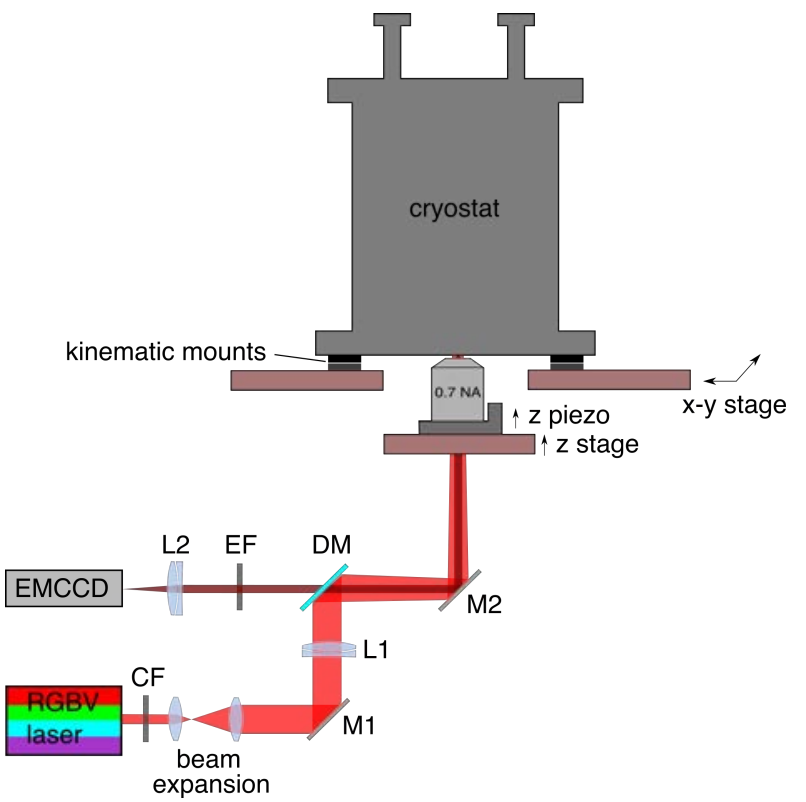

(b)

Figure 5: Cryo-fluorescence microscope. (a) A photo of the cryostat standing on a custombuilt inverted epi-fluorescence microscope. (b) Schematic of the cryo-fluorescence microscope. The cryostat is kept in position on a motorized x-y stage via three kinematic mounts (KBS98, Thorlabs GmbH, Germany). The objective (Olympus LUCPLFLN 60×/0.7 NA) is mounted on a z-piezo actuator (PIFOC, Physik Instrumente GmbH, Germany), which is fastened on a motorized zstage for focusing. Four CW lasers (R-637 nm, G-532 nm, B-473 nm, V-405 nm, Changchun New Industries Optoelectronics Tech. Co., Ltd., China) are used for sample illumination. The laser light is sent through a clean-up filter CF, expanded by a telescope, and focused onto the back focal plane of an air objective by an achromatic lens L1, upon the reflection on a dichroic mirror DM and an adjusting mirror M2. The fluorescence emitted from the sample is collected by the same objective, sent through an emission filter EF, and focused onto an EMCCD camera (iXon Ultra 897, Andor Technology) by a tube lens L2.

A wide-field epi-fluorescence microscope is constructed below the $\mathrm{x}-\mathrm{y}$ stage. This microscope is equipped with four continuous wave (CW) lasers for sample illumination, a high sensitivity electron multiplying CCD (EMCCD) camera for single molecule detection, and an air ob- 
jective with correction collar for high quality imaging. The tube lens can be easily changed to any focal length to provide any desired magnification. The lasers, the motorized filter wheel, and the camera are controlled using a custom-written LabVIEW software (Fig.6). This software also provides basic programmability for the users to define specific sequences of hardware action (e.g. to conduct multi-color imaging).

Beside wide-field microscopy, one could also perform confocal microscopy can also be realized in the cryostat by using objective scanning or laser galvo scanning. Recently, low temperature compatible piezo stages have become available. These stages can be placed inside the cryostat to hold the sample, which would enable sample scanning as well.

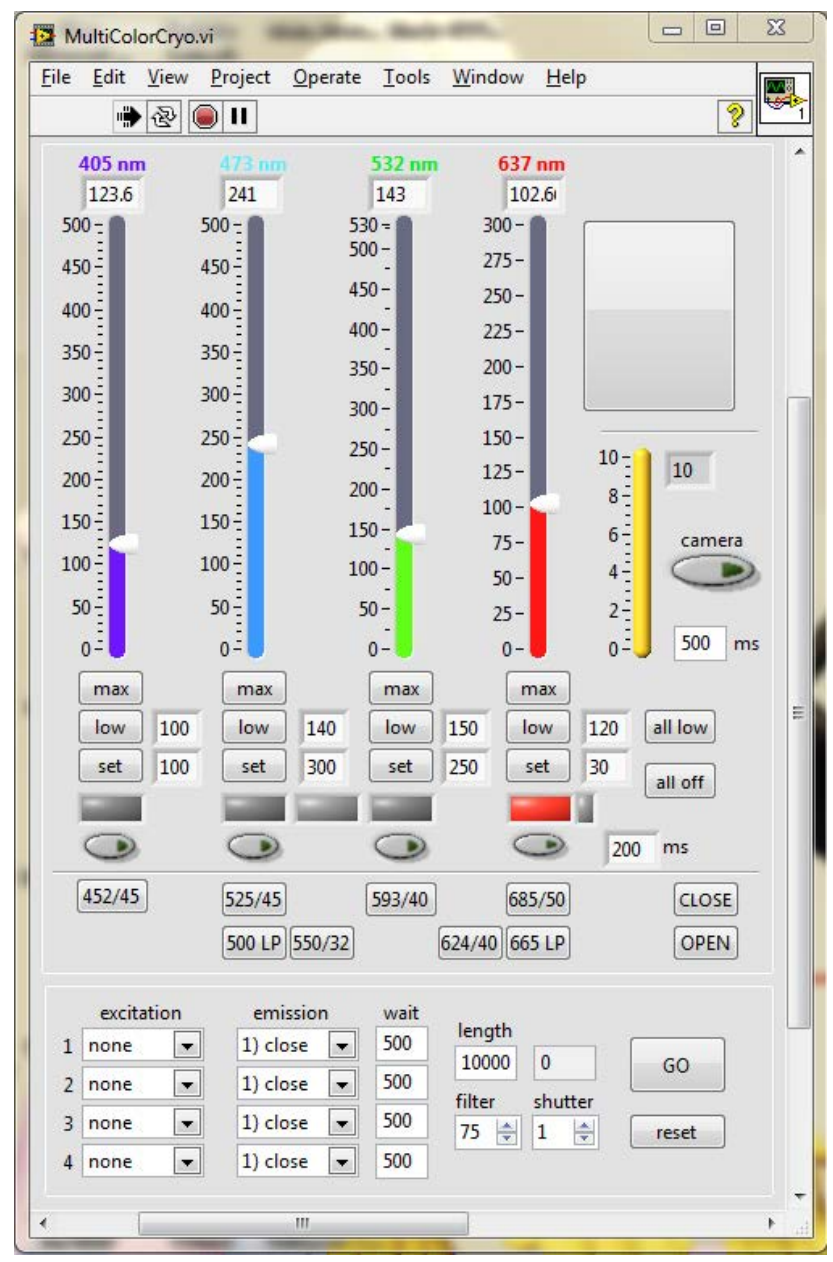

Figure 6: Microscope controlling software. A custom-written LabVIEW software controls the laser switch, the laser power, the emission filter wheel, and the camera trigger. Four combinations of these controls can be defined by the user to conduct a specific sequence of actions automatically. 


\section{System Stability}

\subsection{Thermal stability}

To examine the thermal stability of the system, temperatures of the essential parts of the cryostat were monitored in a cooling test (Fig. 7). Prior to cooling, the air in the cryostat was evacuated using a turbo pump (HiCube, Pfeiffer Vacuum GmbH, Germany), creating a vacuum of $\sim 10^{-2} \mathrm{~Pa}$ (measured by the internal barometer in the turbo pump). Then, LN2 was fed into the tank through a transfer line by the inner pressure of the dewar. The rapidly cooled tank acted as a "molecule catcher", which further reduced the vacuum to $\sim 10^{-3} \mathrm{~Pa}$. After the tank was filled, the vacuum pipe and the transfer line were detached from the cryostat. During the test, the outlet channel of the tank was kept open to release the gently evaporating nitrogen gas.

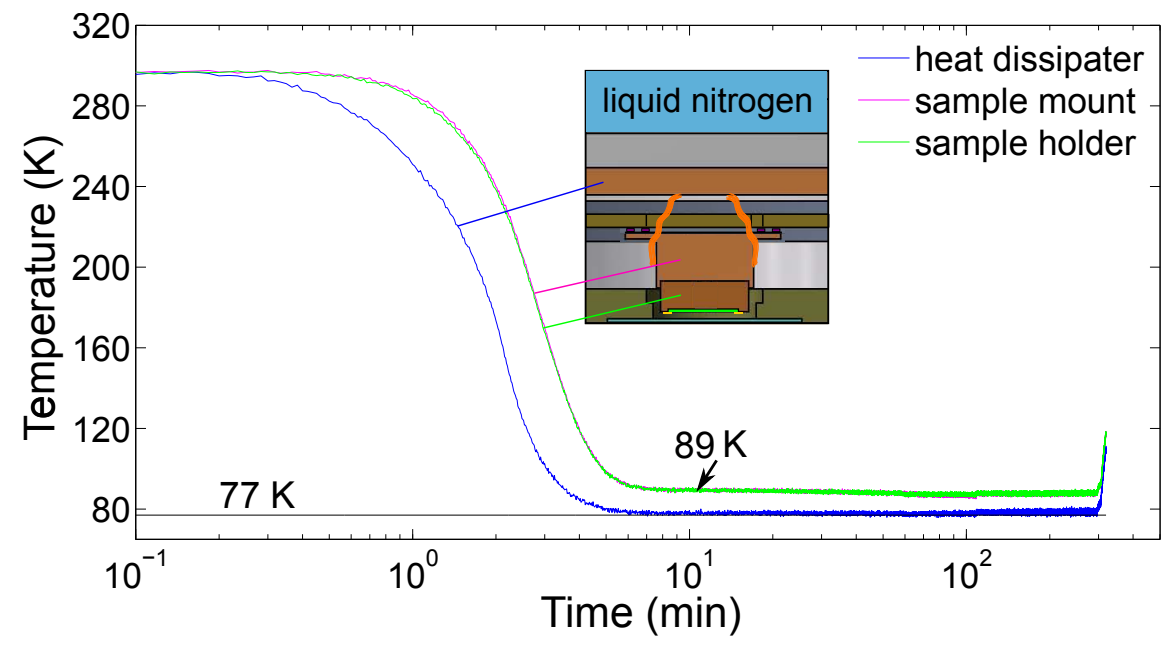

Figure 7: Cryostat cooling test. Temperatures of the heat dissipater, the sample mount, and the sample holder were measured simultaneously at $2 \mathrm{~Hz}$ using embedded thermal sensors (PT100 S234PD12, Telemeter Electronic GmbH, Germany) for over six hours. The cooling test started at RT with empty tank, followed by tank filling with LN2, and was terminated when all LN2 was evaporated (indicated by the temperature rise).

As shown in Fig. 7, the temperature of the heat dissipater dropped from RT (294 K) to the boiling point of LN2 $(77 \mathrm{~K})$ within six minutes. The temperature of the sample mount and the sample holder followed this drop closely, and reached a thermal equilibrium at $89 \mathrm{~K}$ after about eight minutes. Then, all the temperatures remained steady for more than six hours with negligible thermal fluctuation $(<0.1 \mathrm{~K})$.

The fast cooling rate of more than $0.5 \mathrm{~K} / \mathrm{s}$ demonstrates an excellent cooling efficiency 
through the four copper braids. The time of less than ten minutes to reach the thermal equilibrium is exceptional among all existing cryostats. This enables an almost instant start of the cryo-measurement after filling the tank. The identical temperature of the sample mount and the sample holder indicates a seamless heat transfer through the physical contact between these two parts. The high thermal stability over several hours allows for continuous long-lasting experiments. The working time can be limitlessly extended by refilling the tank. The LN2 consumption of $\sim 1.5 \mathrm{~L}$ for six hours is very economic compared to other cooling schemes based on cryogen circulation or purging.

During the test, no ice condensation occurred on the optical window or on any outer surface of the cryostat. This indicates an excellent thermal insulation by the vacuum, which was able to hold a temperature gradient of $\sim 200 \mathrm{~K}$ over a distance of $\sim 0.5 \mathrm{~mm}$ between the sample and the optical window. Overall, this test demonstrates superior cooling efficiency and thermal stability of the cryostat.

\subsection{Mechanical stability}

Mechanical stability of a microscope typically translates into image stability. To quantify the mechanical stability of the cryo-fluorescence microscope, fluorescent beads were imaged in the cryostat at $89 \mathrm{~K}$ for one hour, and their positions were tracked to determine the lateral sample drift (Fig.8).

The positions of the beads were determined by fitting their images (Fig. 8 a inset) to 2D Gaussian functions using the quickPALM ${ }^{66}$ routine in ImageJ ${ }^{67}$. The high brightness of these beads provided high localization precision of a few nanometers per frame. This measurement was repeated at three different positions on the same sample, several millimeters away from each other. During the experiment, the vacuum pipe and the LN2 transfer line were detached from the cryostat, leaving it as a stand-alone device decoupled from external mechanical perturbations.

As shown in Fig.8, the sample underwent a total drift of less than one micrometer in one hour. Position 2 and 3 shared a similar drift profile: a fast relaxation within the first $\sim$ ten minutes, followed by small fluctuations of several tens of nanometers. At position 1, the image continuously drifted away in one hour. This was because position 1 was further away from the geometric center of the sample as compared to position 2 and 3. This suggests that imaging closer to the sample center results in less drift, which is thus preferred. 
(a)

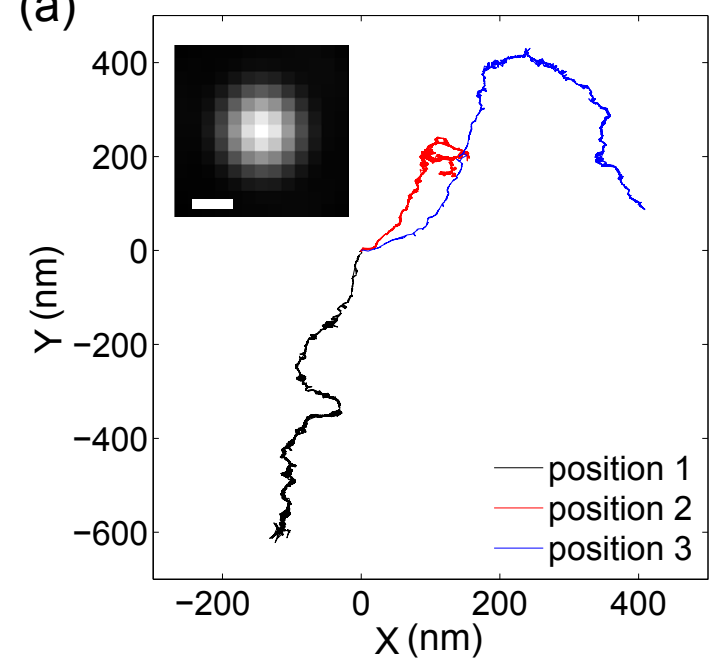

(b)

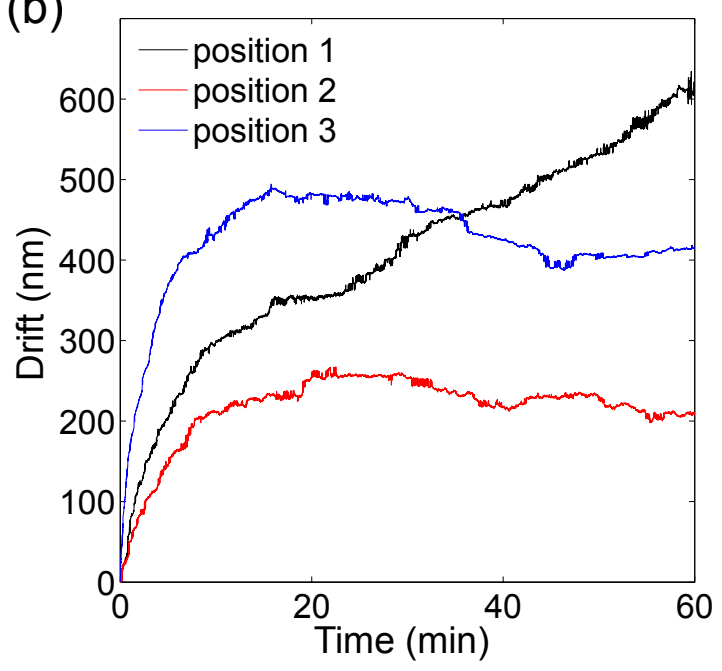

Figure 8: Lateral sample drift. (a) 2D trajectories of the center positions of three fluorescent beads (Life Technologies GmbH, Germany) measured for one hour. A bead image (inset, scale bar $500 \mathrm{~nm}$ ) was taken with $0.4 \mathrm{~s}$ exposure time at $2 \mathrm{~Hz}$ using an EMCCD camera. (b) Lateral sample drift is quantified as the absolute distance to the starting point.

The lateral sample drift is probably due to the local heating of the sample holder upon laser irradiation, because the drift amplitude became larger when increasing the laser power. However, the embedded thermal sensors did not register any global temperature change during the test. But the local heating on the coverslip should be rather minimal due to the efficient heat dissipation of the OFC and the high transparency of the coverslip. This minor lateral drift can be completely corrected by software in post-processing (section 9), thus it is tolerable for most of the experiments.

Another interesting observation was that no visible defocusing of the image occurred during the one hour test, nor in other long-time measurements over more than four hours (section 91. This indicates that the system also has an excellent mechanical stability in the vertical direction.

All in all, the cryo-fluorescence microscope exhibits excellent mechanical stability in all three dimensions as compared to other devices. This is mainly due to the successful mechanical decoupling of the sample from the unstable cooling source, through the use of flexible metal braids and the vibration-absorbing Viton o-rings. The high mechanical stability allows for long-time investigations of samples over several hours without active drift correction. 


\section{Optimization for High Imaging Quality}

\subsection{Choice of proper objective}

The cryostat only allows for the use of air objectives with a working distance above $\sim 1.2 \mathrm{~mm}$, because there is a $\sim 0.5 \mathrm{~mm}$ gap and a $0.5 \mathrm{~mm}$ thick optical window between the sample and the objective. The thick window that is made of quartz introduces considerable spherical aberration to the image. To correct for this aberration, air objectives with an adjustable collar for coverslip thickness correction are preferred. These objectives see the optical window as a "thick coverslip", and can compensate the optical impact of the window thickness by adjusting the collar, hence compensate for the spherical aberration.

Integrating a correction collar into an objective typically limits its NA to 0.7 due to mechanooptical design restrictions. If objectives are constructed without the correction collar, a higher NA of up to 0.9 can be achieved without sacrificing the working distance. However, high NA and correction collar cannot be realized in the same objective. To make a trade-off between the correction collar and the higher NA, the imaging quality was compared between a 0.7 NA Olympus objective with correction collar and a 0.9 NA Nikon objective without correction collar.

Using these two objectives, single Atto647N molecules were imaged in the cryostat through the $0.5 \mathrm{~mm}$ quartz window. For the Olympus $60 \times$ objective, when adjusting the collar to an appropriate value, single molecules were clearly resolved (Fig.9 a). For the Nikon 100× objective, despite its larger NA of 0.9 , single molecules could not be identified when using its original magnification. After adjusting the magnification to $\sim 56 \times$ (by replacing the tube lens of $\mathrm{f}=180 \mathrm{~mm}$ with an achromatic lens of $\mathrm{f}=100 \mathrm{~mm}$ ), single molecules became visible (Fig.9]b). The imaging quality in terms of $\mathrm{s} / \mathrm{n}$ and signal-to-background (s/b) ratios was similar for these two objectives at comparable magnifications.

But a 0.9 NA objective has about a doubled photon collection efficiency as compared to a 0.7 NA objective, according to the formula (plotted in Fig. 10, derivation see appendix 10.5.5):

$$
I \propto 2 \pi\left[1-\sqrt{1-\left(\frac{\mathrm{NA}}{n}\right)^{2}}\right]
$$

where $I$ is the photon collection efficiency, and $n$ is the refractive index. Despite the higher NA, the Nikon objective does not deliver higher $\mathrm{s} / \mathrm{n}$ and $\mathrm{s} / \mathrm{b}$ ratios when imaging through a 
thick optical window. This indicates that the photon gain by the larger NA is counteracted by the spherical aberration, which spreads the focus over a large distance along the optical axis, hence degrades the $\mathrm{s} / \mathrm{n}$ and $\mathrm{s} / \mathrm{b}$ ratios. Another drawback of the Nikon objective is that the original magnification of $100 \times$ has to be reduced to $56 \times$ to resolve single molecules by concentrating photons to fewer pixels on the camera. This also shrinks the FOV by about four times, which limits the throughput of the experiment.

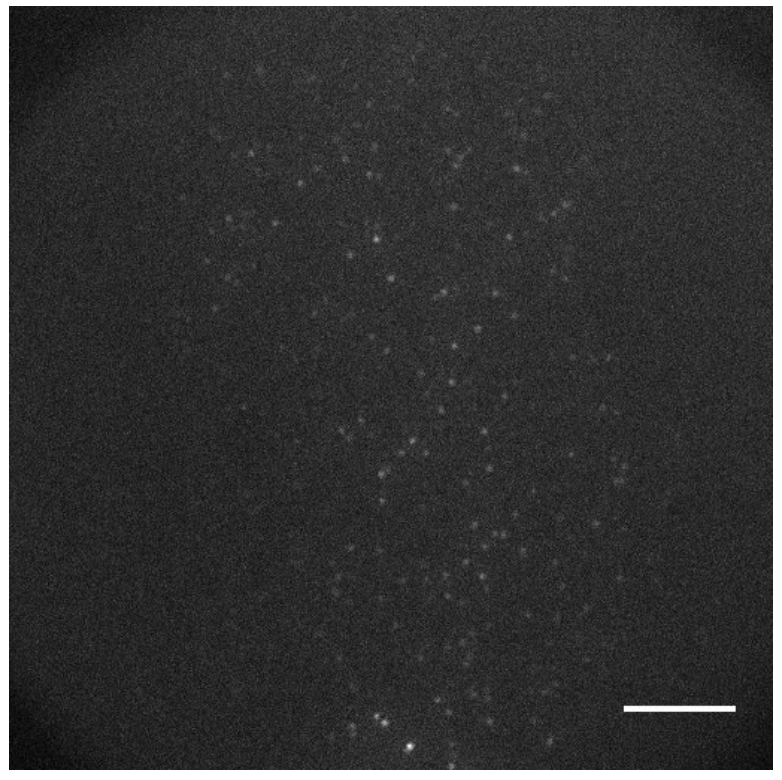

(a) Olympus 0.7 NA

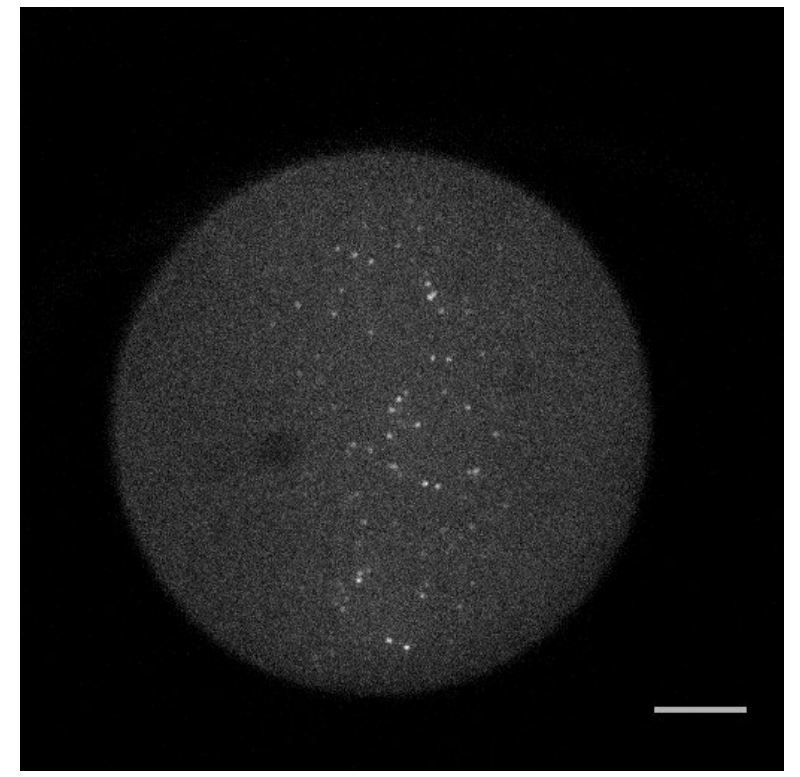

(b) Nikon $0.9 \mathrm{NA}$

Figure 9: Objective comparison for imaging quality. (a) Single Atto647N molecules (ATTO-TEC $\mathrm{GmbH}$, Germany) were imaged through a $0.5 \mathrm{~mm}$ thick quartz window using an Olympus objective with a correction collar (LUCPLFLN $60 \times / 0.7 \mathrm{NA}, \mathrm{WD}=2.2 \mathrm{~mm}$ ). (b) Image taken in the same sample using a Nikon objective without correction collar (TU Plan Apo 100×/0.9 NA, WD = $2.0 \mathrm{~mm}$, magnification adjusted to $\sim 56 \times)$. Images were displayed with the same gray scale. Scale bar: $20 \mu \mathrm{m}$.

In conclusion, when imaging through a thick optical window, the correction collar plays an important role to achieve high imaging quality. Therefore, for the cryostat in this project, the Olympus objective with the correction collar is the appropriate choice. But for cryostats with integrated objective, where no coverslip or optical window is located between the objective and the sample, $0.9 \mathrm{NA}$ objectives could be better due to their higher photon collection efficiency. 


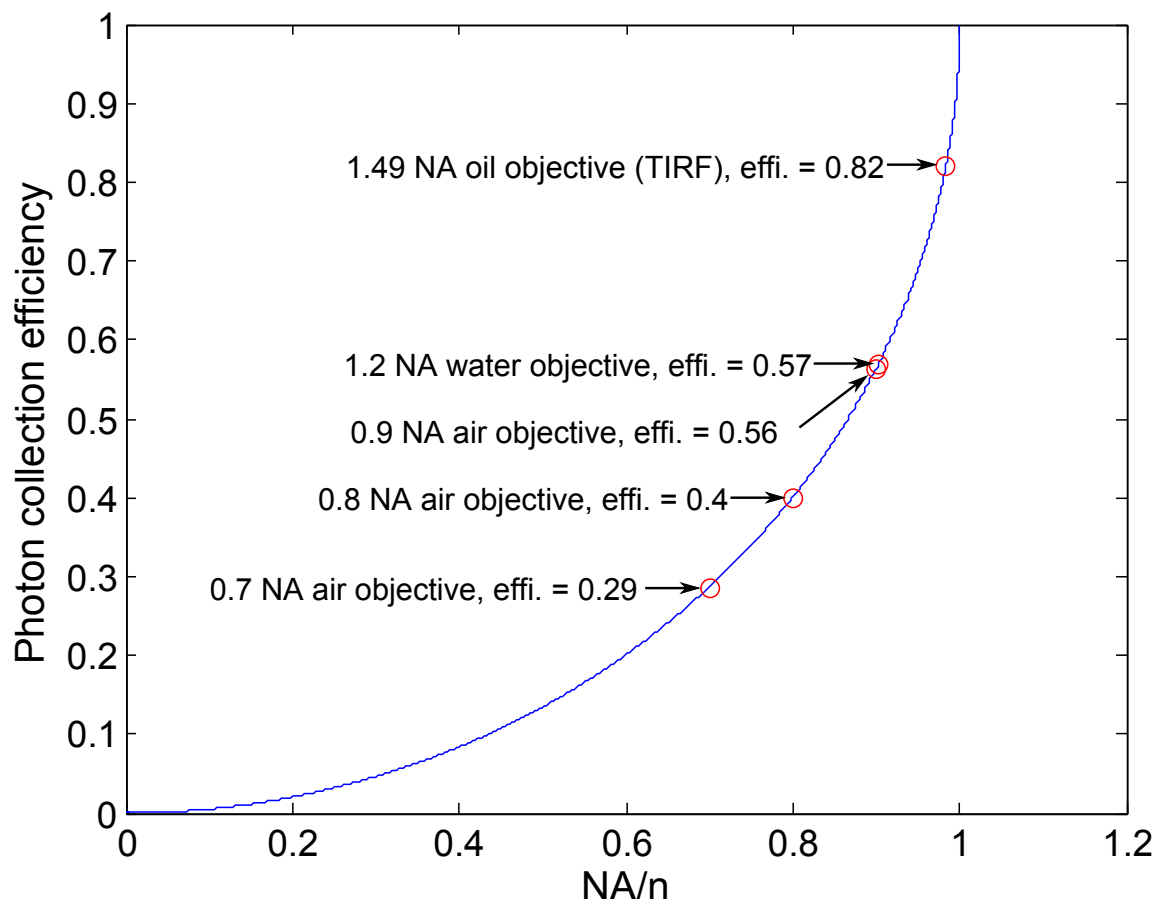

Figure 10: Objective photon collection efficiency with respect to NA. Equation 1 is normalized and plotted with respect to $N A / n$. Commonly used objectives are shown in the plot for comparison.

\subsection{Background minimization}

\subsubsection{Introduction}

One core challenge of single molecule fluorescence microscopy (sm-FM) is to detect the weak fluorescence emitted from single fluorophores. The key to resolve single fluorophores is to increase the $\mathrm{s} / \mathrm{n}$ and $\mathrm{s} / \mathrm{b}$ ratios of the imaging system. This has been realized by the development of various measurement devices and techniques. For example, in confocal microscopy the $\mathrm{s} / \mathrm{n}$ ratio is improved by using sensitive photon detectors such as the photomultiplier tube (PMT) or the single photon avalanche detector (SPAD), which are able to register single photons emitted from the fluorophore. Furthermore, a confocal microscope efficiently suppresses out-of-focus background by placing a pinhole in the conjugated image plane in the detection path, thus providing a high $\mathrm{s} / \mathrm{b}$ ratio.

In wide-field microscopy, traditional CCD/CMOS cameras are typically not sensitive enough to detect single fluorophores at high speed due to the high read-out noise of this devices. This motivated the invention of EMCCD cameras, which amplify the photon signal before read-out. These cameras are capable of detecting single fluorescent molecules with short 
exposure times in the millisecond range with sufficient $\mathrm{s} / \mathrm{n}$ ratio. To increase the $\mathrm{s} / \mathrm{b}$ ratio, various illumination strategies have been developed to suppress the background. These techniques include total internal reflection fluorescence microscopy (TIRF), highly inclined and laminated optical sheet microscopy (HILO), light sheet fluorescence microscopy (LSFM), etc.

In this project, a $0.7 \mathrm{NA}$ air objective with a long working distance of $\sim 2 \mathrm{~mm}$ is used in wide-field configuration. This objective has only about $30 \%$ photon collection efficiency as compared to high NA immersion objectives (Fig.10), thus delivers a moderate s/n ratio. Moreover, the above-mentioned illumination techniques for background-suppression can not be implemented when using the air objective. Therefore, to achieve a high s/b ratio, minimizing the optical background of the imaging system becomes crucial to resolve single molecules. In this section, various background sources in the cryo-imaging system are identified, and the corresponding suppression methods are discussed.

\subsubsection{Background from coverslip}

Normal coverslip glass contains many additional chemical components (e.g. $\mathrm{B}_{2} \mathrm{O}_{3}, \mathrm{Al}_{2} \mathrm{O}_{3}$, $\mathrm{Na}_{2} \mathrm{O}$, etc.) beside silicon dioxide $\left(\mathrm{SiO}_{2}\right)$. These extra ingredients are intentionally added to ease the fabrication (e.g. lowering the melting point of glass), or to introduce certain surface properties (e.g. high wettability, bio-compatibility). However, these components are often weakly fluorescent or luminescent. When imaging under wide-field illumination using a 0.7 NA objective, the fluorescence/luminescence created by the coverslip easily outshines the signal of single fluorescent molecules in the sample.

As demonstrated in Fig. 11.(a), single Atto647N molecules were prepared on a normal coverslip that was thoroughly cleaned using the protocol in appendix 10.5.5. The sample was imaged using a 0.7 NA objective under wide-field illumination. As shown in the image, single molecules could not be resolved due to the strong auto-fluorescence created by the coverslip. To eliminate the coverslip auto-fluorescence, many protocols have been developed, for example high temperature baking or UV bleaching. However, none of these methods is sufficient to decrease the background to a level suitable for single molecule imaging using low NA objectives in wide-field imaging.

To circumvents this problem, fused silica coverslips were used in this project. Fused silica is a $99.99 \%$ pure material consisting of only noncrystalline $\mathrm{SiO}_{2}$. Thus, it creates nearly zero auto-fluorescence regardless of the illumination scheme. Using these coverslips, single 
molecules can be easily resolved with significant s/b ratio (Fig. 11 b).

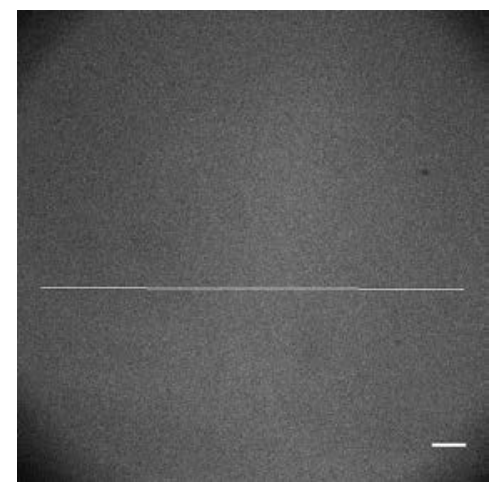

(a)

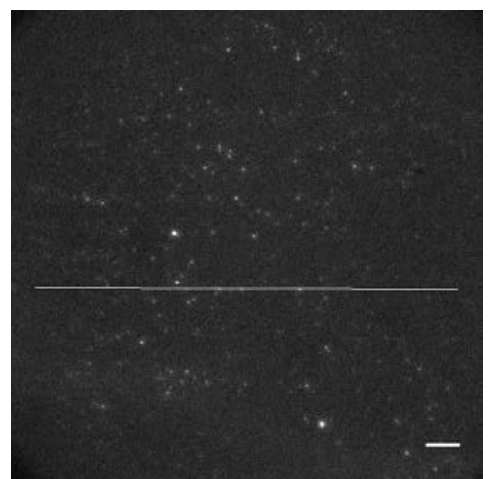

(b)

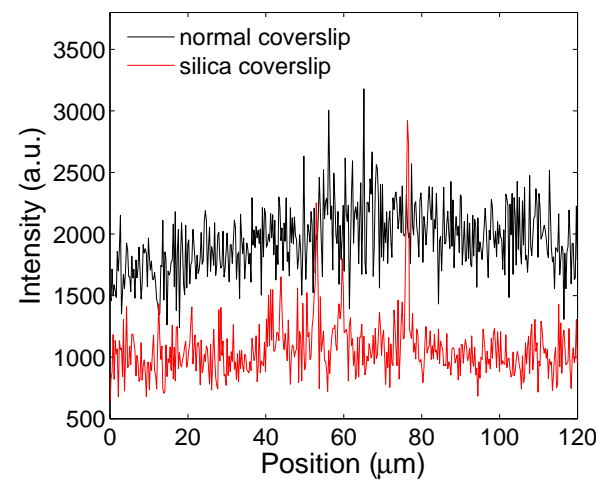

(c)

Figure 11: Background comparison between normal coverslip and fused silica coverslip. (a) A thoroughly cleaned normal coverslip (Menzel Gläser, Germany, cleaning protocol in appendix 10.5.5 covered with single Atto647N molecules was imaged with $637 \mathrm{~nm}$ excitation, $0.1 \mathrm{~s}$ exposure time, and 100 EM-gain. Single molecules were not visible due to the high background created by the auto-fluorescence of the coverslip. (b) A fused silica coverslip (amcoss $\mathrm{GmbH}$ ) cleaned by flaming (Fig.12 was imaged under the same conditions, clearly displaying single Atto647N molecules on a near zero background. (c) Comparison of the intensity profile along lines in (a) and (b). Normal coverslips show about twice as much background as compared to silica coverslips. Scale bar: $10 \mu \mathrm{m}$.

Another advantage of using silica coverslips is that they are very easy to clean. In single molecule experiments, the coverslip surface needs to be thoroughly cleaned to be free of any luminescent contamination. For normal coverslips, the cleaning procedure (appendix 10.5.5) typically involves sonication in organic solvent and strong alkaline solution, followed by plasma cleaning, which is expensive and time-consuming. This wet cleaning method is also not $100 \%$ effective, especially for fluorescent impurities in the blue and green spectral range. In contrast, silica coverslips can be easily cleaned within a minute by flaming the surface using a Bunsen burner. Thanks to the high melting point of the pure silica $\left(\sim 2000^{\circ} \mathrm{C}\right)$, the flame of the Bunsen burner $\left(\sim 1200^{\circ} \mathrm{C}\right)$ removes all (organic) fluorescent impurities on the silica coverslip without melting it. A very simple four-step cleaning procedure is demonstrated in Fig. 12. (Note: This flaming method cannot be applied to normal coverslips because their melting point is lower than the flame temperature of the Bunsen burner.)

One concern for using silica coverslips is their high expense $(\sim 10$ euros/piece). However, the used coverslips can be cleaned and recycled, which makes them affordable for experiments in the daily routine. To clean lightly used silica coverslips containing mainly organic samples and minimal salts, the procedure in Fig.12 can be directly applied. For heavily used coverslips containing many salts or metals, they can be pre-washed using conventional protocols 


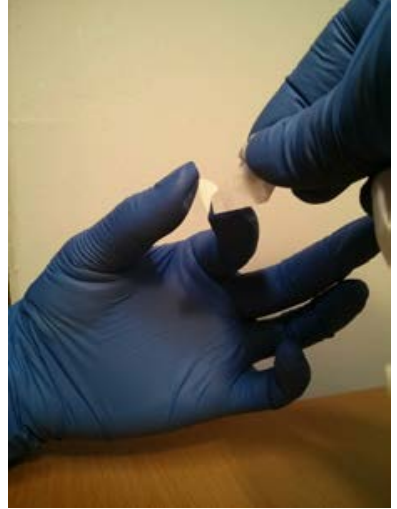

(a)

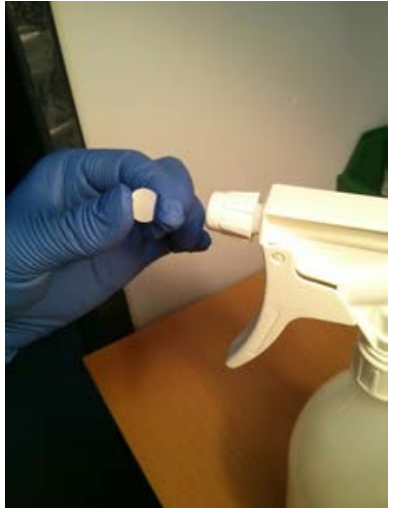

(b)

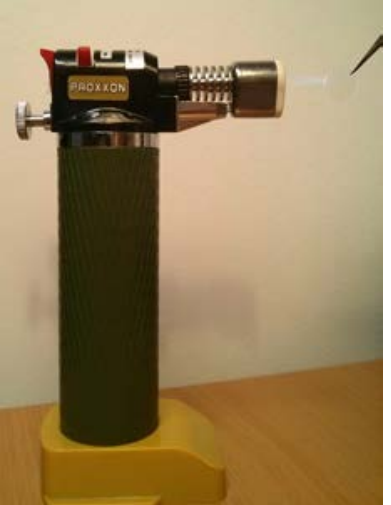

(c)

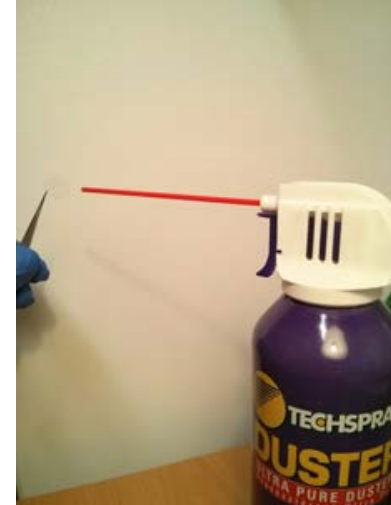

(d)

Figure 12: Cleaning procedure for silica coverslip. (a) Wipe the coverslip surface with $70 \%$ Ethanol using a lab tissue. (b) Rinse the coverslip thoroughly with a high pressure spray of $70 \%$ Ethanol. (c) Scan the coverslip surface with the outer flame of a Bunsen burner. (d) Cool down the coverslip using a lab duster.

(e.g. appendix 10.5.5), and then be flamed. Plasma cleaning should be avoided for silica coverslips, because it often introduces contaminates as observed in practice (Fig. 13).

All in all, silica coverslips are optimal substrates for sample preparation. They do not only provide zero background for single molecule imaging, but are also very easy to clean. The possibility to recycle them makes it also affordable to use them in the daily routine.

\subsubsection{Background from sample holder}

The sample holder is made of oxygen-free copper (Fig. 14a), which creates a strong fluorescence and luminescence background under laser illumination, as shown in Fig.14(e). The polished metal surface also heavily reflects the excitation light, which further increases the background. Therefore, the sample holder cannot be directly used as a substrate to mount the silica coverslip for single molecule imaging.

To solve this problem, a thin opaque metal disc (Fig.14 b) was made to cover the sample holder surface (Fig. 14 c), and the coverslip was mounted on top of it (Fig. 14d). This $0.1 \mathrm{~mm}$ thick opaque disc was made by quickly flaming a piece of stainless steel using the Bunsen burner. After flaming, stainless steel becomes non-fluorescent and non-reflective, and delivers almost zero background (Fig. $14 \mathrm{f}$ ). This opaque disc is a crucial component that enables single molecule imaging with high s/b ratio, and was used for all experiments in this thesis. 

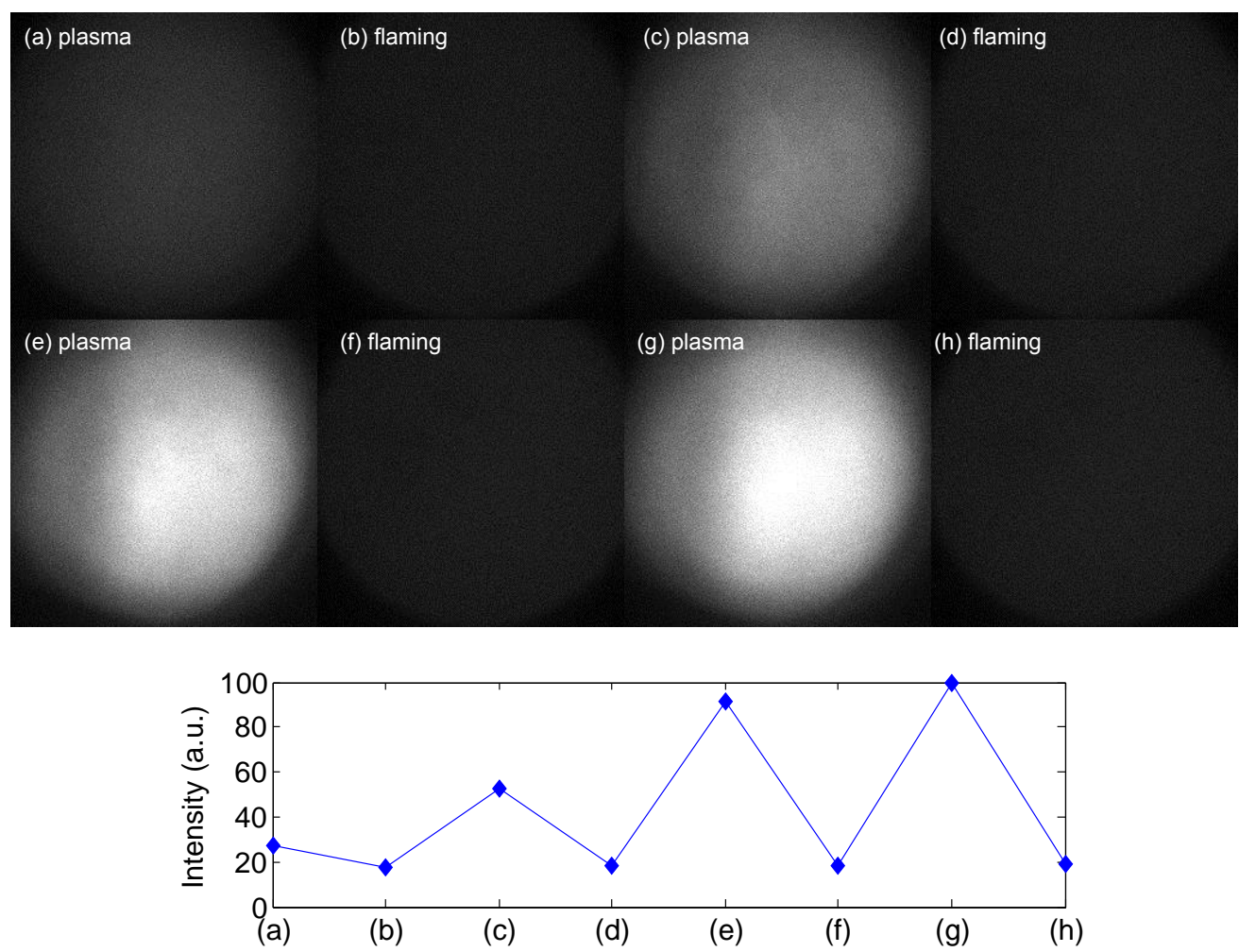

Figure 13: Silica coverslip contamination by plasma cleaning. Clean silica coverslip was treated with 15 min plasma cleaning and Bunsen burner flaming alternatingly. The coverslip was excited at $637 \mathrm{~nm}$, and imaged through a 685-50 emission filter. Images showed a growing background after each plasma cleaning. This background vanished each time after flaming with the Bunsen burner.

A potential drawback of using the opaque disc is that it absorbs all excitation light, and thus will be locally heated. This local heating brings some negative side-effects. For example, as the opaque disc is only loosely clamped on the sample holder by a magnetic ring (Fig. $14 \mathrm{~d}$ ), the heating-induced thermal expansion/shift of the disc will be directly transmitted to the sample, causing additional sample drift. Because the total sample drift observed in practice is moderate, and can be post-corrected, no further optimization has been carried out on this point. If the thermal-induced sample drift becomes intolerable in potential later applications, the drift can be reduced by e.g. soldering the opaque disc to the sample holder, or directly processing the OFC surface to be opaque.

Another related concern is that the sample might also be heated up locally by the opaque disc. It is difficult to examine the exact temperature in the illuminated area on the coverslip. But the local heating should be rather minor, because the thermal sensor does not register any global temperature rise upon laser irradiation, and significant improvement in fluorophore photostability is observed upon cooling (section 6.1). The high transparency of the silica coverslip should minimize direct heating of the coverslip by the laser, and the high thermal 
conductivity of the cooling units should deliver effective dissipation of the laser energy.

In short, the opaque disc removed the strong background of the sample holder, and enabled single molecule imaging with high $\mathrm{s} / \mathrm{b}$ ratio.

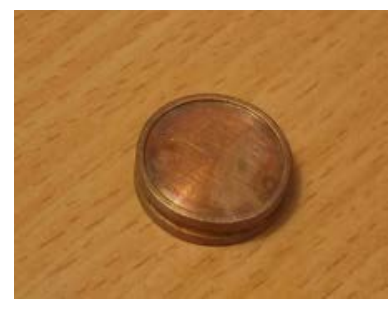

(a)

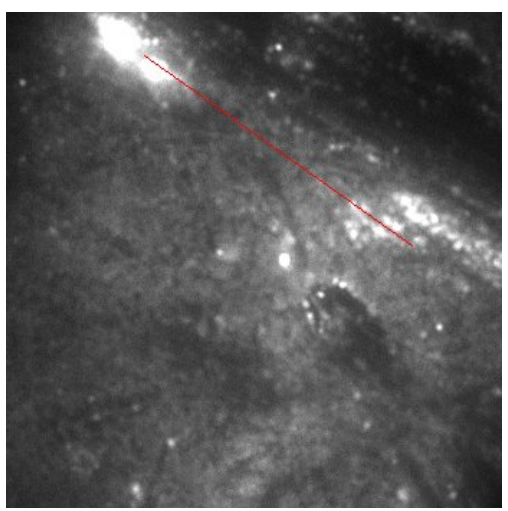

(e)

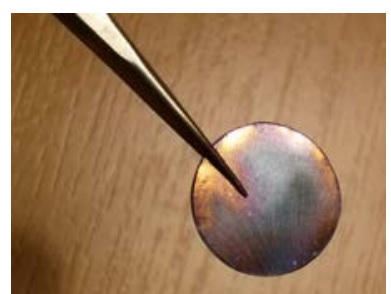

(b)

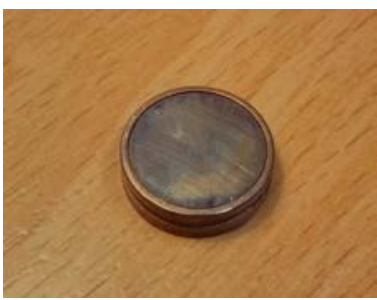

(c)

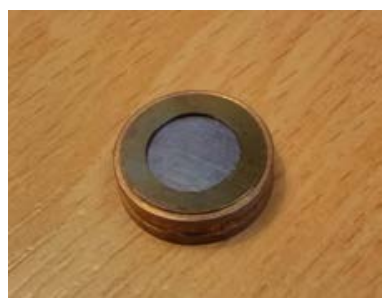

(d)

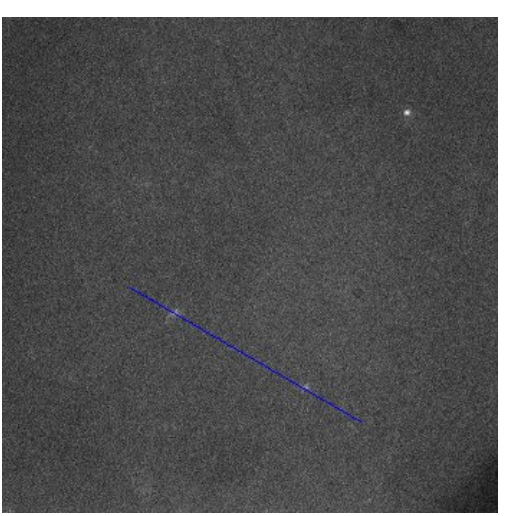

(f)

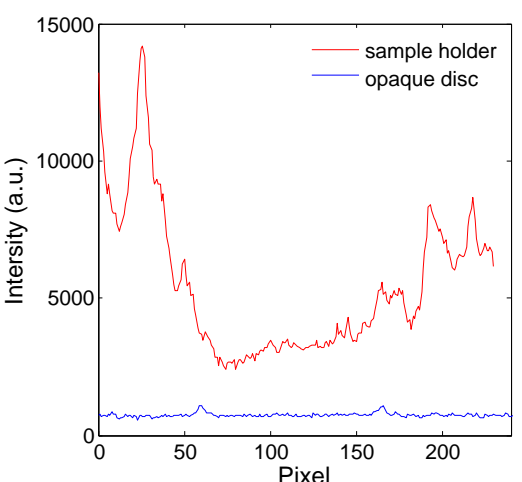

$(\mathrm{g})$

Figure 14: Opaque disc for removing sample holder background. (a) Sample holder made of oxygen-free copper. (b) Custom-made non-reflective, non-fluorescent disc. (c) Sample holder surface covered by the opaque disc. (d) Opaque disc sandwiched between the sample holder and the coverslip, held together magnetically by a clamping ring. (e) Fluorescence image of the sample holder surface in (a), taken with $637 \mathrm{~nm}$ excitation, 685-50 emission filter, and 0.2 s exposure time. (f) Fluorescence image of the opaque disc surface in (b), taken under the same conditions. (g) Comparison of the intensity profile along the lines in (e) and (f).

\subsubsection{Background from objective}

Modern objectives contain many lenses made of glass, which is weakly fluorescent. Some of these lenses are glued together using optical adhesive, which is often fluorescent as well. Moreover, each surface of a lens reflects a certain amount of the excitation laser despite anti-reflection coatings. The reflected laser together with the auto-fluorescence created by the bulky lens array form a strong background source when laser light is passed though the objective. However, the objective-generated background is rarely discussed in the fluo- 
rescence microscopy literature, because it usually contributes a rather minor signal to the overall background for most of the microscopy techniques using high NA objectives. For example, in confocal microscopy the background light generated within the objective will be completely blocked by the imaging pinhole, because the objective lenses are located far away from the focal plane. In TIRF/HILO microscopy, the objective lenses are illuminated by the excitation laser only on the side far away from the optical axis, which contributes minimally to the image background. However, in this project, when using a 0.7 NA air objective in normal wide-field illumination scheme, the objective-introduced background starts to limit the $\mathrm{s} / \mathrm{b}$ ratio for single molecule imaging.

As demonstrated in Fig. 15, under $637 \mathrm{~nm}$ laser illumination, placing an air or a water objective into the microscope boosted the image background by a factor of $\sim 2.1$ or $\sim 2.7$, respectively. When placing a silica coverslip at the focus of the water objective, another $\sim 7 \%$ was added to the background.

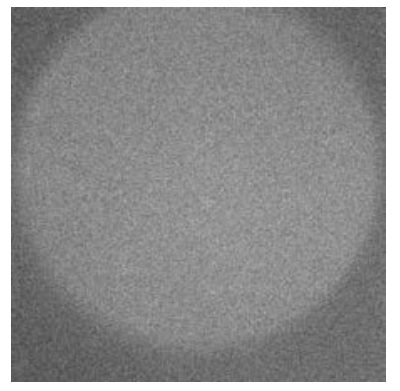

(a) No objective, Int $=34$

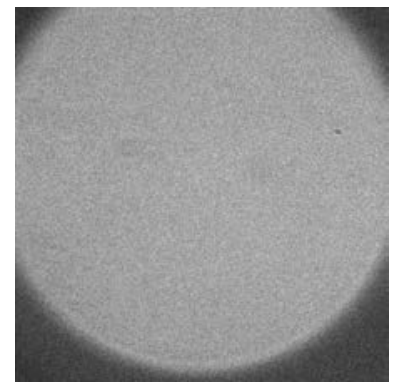

(b)
Air objective, (c) Int $=72$

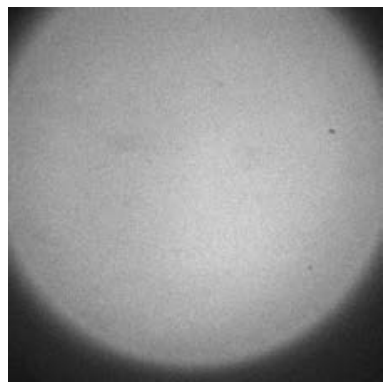

(c) Water objective, Int $=93$

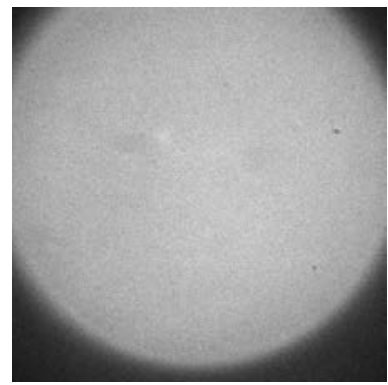

(d) Water objective + silica coverslip, Int $=100$

Figure 15: Objective-introduced background. Images were captured on an EMCCD camera with $637 \mathrm{~nm}$ illumination, 685-50 emission filter, 1s exposure time, and zero EM-gain in four situations: (a) No objective was present in the microscope. (b) An air objective (LUCPLFLN, Olympus, $60 \times / 0.7 \mathrm{NA}$ ) was placed in the microscope. (c) A water objective (UPlanSApo, Olympus, $60 \times / 1.2 \mathrm{NA}$ ) was placed in the microscope. (d) A clean silica coverslip was placed at the focus of the water objective. Average intensities of the illuminated area were calculated and normalized with respect to (d). Images were displayed with the same gray scale.

The spectrum of the background signal (Fig. 16) revealed components of the excitation laser as well as fluorescence from the detection window. This indicates that the background is created by the reflected laser on the multiple surfaces of the objective lenses and the coverslip, as well as by the auto-fluorescence of the lenses. This agrees with the fact that the water objective contains more lenses than the air objective, thus creating a stronger background.

As the lenses inside the objective are far from the focal plane, iris filtering in the image plane 
has been attempted to reduce the background. To create a new image plane for filtering, a pair of achromatic lenses were placed before the camera tube lens to form a $4 \mathrm{f}$ scheme. At the focal plane (image plane) between these two lenses, an iris was placed to reject the outof-focus components of the image, in analogy to the pinhole filtering in confocal microscopy. However, no evident background reduction was observed till the iris was half closed (Fig. 17). This indicates that iris filtering on the image plane is hardly effective to remove the out-offocus components when using a low NA objective with long working distance.

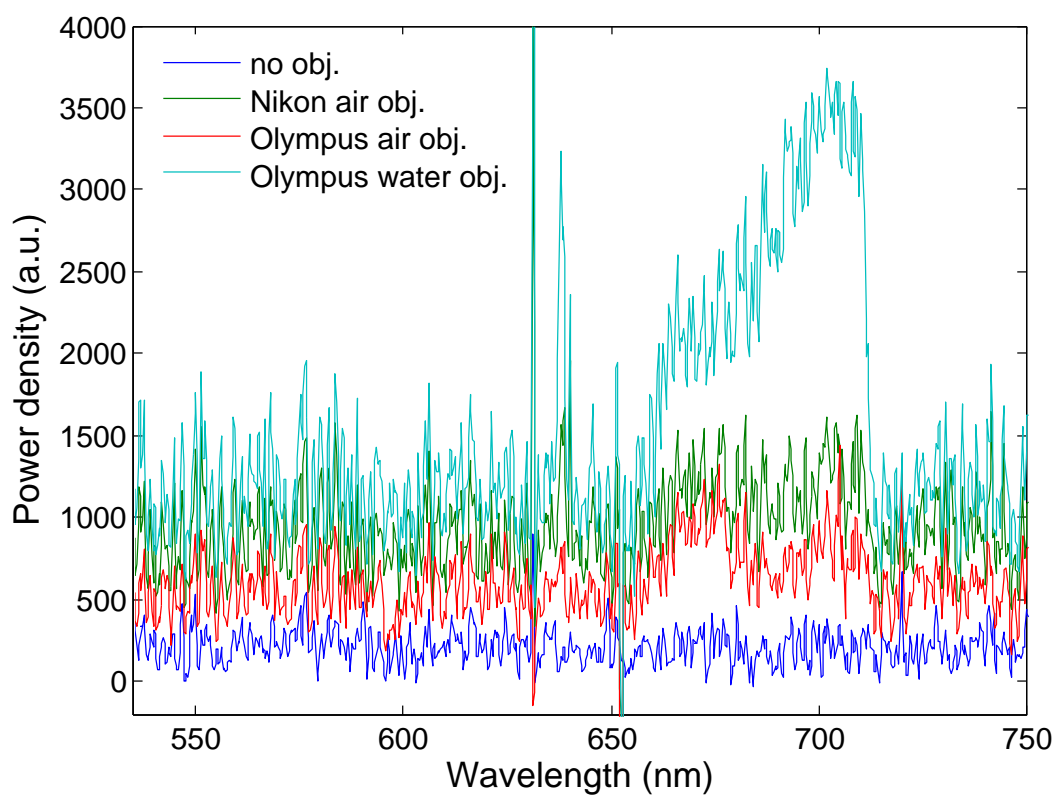

Figure 16: Spectrum of objective-introduced background. Objectives were placed in the microscope, and spectra were measured under $637 \mathrm{~nm}$ laser illumination through a 685-50 emission filter, using a CCD array spectrometer (AvaSpec-2048-USB2, Avantes) with 30 s exposure time, averaged for 6 measurements. Tested objectives were: Nikon air objective (TUPlanApo, 100×/0.9 NA), Olympus air objective (LUCPLFLN, $60 \times / 0.7 \mathrm{NA}$ ), and Olympus water objective (UPlanSApo, $60 \times / 1.2 \mathrm{NA})$.

Another way of eliminating the background is using time-gated imaging, because the laser reflection and the auto-fluorescence of the glass occur on different time scales as compared to the fluorescence of dyes. However, this technique requires fast cameras that can be triggered on the picosecond time scale, which are not yet available. But photodetector manufactures report rapid progresses with the development of these fast cameras, which will become available in the near future. To the date of this project, the objective-introduced background is the limiting factor for a further increase of the s/b ratio when using a $0.7 \mathrm{NA}$ objective with normal wide-field illumination. 


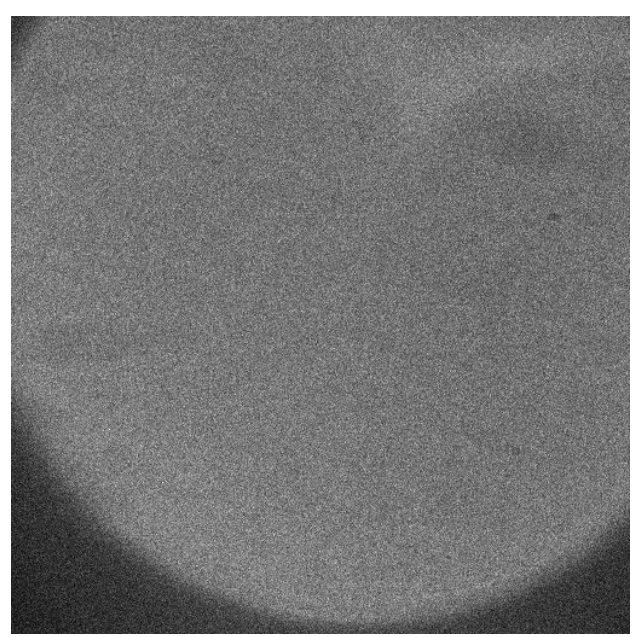

(a) Iris open, Int $=6326$ (a.u.)

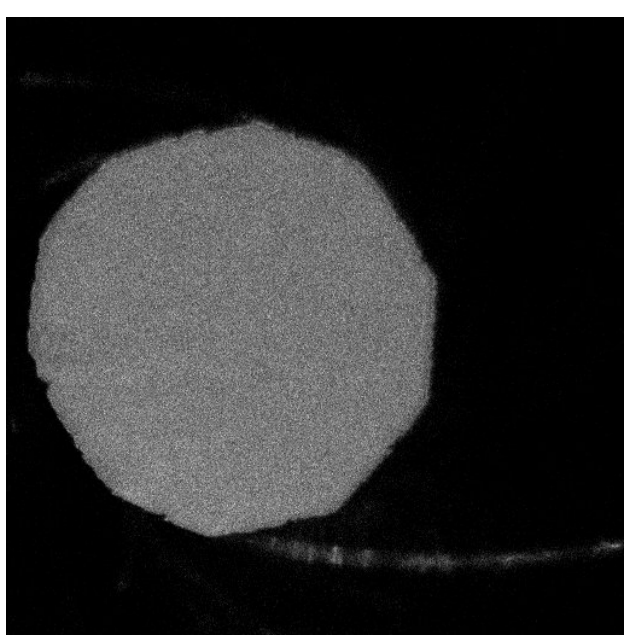

(b) Iris half closed, Int $=6307$ (a.u.)

Figure 17: Iris filtering at the image plane. A $4 \mathrm{f}$ system was introduced in front of the camera tube lens using a pair of achromatic lenses ( $\mathrm{f}=75 \mathrm{~mm}$, AC508-075-A-ML, Thorlabs, GmbH, Germany). An iris was placed at the newly created image plane between the two lenses to remove the out-offocus components of the image. Average background intensities were measured when the iris was fully opened (a) and half closed (b). Images were taken with $637 \mathrm{~nm}$ excitation, 100 EM-gain, and $0.2 \mathrm{~s}$ exposure time.

\subsection{Imaging quality}

The optimizations of the imaging system by using aberration-correcting objectives, backgroundfree silica coverslips, and a background-removing opaque disc have resulted in successful single molecule imaging in the cryostat at $89 \mathrm{~K}$.

As demonstrated in Fig. 18, single Atto647N molecules were spin-coated on a silica coverslip, and the sample was imaged at $89 \mathrm{~K}$ using the Olympus air objective with an appropriate setting of the correction collar. Single molecules were resolved with excellent $\mathrm{s} / \mathrm{n}$ and $\mathrm{s} / \mathrm{b}$ ratios, and the first-order ring of the diffraction pattern was clearly visible (Fig. $18 \mathrm{a}-\mathrm{c}$ ). More interestingly, after defocusing $\sim 3 \mu \mathrm{m}$, the defocused patterns were also resolved (Fig. $18 \mathrm{~d}$ f). These patterns had identical shapes across the FOV, and had rotationally symmetric intensity profiles. This indicates superb imaging quality with minimal field distortion and spherical aberration.

It was noticed that the imaging quality was extremely sensitive to the setting of the correction collar. Therefore, a complete elimination of the spherical aberration is in practice hardly achievable by manually adjusting the collar. As a result, the detected PSF often appears slightly larger than the theoretically predicted size. Nevertheless, this effect can be treated 
as a systematic error, and can be corrected in the post-analysis. The slight broadening of the PSF also does not affect any localization based analysis, because it does not change the centroid position of the PSF.
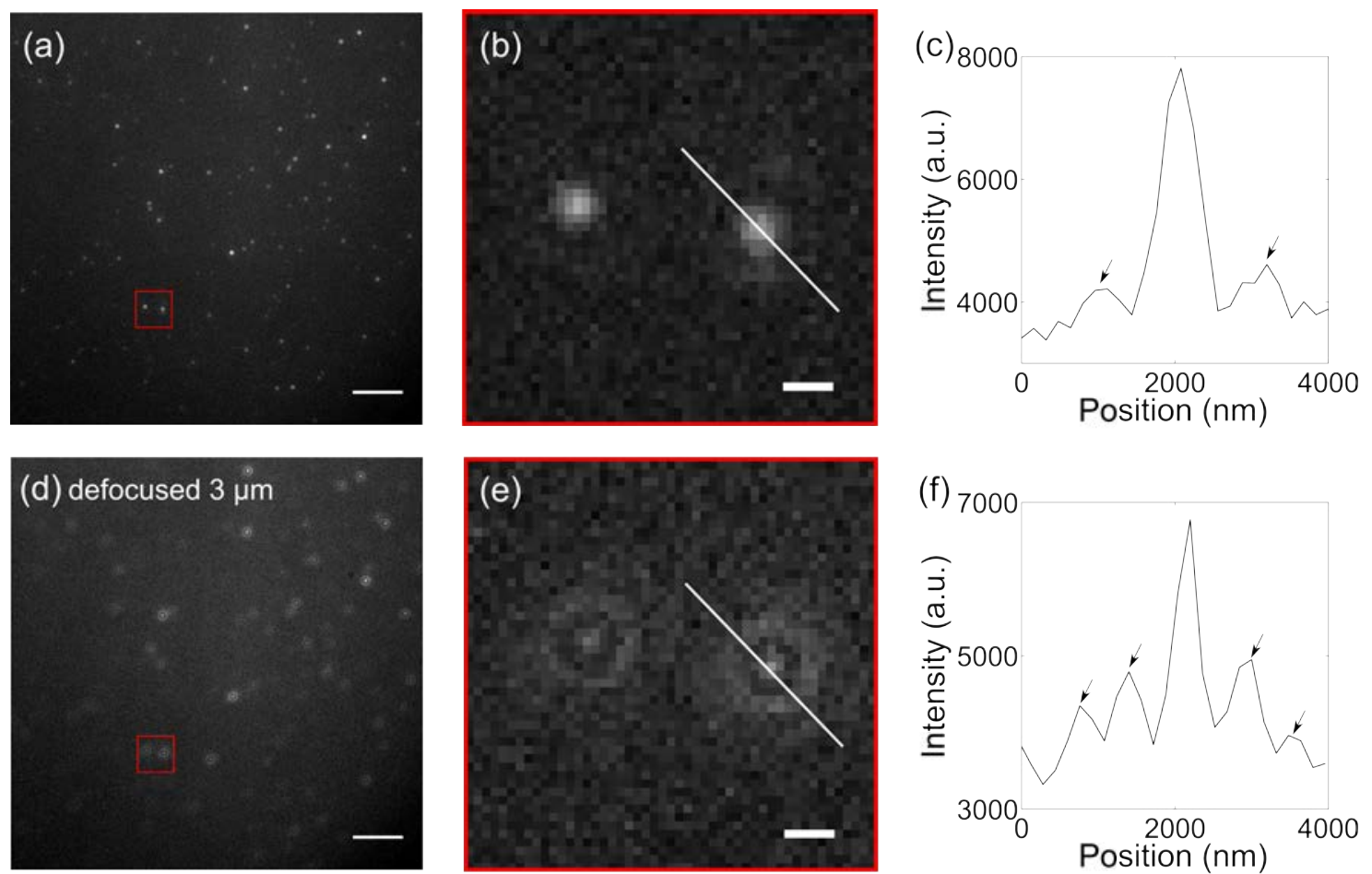

Figure 18: Single molecule imaging at $89 \mathrm{~K}$. Single Atto647N molecules were imaged on a silica coverslip at $89 \mathrm{~K}$ with $1 \mathrm{~s}$ exposure time and 100 EM-gain. The original tube lens $(\mathrm{f}=180 \mathrm{~mm})$ was replaced by an achromatic doublet $(\mathrm{f}=300 \mathrm{~mm})$ to deliver an enlarged magnification of $100 \times$. (a) The sample was imaged in focus. (b) Zoom-in on the red square in (a). (c) Intensity profile along the line in (b). (d) The sample was imaged after defocused $\sim 3 \mu \mathrm{m}$. (e) Zoom-in on the red square in (d). (f) Intensity profile along the line in (e). Arrows pointed to the side rings of the diffraction pattern.

Remarkably, when imaging single molecules with fixed dipole orientations (e.g. when frozen), a low NA air objective is advantageous over a high NA immersion objective for single molecule localization. The reason is that a low NA objective is insensitive to the orientation of the emitter's dipole ${ }^{[68}$, thus always delivers symmetric PSFs regardless of the molecule orientation. This greatly simplifies the localization analysis based on PSF fitting, because one can always assume that the PSF has a 2D Gaussian shape. Moreover, a low NA objective is less sensitive to the vertical drift due to the larger focal depth, which is especially beneficial for long-term measurements. 


\section{Sample Preparation}

\subsection{Introduction}

Preparing samples for cryogenic imaging is a task that requires great know-how and experience. A dedicated workflow has been developed for sample preparation in cryo-EM. The involved techniques include sample vitrification in amorphous ice using high pressure freezing [58] or plunge freezing [57], sample segmentation using automated diamond knife, sample fixation on the suspended carbon film on the EM grid, etc. Although these techniques can be used to prepare samples for cryo-FM as well, the expensive apparatus and the required expertise make them not feasible for most of the labs.

If only cryo-FM is desired (without correlative measurement with cryo-EM), samples can also be prepared on coverslips using conventional protocols. Special care has to be given only to the final mounting medium, which should have glassy freezing character and vacuum compatibility. The choice of the mounting medium strongly depends on the sample properties. In this section, three mounting strategies are introduced, which cover most of the sample types.

\subsection{Dry mounting}

For small rigid single molecule samples prepared in solution (e.g. DNA constructs, small proteins, etc.), the solution can be directly deposited onto the coverslip by spin-coating or pipetting, and left for drying in air. These samples are typically rigid enough to keep their structures after being dried, and are also vacuum compatible.

This "dry mounting" is the easiest method to prepare cryo-compatible samples, and it has several advantages. (i) The absence of the mounting medium assures a minimal background, hence delivers a high s/b ratio. (ii) The dry sample can be conveniently loaded into the cryostat at RT, and then gradually cooled down without concern for water crystallization. (iii) The silica coverslip has high surface smoothness of $1.5 \mathrm{~nm}$ RMS, as shown in atomic force microscopy (AFM) image in Fig.19(a)-(b). After drying, the sample mostly lies "flat" on the surface, as demonstrated with DNA rods in Fig. 20. (iv) Dry mounting does not require additional sample immobilization. This avoids any uncontrollable geometrical change of the intermediate immobilization layer such as bovine serum albumin (BSA) after drying. As 
shown in Fig. 19 (c)-(d), a BSA-coated coverslip surface displayed many nonuniform "islands" after drying. All in all, dry mounting provides the maximal convenience and reliability for cryo-sample preparation.

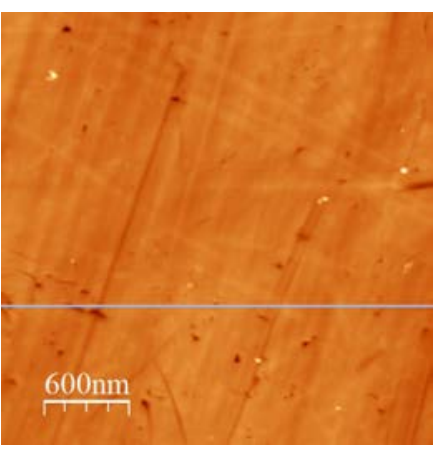

(a)

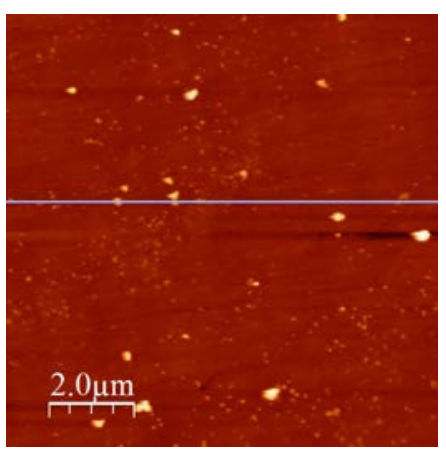

(c)

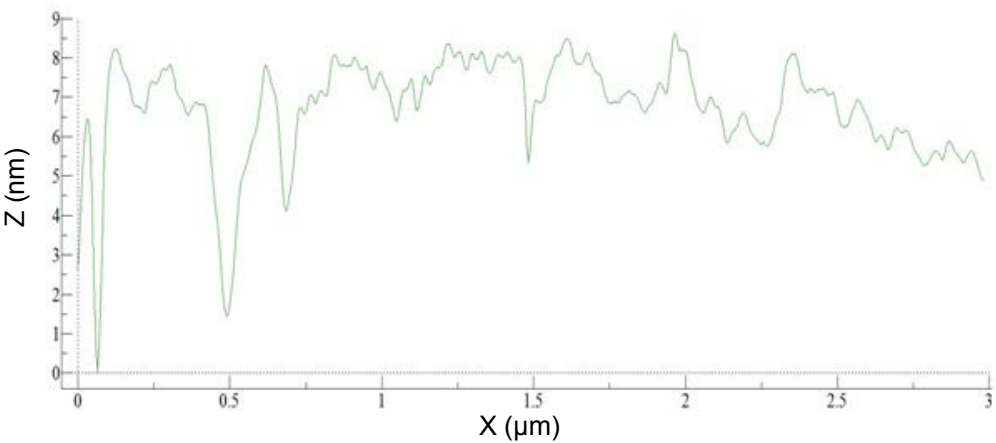

(b)

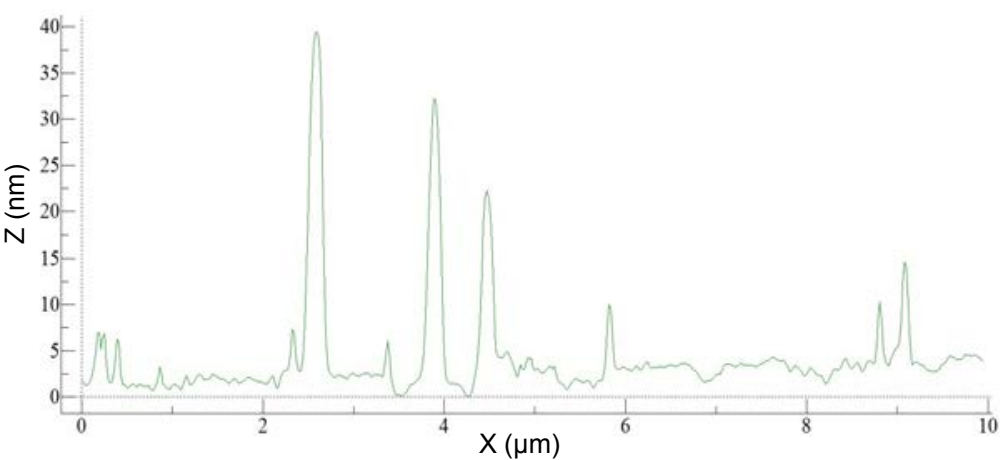

(d)

Figure 19: AFM image of silica coverslip surface. (a) AFM image of the surface of a cleaned silica coverslip. (b) Height profile along the line in (a). (c) AFM image of a silica coverslip surface coated with $0.2 \mathrm{mg} / \mathrm{ml} \mathrm{BSA}$ after air-dried. (d) Height profile along the line in (c). AFM image taken by Iwan Schaap.

\subsection{Polymer medium mounting}

For samples of greater sizes, such as vesicles, large protein complexes, or cellular structures, a mounting medium is typically required to maintain their physical structures. For these samples, two polymer based mounting media - glycerol and polyvinyl alcohol (PVA) - are preferred for several reasons.

First, glycerol has a very low evaporation rate $\underline{69}$, and PVA behaves as a solid medium after being dried, thus they both can be used in vacuum. Secondly, these mounting media exhibit a glassy cooling behavior, thus they can be loaded into the cryostat at RT, and then gradually 


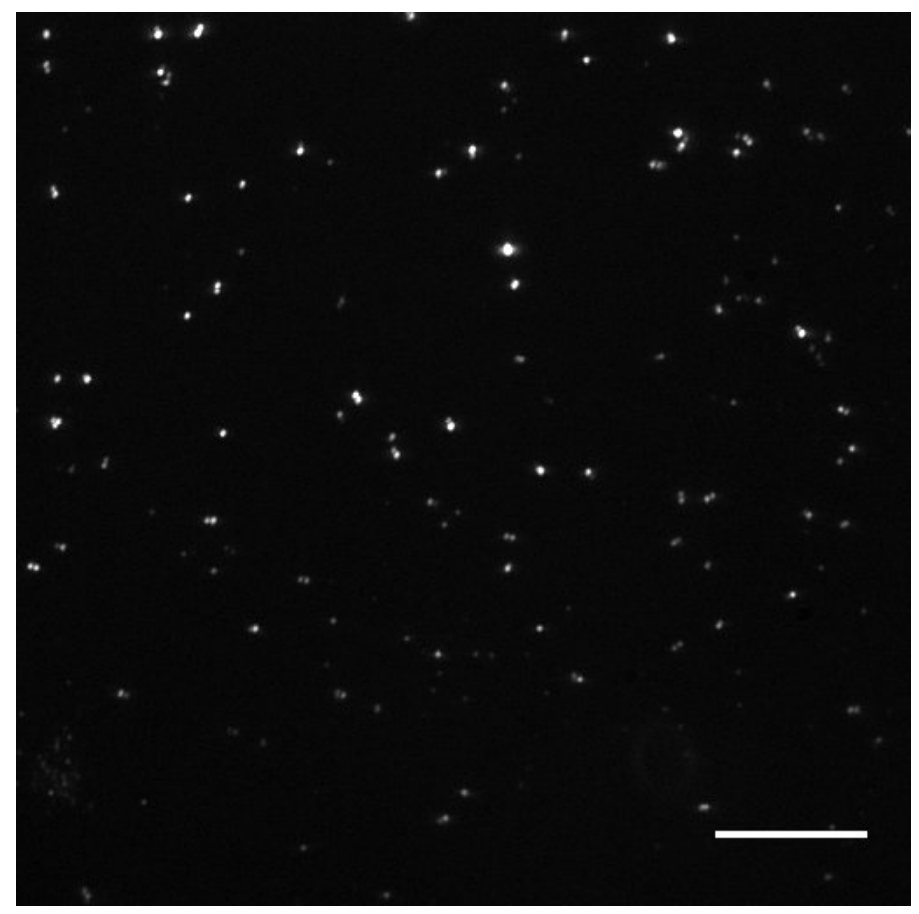

Figure 20: DNA rods on silica coverslip. DNA rods (350R, GATTAquant GmbH, Germany) were marked at two sites separated by $350 \mathrm{~nm}$ using multiple Atto647N dye molecules. The rod solution was deposited onto a silica coverslip using a pipette, and left air-dried. TIRF image using a 1.49 NA objective at $140 \times$ magnification resolved the two marking positions for almost all the rods, indicating that they lie flat on the surface after dried. Scale bar: $10 \mu \mathrm{m}$.

cooled down without crystallization. As demonstrated in Fig.21, after cooling PVA from RT $(293 \mathrm{~K})$ to $89 \mathrm{~K}$ in the cryostat, the background level remained the same, indicating the absence of crystallization. Thirdly, these media deliver minimal background, which makes the observation of single fluorescent molecules possible (Fig.21).

In summary, if a mounting medium is necessarily required, glycerol and PVA are optimal choices due to their vacuum compatibility, glassy cooling profile, and minimal background.

\subsection{Aqueous medium mounting}

Aqueous mounting medium cannot be avoided for certain samples to keep their structural or biological properties, e.g. for cells cultivated in a particular buffer. To prepare these samples for cryo-imaging, rapid freezing becomes necessary to avoid ice crystal formation in the sample. Ice crystals damage the sample in multiple ways. For example, they destroy fine structures of the sample, and deliver strong background for both cryo-EM and cryo-FM. 


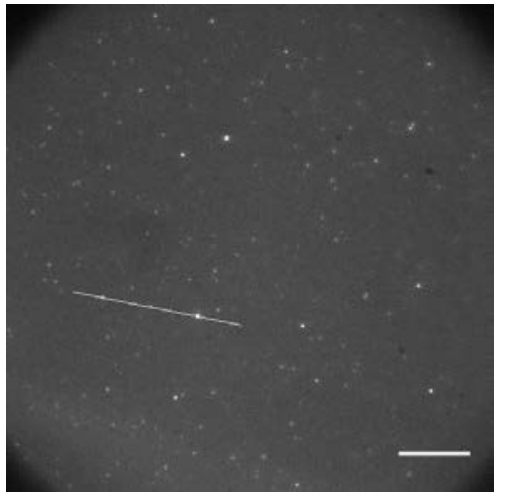

(a) $293 \mathrm{~K}$

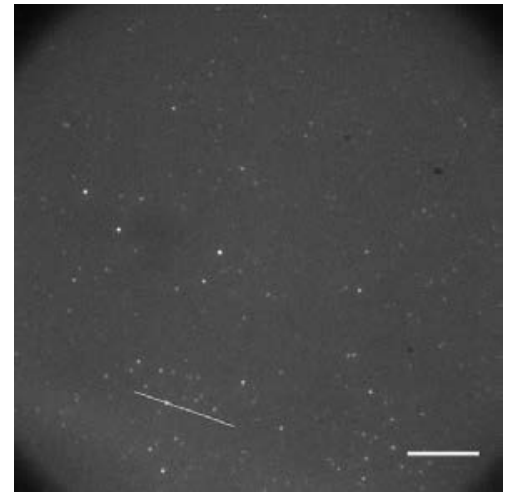

(b) $89 \mathrm{~K}$

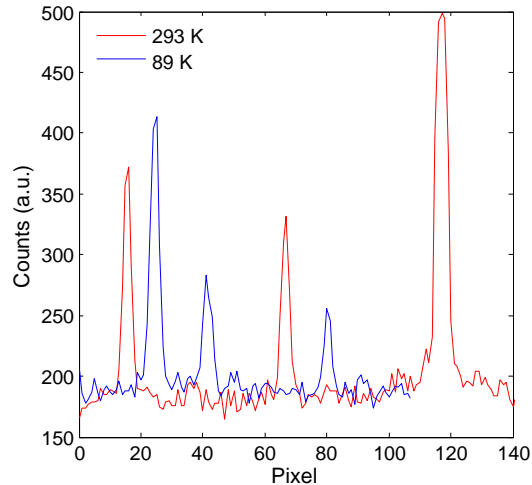

(c) Line profile

Figure 21: PVA as mounting medium. Single Atto647N molecules were dissolved in 1\% PVA in water. $1 \mu \mathrm{L}$ solution was spread on a silica coverslip using a pipette, and left air-dried. The sample was loaded into the cryostat at RT $(293 \mathrm{~K})$, and was gradually cooled down to $89 \mathrm{~K}$. (a) Image taken at $293 \mathrm{~K}$ with $1 \mathrm{~s}$ exposure time and $100 \mathrm{EM}$-gain. (b) Image taken at $89 \mathrm{~K}$ under the same condition. (c) Background comparison between the lines in (a) and (b). Scale bar: $20 \mu \mathrm{m}$.

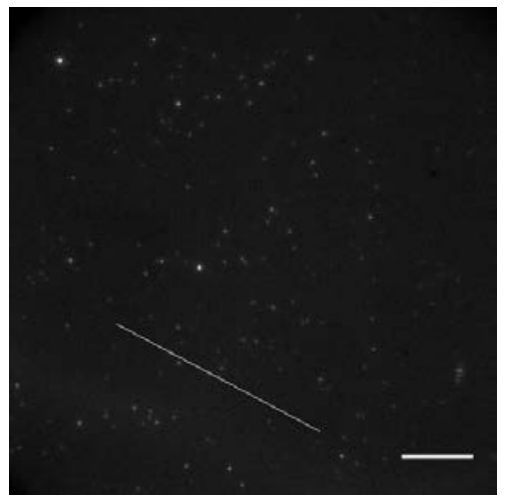

(a) $293 \mathrm{~K}$

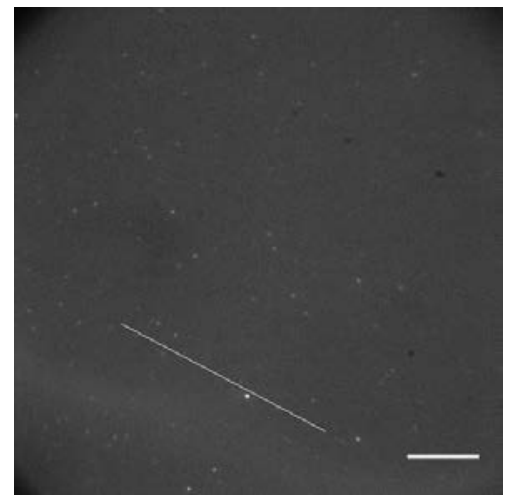

(b) $89 \mathrm{~K}$

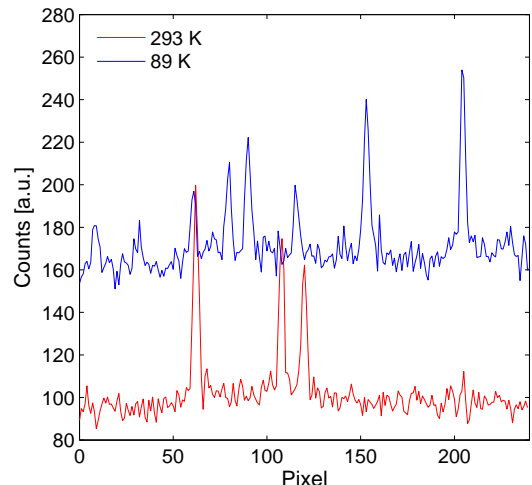

(c) Line profile

Figure 22: Water crystallization by gradual cooling. $0.25 \mu \mathrm{L}$ solution of Atto647N molecules was sandwiched between two silica coverslips of diameter $12 \mathrm{~mm}$, delivering an aqueous layer of $\sim 2.2 \mu \mathrm{m}$ thick. The sample was loaded into the cryostat at RT $(293 \mathrm{~K})$, and was gradually cooled down to $89 \mathrm{~K}$. (a) Image taken at $293 \mathrm{~K}$ with $1 \mathrm{~s}$ exposure time and $100 \mathrm{EM}$-gain. (b) Image taken at $89 \mathrm{~K}$ under the same condition. (c) Background comparison between the lines in (a) and (b). Scale bar: $20 \mu \mathrm{m}$.

Cooling in the cryostat is not fast enough to avoid water crystallization. As demonstrated in Fig.22, a $2.2 \mu \mathrm{m}$ thin water layer containing single Atto647N molecules was cooled down from RT to $89 \mathrm{~K}$ in the cryostat. The crystallization of water boosted the background level by about 1.6 times, and hindered the observation of single molecules. 
Therefore, samples have to be frozen using special rapid cooling techniques prior to the experiment, and then can be loaded into a pre-cooled cryostat below the glass transition temperature of water $(\sim 135 \mathrm{~K})$. This can be done using a new workflow developed in this project (section 5.2). However, the expensive rapid freezing apparatus and the effortdemanding cryo-transfer make the aqueous medium in general not favored for cryo-sample preparation.

\section{System Operation}

\subsection{Sample transfer at RT}

Samples prepared in a dehydrated form by "dry mounting", or fixed in a medium of glassy freezing profile (e.g. glycerol or PVA) can be loaded in to the cryostat at RT, and then gradually cooled down to CT.

The sample transfer is straight forward. First, the silica coverslip or the EM grid containing the sample is fixed on the sample holder by magnetic clamps (Fig. 3). Then, the "warm" cryostat is opened at RT, and the sample holder is loaded onto the sample mount. Next, the cryostat is closed, and a vacuum is generated using a turbo pump. When the pressure in the cryostat reaches $\sim 10^{-2} \mathrm{~Pa}$ (measured by the integrated barometer in the turbo pump), the vacuum valves are closed, and the pump is stopped. Lastly, the LN2 is fed into the tank, and the sample is gradually cooled down with a temperature profile shown in Fig.7. After ca. $10 \mathrm{~min}$, the sample reaches a stable temperature of $89 \mathrm{~K}$, and is located in a vacuum of $\sim 10^{-3} \mathrm{~Pa}$ (pressure further reduced by the cold tank).

Sample transfer at RT is convenient, and the whole process takes only about 10 minutes to finish. The sample can be almost instantly measured after the injection of LN2 due to the fast cooling rate of the cryostat.

\subsection{Sample transfer at CT}

There are in general three situations in which samples need to be loaded into the cryostat at CT. (i) The sample is prepared in a hydrated mounting medium, and is pre-frozen using fast freezing techniques. (ii) High throughput is desired, i.e. multiple samples need to be examined in a series at CT. (iii) Correlative measurements with cryo-EM are required. 
Sample transfer at CT must avoid any exposure to the humid air to prevent ice condensation, and must be carried out below the glass transition temperature of water $(\sim 135 \mathrm{~K})$ to avoid ice crystal formation in the sample. To meet these requirements, a workstation was constructed, and a workflow was developed for intact sample transfer at CT (Fig.23).
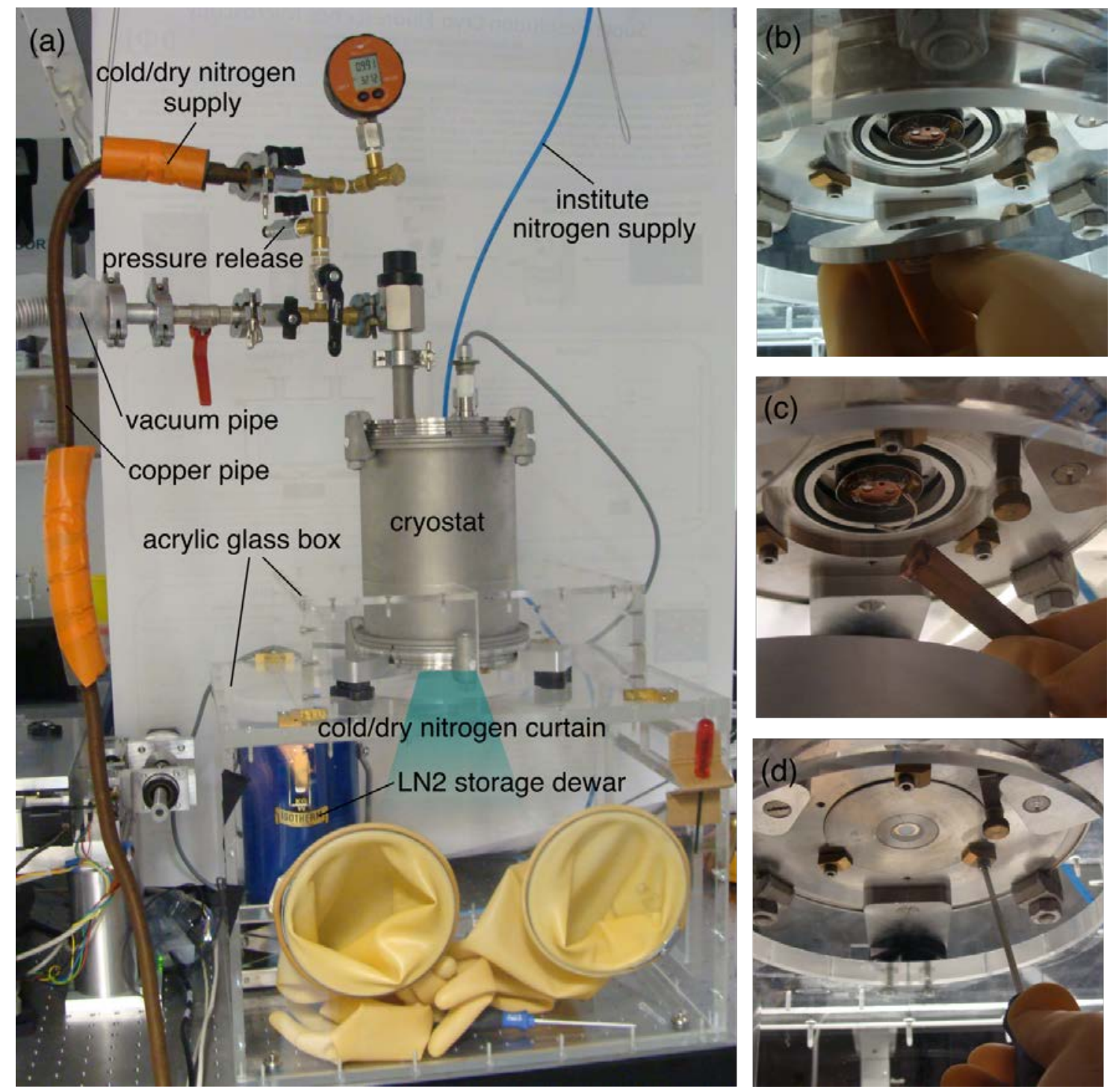

Figure 23: Sample transfer at CT. (a) A cold cryostat sitting in the workstation for sample transfer. (b) Opening the window disc. (c) Sample transfer under cold nitrogen gas curtain. (d) Closing the window disc.

The workstation consists of two acrylic glass boxes (Fig. 23 a), which insulate the cryostat from the humid lab environment. These boxes are constantly flushed with nitrogen gas from the institute's supply, which creates a relative dry environment with a humidity of $\sim 20 \%$ (v.s. lab humidity of $\sim 85 \%$ in June in Göttingen). A cold cryostat - either pre-cooled for sample loading, or containing a sample for unloading - is kept in place in the workstation via 
kinematic mounts. A vacuum pipe and a nitrogen supply pipe are connected to the cryostat for operation.

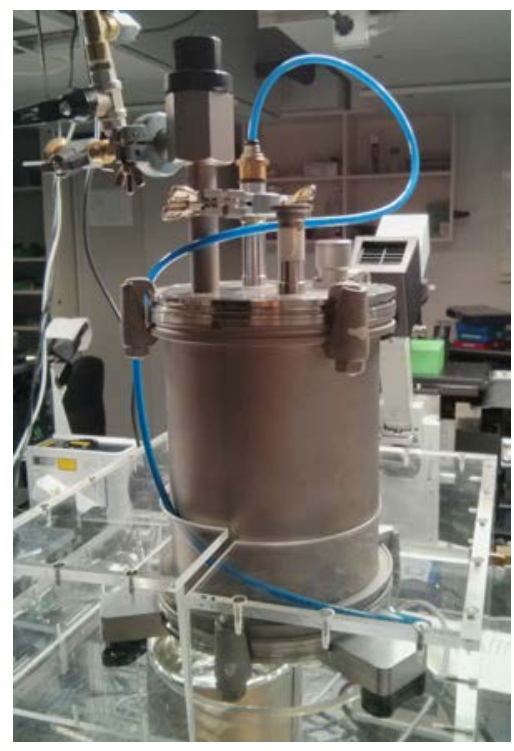

(a)

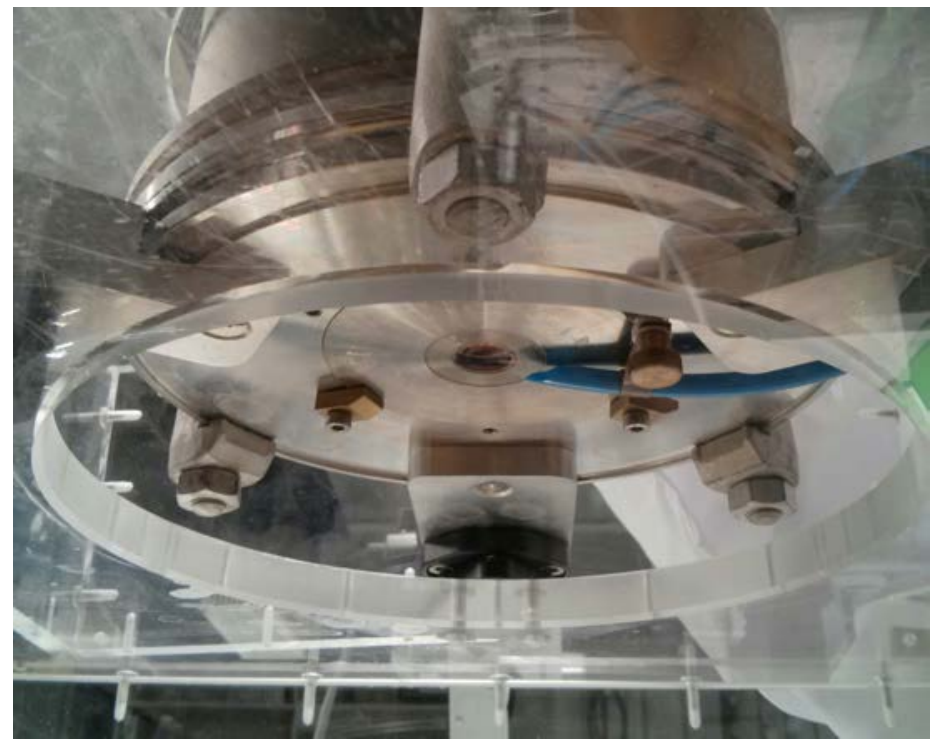

(b)

Figure 24: Nitrogen purging on the optical window. (a) Cryostat with a purging pipe (blue) connected to the outlet channel of the LN2 tank. (b) Purging the optical window with dry nitrogen gas keeps it clear from ice condensation.

Prior to sample transfer, the LN2 from the dewar is streamed through a long copper pipe, which heats up the LN2 to its gaseous form by the ambient temperature. The nitrogen gas extracted directly from its liquid form can be considered as "100\% dry". This dry nitrogen gas is gently supplied to the vacuum chamber. When the pressure inside the cryostat reaches atmospheric pressure, the window disc can be opened (Fig. 23 b). Next, a stronger LN2 flow is immediately supplied, and the cold nitrogen gas flushes down around the sample, forming a "gas curtain". Under this curtain, the sample can be safely transferred to/from a small LN2 storage dewar (Fig.23c). After the sample transfer is done, the nitrogen curtain is retracted by terminating the LN2 supply. Finally, the window disc is quickly closed (Fig.23d), and the vacuum is regenerated. During the whole procedure, the temperature of the sample mount is kept below $115 \mathrm{~K}$ (measured by imbedded thermal sensor).

This sample transfer procedure prevents ice condensation on the sample and on the inner parts of the cryostat. However, flushing the cryostat with cold nitrogen gas also cools down the vacuum housing to below $0^{\circ} \mathrm{C}$. If directly removing the cryostat from the dry workstation and placing it back to the microscope, ice will form on the outer surface of the vacuum housing, as well as on the optical window. There are two possible ways to avoid 
this. (i) One can heat up the vacuum housing to above $0{ }^{\circ} \mathrm{C}$ in the workstation using e.g. a hair drier, and then place the cryostat back to the microscope. (ii) A flexible pipe can be connected to the outlet channel of the LN2 tank (Fig.24a), and guides the evaporated dry nitrogen gas to purge gently on the optical window (Fig.24b). This also keeps the window locally clear.

When comparing this procedure to sample transfer at RT, CT transfer demands more efforts and experiences, and takes a longer time of about $\sim 30 \mathrm{~min}$ to finish. Flushing the nitrogen curtain also consumes more LN2. Therefore, it is in general preferred to prepare the sample in dry form, or in glycerol/PVA, so that the convenient RT transfer can be applied.

\subsection{Gas supply}

The sample is located in a high vacuum of $\sim 10^{-3} \mathrm{~Pa}$ in the cryostat. The lack of oxygen influences certain chemical properties of the fluorophore. For example, Atto488 and Alexa488 dyes are trapped in dark states in vacuum due to the absence of oxygen (section 7.3).

To provide the possibility of feeding certain gases to the sample, a piping system has been designed as an add-on to the cryostat (Fig.25). This piping system contains a vacuum pump port, a gas supply inlet, and a pressure release outlet. Each channel is equipped with a valve for separate control. This system is connected to the vacuum valve of the cryostat, and feeds gasses into the vacuum chamber. The gas pressure is measured by the internal barometer in the turbo pump, and is controlled manually by adjusting the valves. The manual control can be automated using commercially available pressure controllers, which provide more convenience and higher precision. This piping system is also used for sample transfer at CT, as described in section 5.2 .

The supplied gas has to be "100\% dry" to avoid ice contamination of the sample. Oxygen and nitrogen extracted directly from their liquid forms have been tested to meet this condition. One challenge of supplying gases to the vacuum space is that it reduces the thermal insulation, and thus disturbs the thermal stability of the system. This brings about several side-effects. (i) The temperature on the sample may rise due to the heat transfer through convection. (ii) LN2 evaporation will be accelerated, thus the total duration of CT will decrease. (iii) The optical window will be cooled down to below $0^{\circ} \mathrm{C}$, and ice will form on the outer surface of the window. (This effect can be eliminated by gas purging on the window, as shown in Fig. 24. 


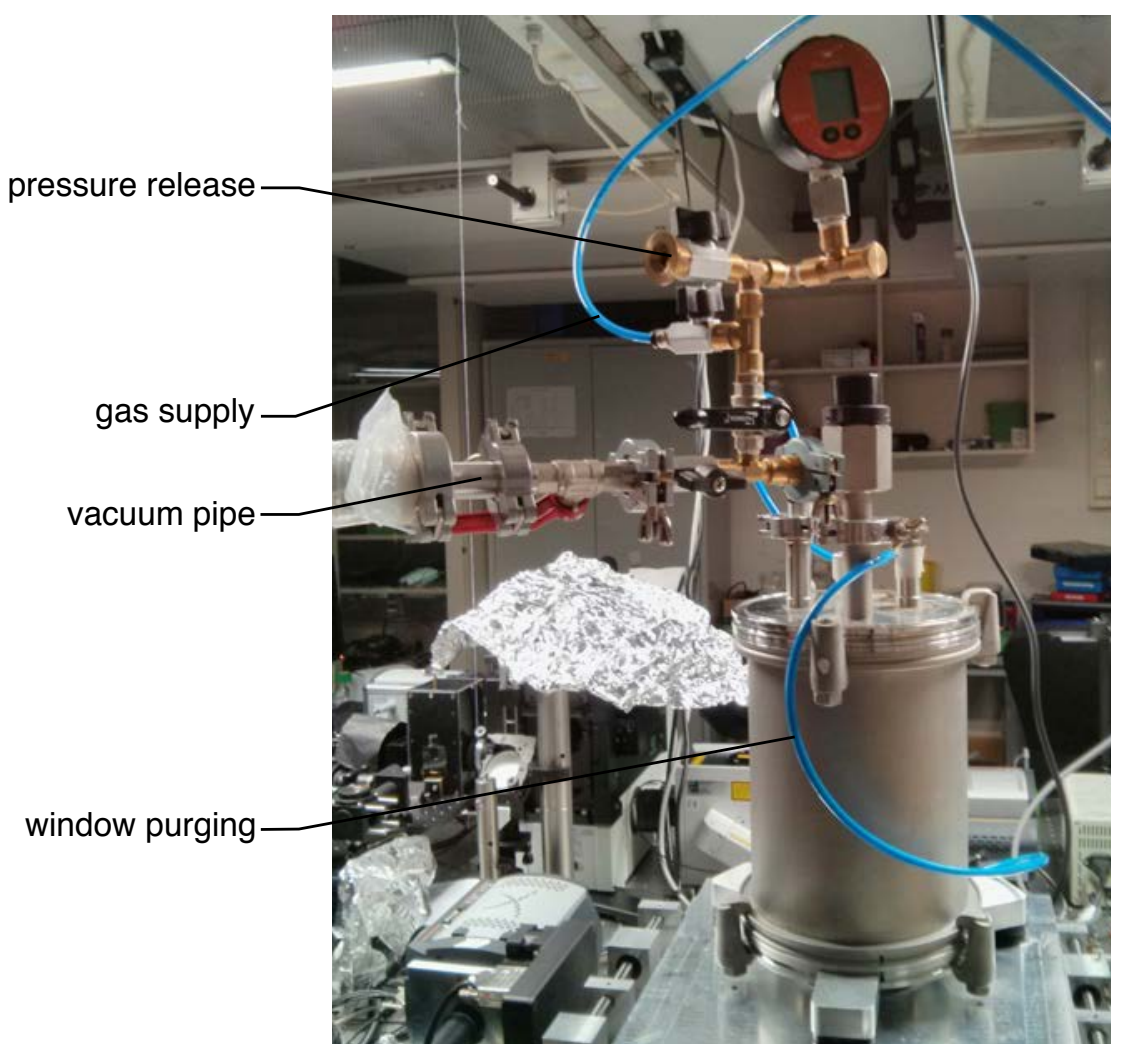

Figure 25: Piping system for gas supply.

However, in most of the cases, only a very little amount of gases is needed, e.g. to quench the dark state of Atto488 and Alexa488 dyes. This little amount of gas has rather minor influence on the thermal stability, but brings important chemical effects to the sample. Therefore, supplying gasses to a vacuum-insulated cryostat is worth applying, having only small impact on the thermal stability. 


\section{Part II}

\section{ORGANIC DYE PROPERTIES AT $89 \mathrm{~K}$}

This part of the thesis explores the properties of commonly used organic dyes at $89 \mathrm{~K}$. Section 6 demonstrates the photostability enhancement upon cooling to CT. Section 7 investigates the influence of low temperature and vacuum on the blinking behaviour. Section 8 examines the fluorescence spectrum at $89 \mathrm{~K}$. 


\section{Photostability}

\subsection{Photostability enhancement at $89 \mathrm{~K}$}

Photobleaching is one of the major challenges for fluorescence microscopy. It limits the number of photons that can be detected from a sample. The limited photon yield is the bottleneck to achieve a higher resolution for all fluorescence microscopy techniques. It is known that the photostability of organic dyes will be increased upon cooling to CT, which results in a much higher photon yield. However, most of the existing studies are based on ensemble measurements. In this thesis, the photostability of organic dyes was examined on the single molecule level at $89 \mathrm{~K}$.

Alexa647, Atto674N, Atto655, and Cy5 dyes have been tested for photostability at $89 \mathrm{~K}$. These red dyes were chosen for multiple reasons. (i) They have high brightness, thus are suitable for single molecule detection using a low NA air objective. (ii) They are very well developed and commercialized, and have been widely used in research, especially in the field of super-resolution microscopy. (iii) The red channel (e.g. with $637 \mathrm{~nm}$ excitation) is almost free from fluorescent impurities, which is crucial for single molecule experiments. The blue channel (e.g. with $488 \mathrm{~nm}$ excitation) and the green channel (e.g. with $532 \mathrm{~nm}$ excitation) often show impurity signals, which can hardly be totally removed. (iv) Alexa488 and Atto488 are excluded from this test, because they will be trapped in the dark state in vacuum (section 7), thus it is difficult to examine them on a single-molecule level.

Among the chosen dyes, Atto647N was taken as a representative for a detailed report in this section. To investigate the photostability enhancement, a photobleaching test was carried out at RT $(294 \mathrm{~K})$ and CT $(89 \mathrm{~K})$ on the single molecule level. An Atto647N dye solution ( $100 \mathrm{pM}$ ) was spin-coated on a cleaned silica coverslip, forming a layer of single molecules. The sample was excited using a $637 \mathrm{~nm} \mathrm{CW}$ laser with an irradiance of $\sim 300 \mathrm{~W} / \mathrm{cm}^{2}$. Consecutive series of images were recorded at $294 \mathrm{~K}$ (movie 1, extracted frames shown in Fig.26.a) and at $89 \mathrm{~K}$ (movie 2, extracted frames shown in Fig. 26 b) with a frame rate of $0.5 \mathrm{~Hz}$ and $1 \mathrm{~Hz}$, respectively.

To quantify the photobleaching, the number of molecules in each frame was counted. As shown in Fig. 26 (c), at $294 \mathrm{~K}$, almost all molecules were bleached after about three minutes, whereas at $89 \mathrm{~K}$, still $\sim 31 \%$ molecules remained fluorescent after about four hours. The ratio between the half-life period at $294 \mathrm{~K}(\sim 20 \mathrm{~s})$ and at $89 \mathrm{~K}(\sim 7600 \mathrm{~s})$ suggested a $\sim 380$-fold photostability enhancement. 
(a)
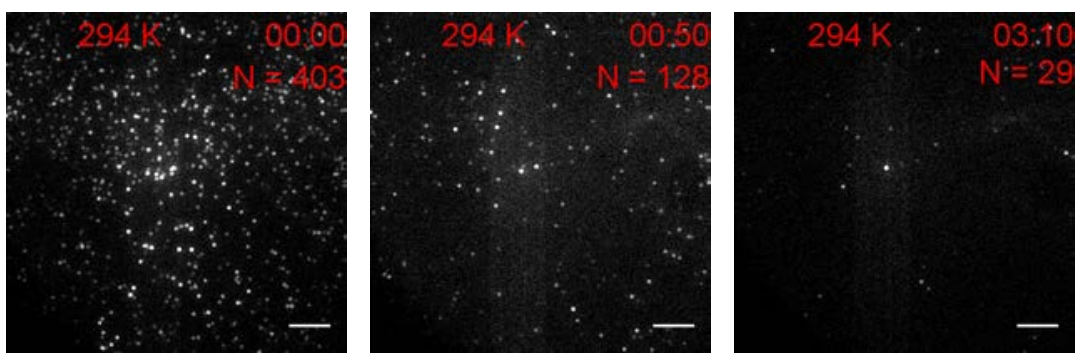

(b)
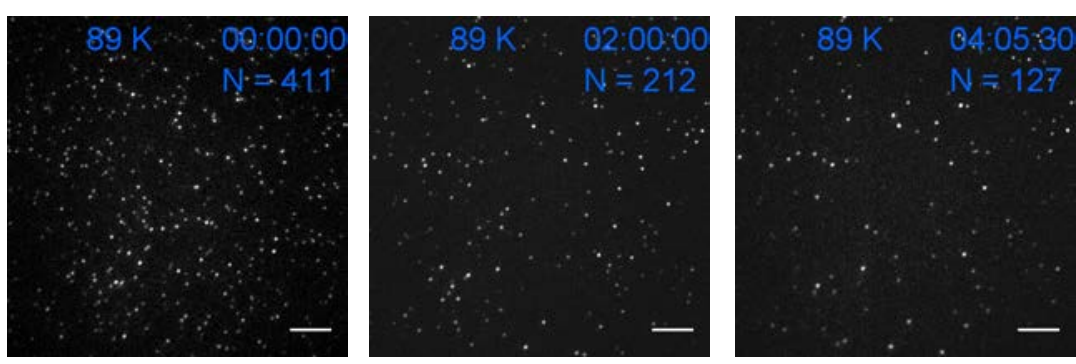

(c)

(d)
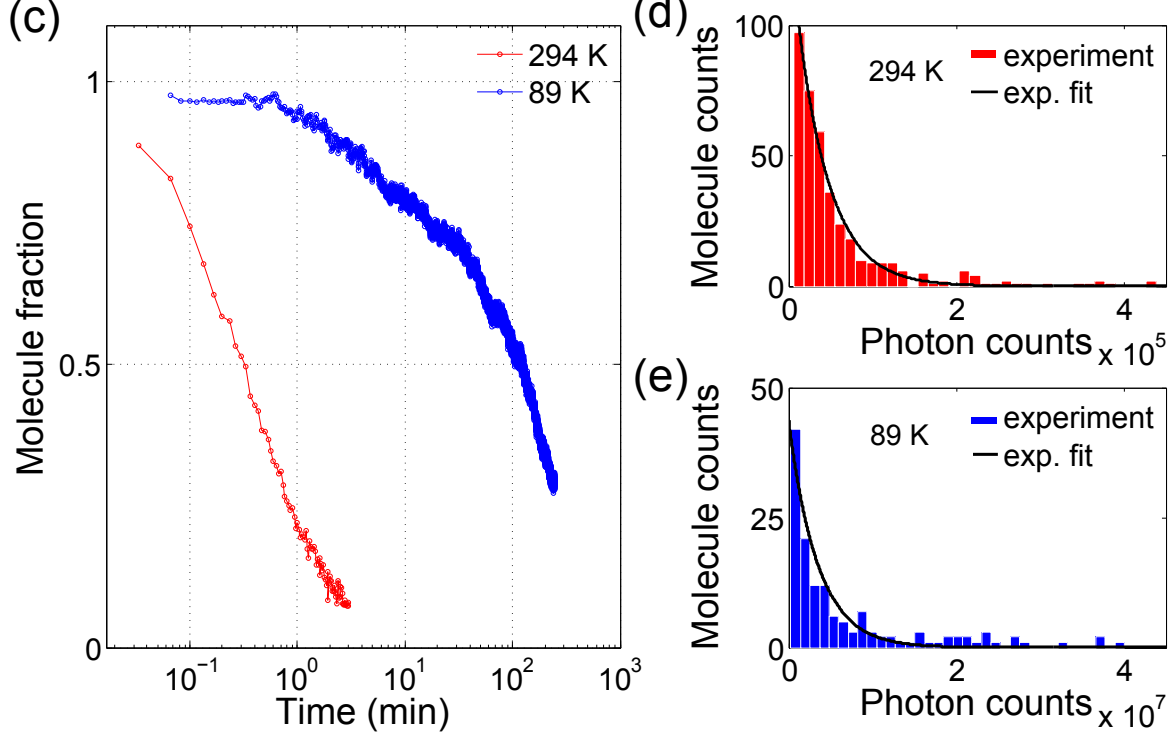

Figure 26: Photobleaching of Atto647N molecules at $294 \mathrm{~K}$ and $89 \mathrm{~K}$. (a, b) The first, middle, and last frame of movie 1 and movie 2. Scale bar: $10 \mu \mathrm{m}$. (c) Comparison of photobleaching between $294 \mathrm{~K}$ and $89 \mathrm{~K}$. Number of molecules in each frame of the movie was counted, and the fraction with respect to the first frame was calculated. (d, e) Total number of detected photons per molecule till photobleaching. The molecules in the last frame of movie 2 were excluded from this analysis. The histograms of the photon count were fitted to an exponential decay. Data analysis assisted by Simon Stein.

Alternatively, the number of photons detected from each single molecule till photobleaching was determined. To obtain the photon numbers, the PSF of each molecule was fitted to a 2D Gaussian function using the rapidSTORM [70] software. The fitted amplitude $A$ was converted to photon counts $N$ using a formula provided by the camera manufacturer in the form of $N=(A-b) s / g$, where $b$ is the camera bias, $s$ the device's specific sensitivity, and 
$g$ the EM-gain. The photon count $N$ was summed up over all frames for each molecule to obtain the total photon yield.

As shown in Fig.26(d) and (e), at both temperatures, the histogram of the photon yield exhibited an exponential decay, as expected from the theoretical prediction. The average photon yield increased from $3.8 \times 10^{4}$ at $294 \mathrm{~K}$ to $3.5 \times 10^{6}$ at $89 \mathrm{~K}$, suggesting an increase by two orders of magnitude.

A similar photostability enhancement was observed for Atto655, Alexa647, and Cy5 dyes as well. These results clearly indicate that the photostability of organic dyes can be drastically enhanced upon cooling to LN2 temperature of $89 \mathrm{~K}$, delivering an average photon yield of several millions per molecule. This large amount of photons opens fascinating possibilities for many new applications, e.g. the localization of single molecules with exceptional high precision, as described in section 9 .

\subsection{Photostability at different excitation powers}

Besides temperature, another factor which influences the photobleaching is the excitation power. At RT, higher excitation power leads to faster photobleaching and less photon yield. This effect is examined at $89 \mathrm{~K}$ in this section.

Photobleaching experiments described in the previous section (Fig.26) were repeated at four excitation powers at $89 \mathrm{~K}$. As shown in Fig. 27, when increasing the excitation power, photobleaching became faster (27]a), and the histogram of the photon yield shifted towards zero (Fig. $27 \mathrm{~b}$ ). The resulting average total photon yield decreased with exponential decay (Fig. 27]c).

These results suggest that at $89 \mathrm{~K}$, increasing the excitation power does not only accelerate the photobleaching process, but also reduces the total photon yield. Therefore, a high excitation power is not preferable if a large photon number is desired, e.g. for high precision localization. Moreover, a higher excitation power also results in a faster and larger sample drift, which is especially troublesome for experiments with long exposure time, as the sample will shift during the exposure. 
(a)

(c)
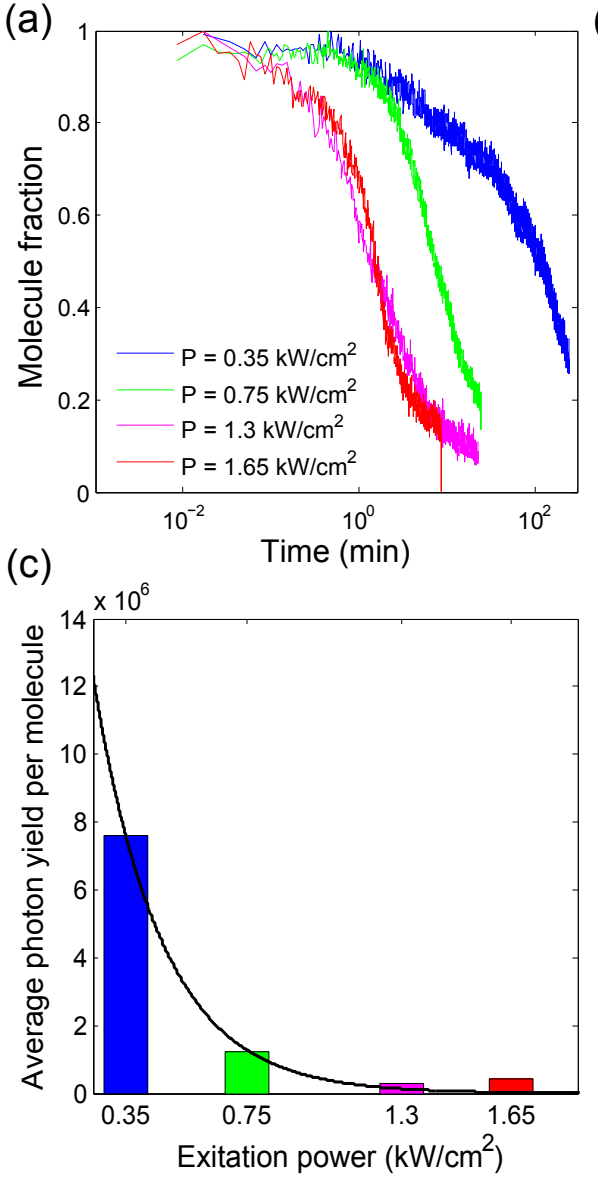

(b)
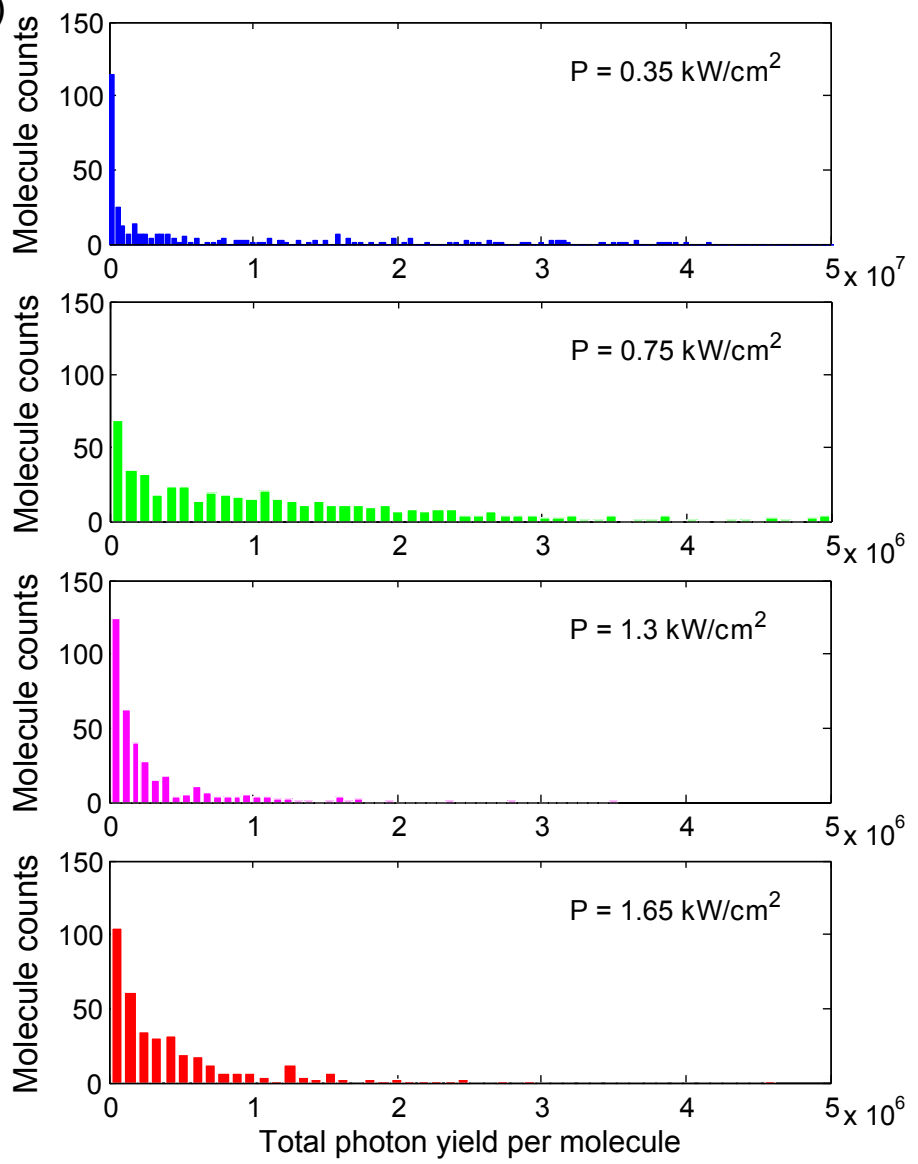

Figure 27: Photon yield at different excitation powers at $89 \mathrm{~K}$. (a) Photobleaching of Atto647N molecules at four excitation powers. Experiments and analysis were performed as described in Fig. 26. (b) Histogram of the total photon yield per molecule, determined as described in section 6.1. Notice the different scale of the $\mathrm{x}$ axis in the first plot. (c) Average photon yield at four excitation powers, fitted to an exponential decay. Data analysis assisted by Simon Stein.

\section{$7 \quad$ Blinking}

\subsection{Introduction}

Blinking is an intrinsic property of many fluorescent molecules, such as organic dyes, fluorescent proteins, quantum dots, silver/gold nano-particles, etc. The underlying mechanism is typically associated with the dark state (e.g. triplet) dynamics of the fluorophore. Blinking has given rise to many important applications, especially in the field of super-resolution SMLM, such as (d)STORM or PALM. These techniques reduce the blinking rate (on/off ratio) of organic dyes or fluorescent proteins in such a way that only a sparse subset of molecules 
are fluorescent during each image acquisition. The sparsity assures no PSF overlapping of the "on" molecules in each frame, and single molecules can be localized individually with a precision far below the diffraction limit.

Various approaches have been developed to control the blinking behaviour of fluorescent molecules. For example, for fluorescent proteins, tremendous efforts have been invested in adjusting the fine structures such that the proteins can be switched on/off by light of different wavelengths 12171 . For organic dyes, the blinking can be either photon-controlled by placing a "switching assistant molecule" close to the target dye (STORM 4 ] $]$, or can be chemically induced by adding certain substances (e.g. aliphatic thiols) to the imaging buffer $\left(\mathrm{dSTORM}^{[2]}\right)$. For both approaches, an oxygen scavenging buffer system is necessary to prolong the life-time of the dark state.

Interestingly, it was found in this project that the particular environment of vacuum and low temperature also has strong influences on the blinking behaviour of organic dyes. The tested dyes can be categorized into two groups regarding two different blinking behaviors under these conditions. (i) The blinking of Alexa647, Atto647N, and Cy5 molecules is slowed down by $\sim 10$ fold, which is described in section 7.2, (ii) Alexa488 and Atto488 molecules are trapped in the dark state, and return to the fluorescent state in a stochastic manner, which is described in section 7.3 .

\subsection{Blinking of Alexa647, Atto647N, and Cy5 dyes}

Alexa647, Atto647N, and Cy5 are the most commonly used dyes for SMLM. Among these dyes, Alexa647 was chosen for a thorough examination of the blinking behaviour at CT.

To examine the influence of vacuum and low temperature on the blinking behaviour separately, movies of single Alexa647 molecules were taken with $50 \mathrm{~ms}$ exposure time in the cryostat in three conditions (movie 3): (i) air, $293 \mathrm{~K}$; (ii) vacuum, $293 \mathrm{~K}$; (iii) vacuum, $89 \mathrm{~K}$. The intensity traces of each molecule were extracted from the movies, and the duration of the "on" and "off" time (in the unite of frame) was histogrammed for all the traces in each movie.

As shown in Fig. 28, the average "on" time of Alexa647 molecules was shortened by about three times $(9.9 / 3.1)$ in a vacuum of $\sim 10^{-2} \mathrm{~Pa}$, and was further reduced by about 1.4 times $(3.1 / 2.2)$ when cooling down to $89 \mathrm{~K}$. On the other hand, the "off" time was extended by a factor of 1.4 and 1.3 correspondingly. As a result, the final on/off ratio was reduced by ca. 
ten times from 3.3 to 0.37 in vacuum at $89 \mathrm{~K}$.

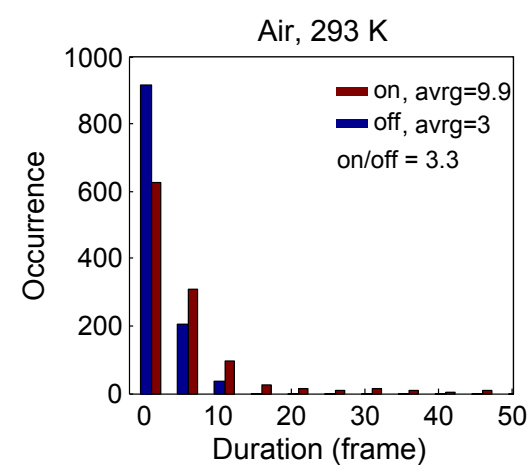

(a)

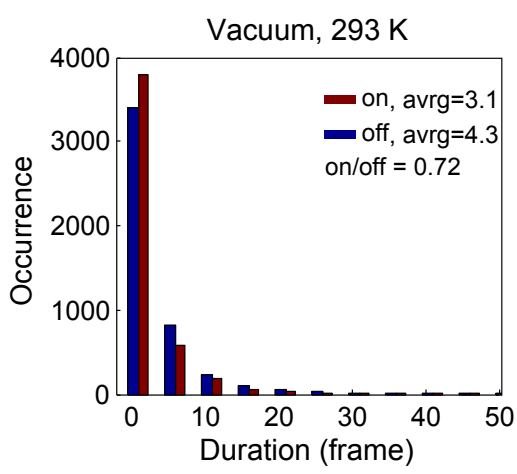

(b)

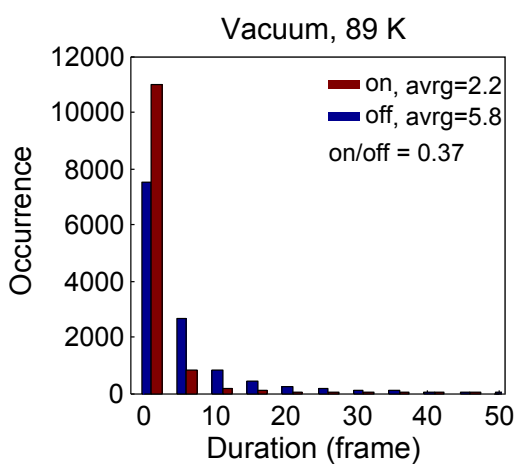

(c)

Figure 28: Blinking of Alexa647 molecules. Single Alexa647 molecules were imaged with $50 \mathrm{~ms}$ exposure time and 30 EM-gain in three conditions: (a) air, $293 \mathrm{~K}$; (b) vacuum, $293 \mathrm{~K}$; (c) vacuum, $89 \mathrm{~K}$. The duration of the on and off frames was determined from the single molecule intensity traces, and was histogrammed for all the molecules. The arithmetic mean of the on and off time was used to compute the on/off ratio. Data analysis assisted by Simon Stein.

The reduction of the on time and the corresponding extension of the off time in vacuum can be attributed to the removal of oxygen. It has been well known that oxygen is a strong triplet state quencher. Therefore, in vacuum, the absence of oxygen strongly prolongs the life-time of the dark state. Furthermore, when cooling down to $89 \mathrm{~K}$, the vibrational energy of the molecules will be reduced, which decreases the transition probability from the dark state to the ground state, thus further extending the dark state duration.

The final on/off ratio of 0.37 (less than 0.5 ) indicates that the probability for a molecule to be in the on state is less than one half. This means that if there are only two molecules located within diffraction-limited spot, they can be separated in the temporal domain. This provides a new possibility to colocalize two molecules with very high precision (resolution), which is discussed in section 10.4 .

The slowing down of the blinking has also been observed for Atto647N (movie 4, $50 \mathrm{~ms}$ exposure) and Cy5 molecules (movie 5, $10 \mathrm{~ms}$ exposure). Remarkably, Cy5 has a higher brightness than Alexa647 and Atto647N, thus allowing for a very short exposure time of $10 \mathrm{~ms}$ without scarifying the $\mathrm{s} / \mathrm{n}$ ratio. 


\subsection{Blinking of Atto488 and Alexa488 dyes}

Unlike Alexa647, Atto647N, and Cy5 dyes, Atto488 and Alexa488 dyes were trapped "firmly" in the dark state in vacuum due to the lack of oxygen. As demonstrated in Fig.29, when placing Atto488 molecules in a vacuum of $\sim 3.4 \times 10^{-2} \mathrm{~Pa}$ in the cryostat (at RT), almost all the molecules became non-fluorescent. After feeding pure oxygen into the cryostat, the molecules returned to the fluorescent state. This result indicates that these fluorophores possess a long-living dark state, the duration of which can be drastically extended by removing oxygen.

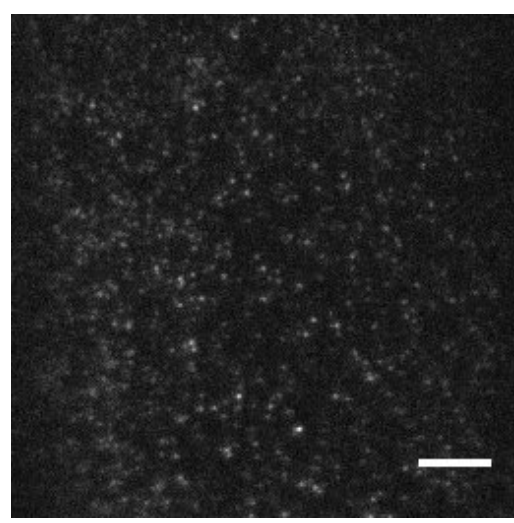

(a) air

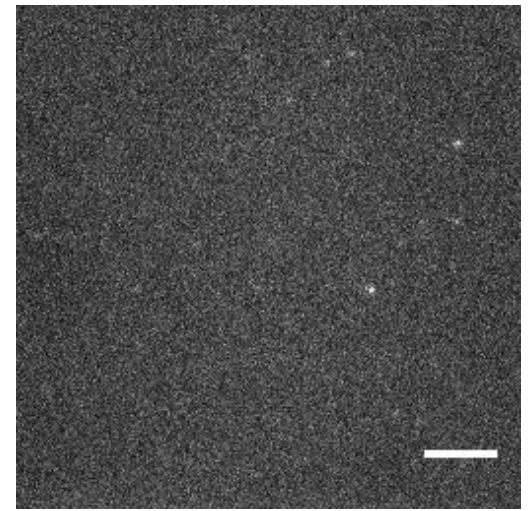

(b) vacuum

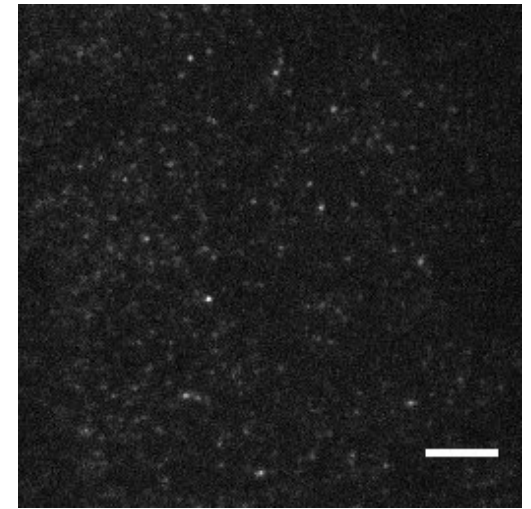

(c) oxygen

Figure 29: Atto488 molecules in vacuum. Atto488 molecules were imaged on a silica coverslip in the cryostat in three conditions: (a) in air; (b) in a vacuum of $3.4 \times 10^{-2} \mathrm{~Pa}$ (measured at integrated barometer in the turbo pump); (c) in pure oxygen. Images were taken with $473 \mathrm{~nm}$ excitation, $0.1 \mathrm{~s}$ exposure time, and 100 EM-gain. Scalebar: $10 \mu \mathrm{m}$.

This result suggests the possibility of performing STORM at CT using Atto488 and Alexa488 dyes. To demonstrate cryo-STORM, Atto488 dye solution with a relative high concentration was dried on a coverslip, and the sample was imaged in vacuum at $89 \mathrm{~K}$ at $473 \mathrm{~nm}$ laser excitation with an irradiance of $\sim 0.75 \mathrm{~kW} / \mathrm{cm}^{2}$. A movie was taken with $50 \mathrm{~ms}$ exposure time and 100 EM-gain. As shown in movie 6 (extracted frames displayed in Fig. 30), upon laser irradiation, the molecules were quickly pushed into their dark states, and only a sparse subset stochastically returned to the fluorescent states. This process lasted for over 12000 frames (only the first 500 frames shown in the movie) until all the molecules in the "dark state reservoir" switched on and photobleached. Similar behaviour has been also observed for Alexa488 molecules, as shown in movie 7.

These results suggest that STORM can be directly applied in the absence of oxygen at $89 \mathrm{~K}$, without the need of any imaging buffer. The vacuum plays the role of the oxygen- 
scavenging buffer for conventional (d)STORM, and the low temperature delivers enhanced photostability of the dye molecules. Under these conditions, stochastic photoswitching with sufficient sparsity can be automatically achieved using Atto488 and Alexa488 dyes. These dyes are pushed into the dark state and are sparsely read out at the same laser wavelength.

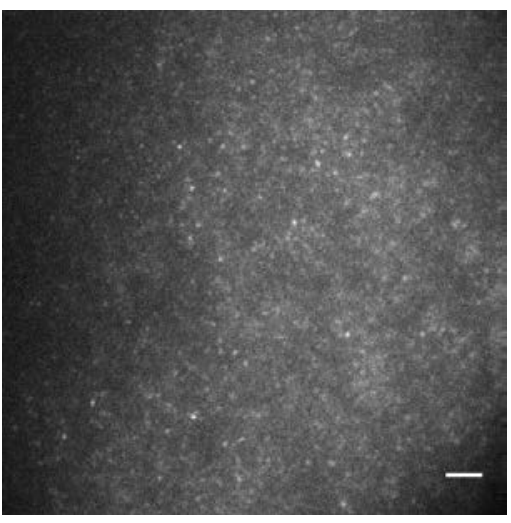

(a) Frame 1

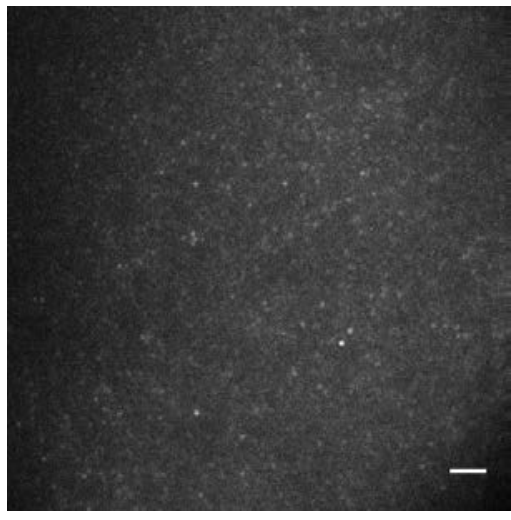

(b) Frame 10

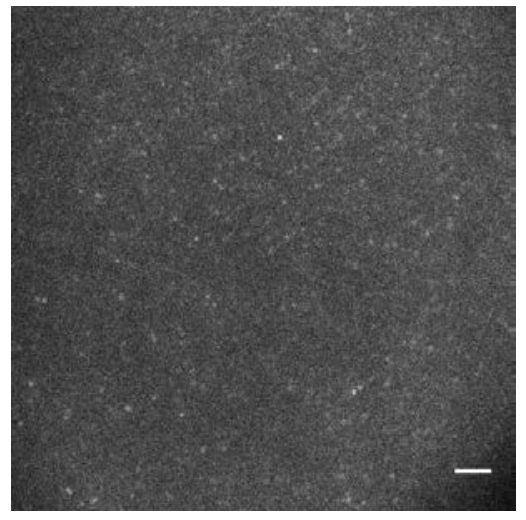

(c) Frame 100

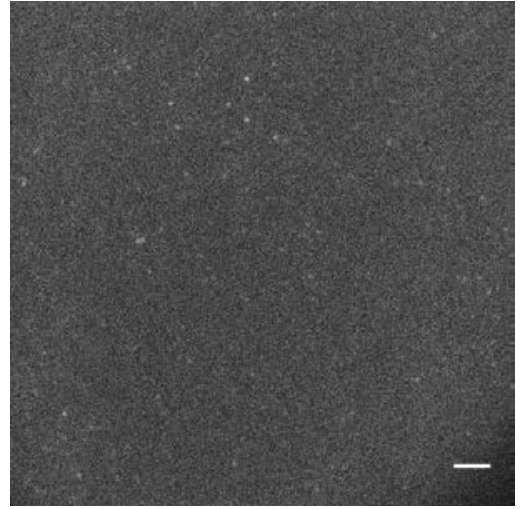

(d) Frame 1000

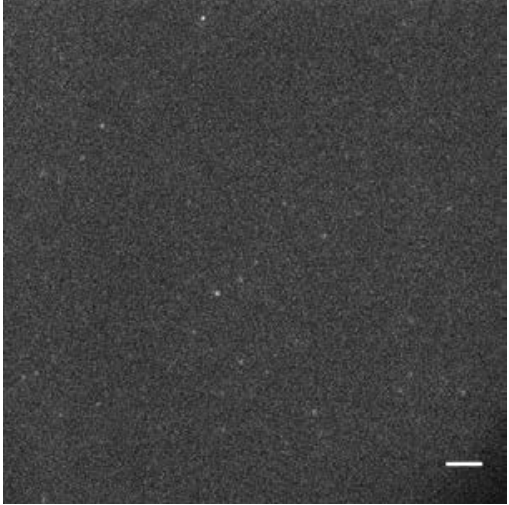

(e) Frame 10000

Figure 30: Stochastic photo-switching of Atto488 molecules at $89 \mathrm{~K}$. Five frames were extracted from the full-length data of movie 6 (first 500 frames shown in the movie), demonstrating the off-switching of the Atto488 molecules upon laser irradiation at $473 \mathrm{~nm}$, and the sparse stochastic on-switching using the same laser. Images were taken with $50 \mathrm{~ms}$ exposure time and 100 EM-gain. Scalebar: $10 \mu \mathrm{m}$.

As for STORM at RT, a key factor that assures the stochastic switching is sufficiently large excitation power. When reducing the irradiation intensity used in movie 6 from $\sim$ $0.75 \mathrm{~kW} / \mathrm{cm}^{2}$ to $\sim 0.2 \mathrm{~kW} / \mathrm{cm}^{2}$, the stochastic switching was suppressed. As shown in movie 8, many molecules remained constantly on, and only a few molecules switched from the dark state to the fluorescent state.

The prolonged triplet state, however, is not favorable for some other microscopy techniques. 
For example, cryo-STED has been attempted at $76 \mathrm{~K}$ in a vacuum cryostat 47 . In this approach, the build-up of the triplet state population becomes the major challenge for STED at CT, because it reduces drastically the brightness of the sample. The authors attributed the prolongation of the triplet state to the low temperature of $76 \mathrm{~K}$, and introduced a method to depopulate the triplet state by illuminating the fluorophore with an additional laser that matches the transition energy between the triplet state and the ground state, similar to the "stimulated emission depletion" from the excited state to the ground state using a STED laser. However, the results in this section indicate that the absence of oxygen is more likely the dominant reason for inhibiting the triplet state depopulation. Thus, another and easier way to quench the triplet state is to feed a little amount of pure oxygen to the sample in vacuum, which can be done using the cryostat developed in this project (section 5.3).

\section{Fluorescence Spectrum}

The spectrum of fluorescent molecules narrows down to a single line when cooling to the LHe temperature of $4 \mathrm{~K}^{5}$. The distinct separation of single-molecule spectra at $4 \mathrm{~K}$ can be also employed to distinguish between different types of molecules in cryo-FM $\underline{63}$. However, fluorescence spectra at LN2 temperatures have not be thoroughly examined. In this thesis, the emission spectra of several commonly used organic dyes were measured at $89 \mathrm{~K}$ in ensemble. To include the possible influence of the absence of oxygen, the spectra were also measured in vacuum at RT as a reference.

To measure the emission spectrum, solutions of organic dyes (Alexa488, Atto488, Alexa647, Atto647N, and Atto655) were deposited onto silica coverslips, and the samples were loaded into the cryostat. The emission spectrum was measured in three conditions: (i) air, $293 \mathrm{~K}$; (ii) vacuum, $293 \mathrm{~K}$; (iii) vacuum, $89 \mathrm{~K}$. As shown in Fig.8, except for the Atto488 dye, all other tested dyes exhibited no evident change in the spectrum upon the removal of oxygen or cooling down to $89 \mathrm{~K}$. This result suggests that the temperature of $89 \mathrm{~K}$ is not low enough to "freeze out" the vibrational states of the fluorophores, thus does not affect the emission spectrum. The spectrum of Atto488 dye displayed a minor change around $670 \mathrm{~nm}$, far away from the emission maximum. This is possibly due to the lack of oxygen which hinders the depopulation of the triplet state, as discussed in section 7.3 .

The unchanged emission spectrum at $89 \mathrm{~K}$ indicates a minimal change of the chemical properties of the fluorophore. This is beneficial for cryo-FM, because the high brightness and the enhanced photostability of the dyes is sustained at $89 \mathrm{~K}$. Moreover, many super-resolution 
techniques which strongly rely on the chemical properties of fluorophores will still work at 89 K. For example, cryo-PALM (using FPs) has been demonstrated recently to super-resolve mitochondria membranes by Xu and colleagues at LN2 temperatures ${ }^{54}$. Cryo-STORM (using organic dyes) was shown to be applicable at $89 \mathrm{~K}$ in this project (section 7.3).
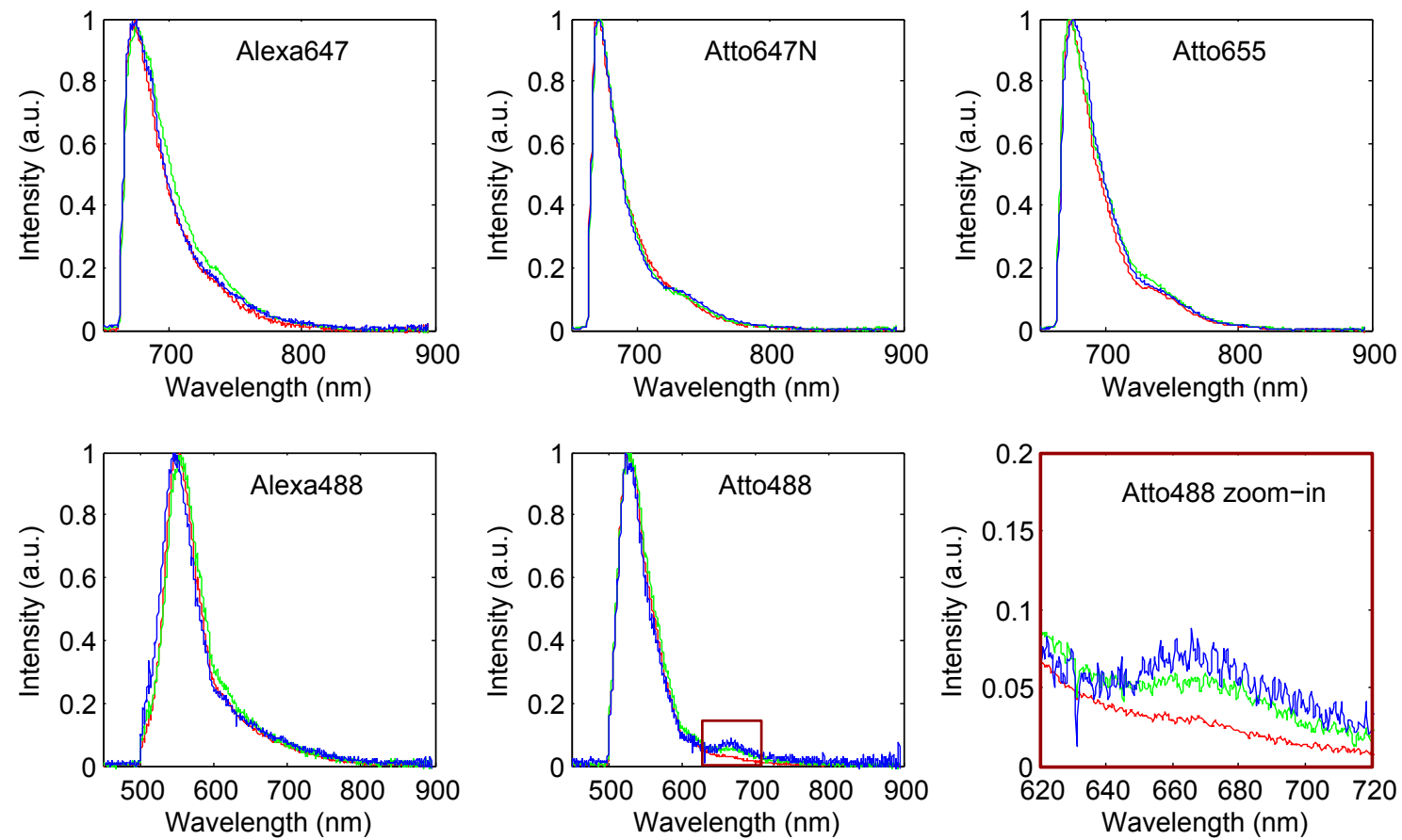

air, $293 \mathrm{~K}$

vacuum, $293 \mathrm{~K}$

vacuum, $89 \mathrm{~K}$

Figure 31: Emission spectrum of organic dyes in vacuum at $89 \mathrm{~K}$. Emission spectra of several organic dyes were measured using a spectrometer (2048-USB2-FCPC, Avantes) with $1 \mathrm{~s}$ integration time in three conditions: (i) air, $293 \mathrm{~K}$; (ii) vacuum $\left(\sim 10^{-2} \mathrm{~Pa}\right), 293 \mathrm{~K}$; (iii) vacuum $\left(\sim 10^{-3} \mathrm{~Pa}\right)$, 89 K. Atto647N, Atto655, and Alexa647 dyes were measured under $637 \mathrm{~nm}$ laser excitation through a $665 \mathrm{~nm}$ long pass filter (665 LP ET, Chroma). Atto488 and Alexa488 dyes were measured under $473 \mathrm{~nm}$ laser excitation through a $500 \mathrm{~nm}$ long pass filter (500 LP ET, Chroma). 


\section{Part III}

\section{APPLICATIONS}

This part of the thesis presents two applications of the cryo-fluorescence microscopy system. Section 9 demonstrates single molecule localization with subnanometer precision. Section 10 discusses several possibilities for dual molecule colocalization with single nanometer resolution. 


\section{Single Molecule Localization}

The superior mechanical stability of the cryo-imaging system and the drastically enhanced photon yield of organic dyes at $89 \mathrm{~K}$ allow for the localization of single molecules with an exceptional sub-nanometer precision. A robust procedure was developed in this project to correct for sample drift, which determines the localization precision,.

To demonstrate this procedure, the full-length data of movie 2 was used as an example. First, the movie was analyzed using the freeware rapidSTORM ${ }^{[0]}$, which returned the localizations of single molecules in each frame. An overlay of these localizations reflected the lateral sample drift (Fig.32 a). To correct for this drift, single molecule tracking was performed, and the frame-to-frame displacement was averaged for all molecules, delivering the average sample drift (Fig. 32 b). This drift was subtracted from the original positions of the molecules frame by frame. The resulting drift-corrected positions constituted well-defined clusters (Fig. $32 \mathrm{c}$ ). The histogram of these clusters (Fig. 32 f) could be well fitted with a nearly circular Gaussian distribution (Fig. $32 \mathrm{~g}$ ), with an ellipticity between zero and $10 \%$. The center position of each Gaussian fitting determined the position of the single molecule. The localization precision was given by the standard error of mean $\sigma_{S E M}$ of the cluster averaged over $2 \mathrm{D}$ using equation:

$$
\sigma_{S E M}=\frac{\sigma_{1}+\sigma_{2}}{2 \sqrt{N}}
$$

where $\sigma_{1}$ and $\sigma_{2}$ are the standard deviations along the two principal axes of the cluster, and $N$ is the number of localizations.

As the number of localizations $N$ differed from molecule to molecule, the determined localization precisions $\sigma_{S E M}$ also displayed a distribution, as shown in Fig.33(a). The relationship between the localization precision and the number of detected photons agreed excellently with the theoretical predictions derived by Mortensen et al. $\frac{73}{7}$ The statistics showed that more than $80 \%$ of the molecules were localized with a precision below one nanometer. The same analysis was applied to the full-length data of movie 1 measured at RT (Fig. $33 \mathrm{~b}$ ). The comparison showed a clear ten-fold improvement in the localization precision at $89 \mathrm{~K}$.

The success of achieving the exceptional sub-nanometer localization precision relies on the superior mechanical stability of the imaging system, which delivers moderate post-correctable lateral drift and negligible axial drift over many hours. To correct for the lateral drift in this experiment, the single molecules themselves were used as markers. For other applications, fiducial markers (e.g. fluorescent beads) can be added to the sample to track the drift. As 
no active online drift compensation (e.g. focal lock) is needed, the setup and the operation are greatly simplified.

Remarkably, the determined sub-nanometer localization precision is already on the scale of the size of a fluorophore, and is below the typical length of linkers used for dye labeling of biological samples. This means that at $89 \mathrm{~K}$, the photon yield is no longer the bottleneck that limits the final localization precision. The new bottleneck is rather the size of the fluorescent marker and the labeling technique. Therefore, there is no advantage to further increase the localization precision, e.g by cooling the sample to a lower temperature using LHe.
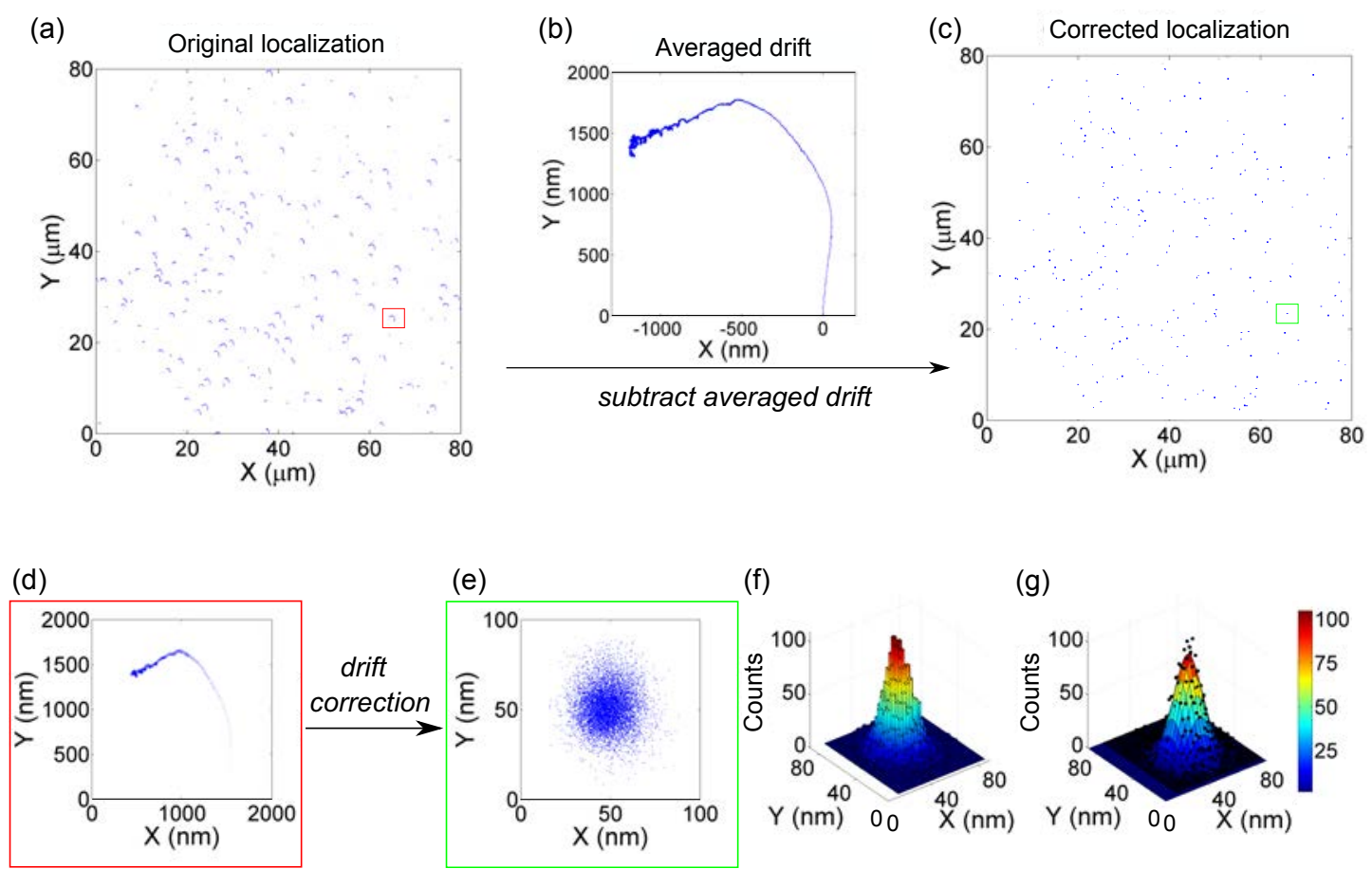

(e)

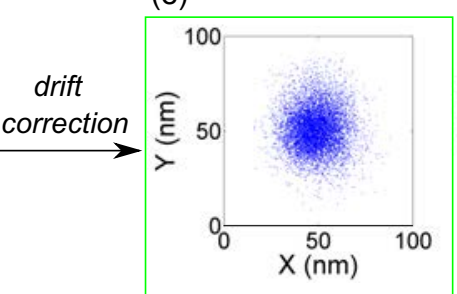

(f)

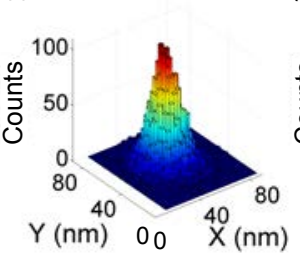

(g)

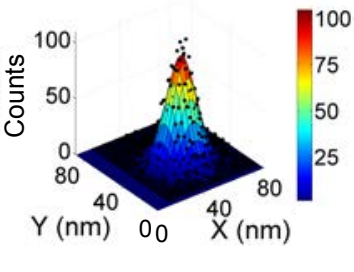

Figure 32: Lateral drift correction and single molecule localization. (a) Localizations of Atto647N molecules in the full-length data of movie 2, determined using rapidSTORM software. (b) Averaged sample drift calculated from the frame-to-frame displacements of all molecules. (c) Drift-corrected localizations after subtracting the averaged drift. (d) Zoom-in on the red square in (a), displaying a single molecule trace due to the sample drift from the original molecule positions. (e) Zoom-in on the green square in (c), showing drift-corrected localizations forming a well-defined cluster. (f) 2D histogram of the localizations in (e). (g) 2D Gaussian fit to the histogram in (f). Data analysis assisted by Simon Stein. 
(a)
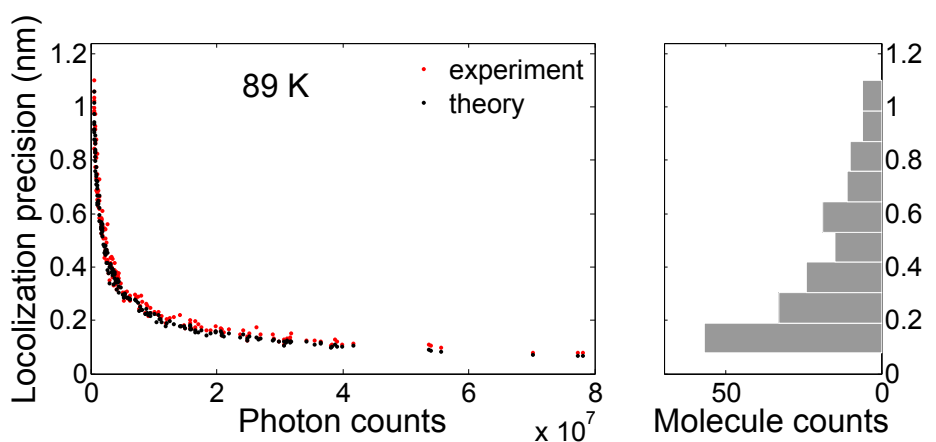

(b)

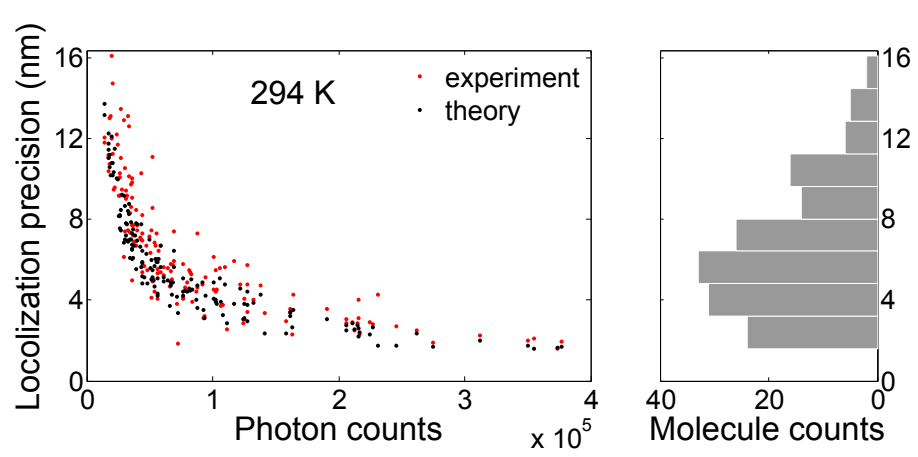

Figure 33: Comparison of localization precision between $89 \mathrm{~K}$ and $294 \mathrm{~K}$. The determined localization precision at $89 \mathrm{~K}$ (a) and $294 \mathrm{~K}$ (b) was plotted against the photon counts detected from each single molecule. The theoretically expected values ${ }^{[73}$ were computed separately for each molecule to include the influence of the slightly different local background. The number of molecules was histogrammed with respect to the localization precision. Data analysis assisted by Simon Stein.

\section{Dual Molecule Colocalization}

\subsection{Introduction}

Most super-resolution microscopy techniques at RT are applied to densely labeled samples, and deliver a resolution of several tens of nanometers. Another important application of "super-resolution" is to determine the distance between just two molecules on the nanometer scale. This is important e.g. to measure intramolecular distances, thus could contribute greatly to structural biology. One technique to measure nanometer distances between two fluorescent molecules is Förster (fluorescence) resonance energy transfer (FRET) ${ }^{74}$. However, this technique has a very limited accuracy, because the exact orientations of the two molecules are required to calculate the absolute distance ${ }^{[75}$, and this is very hard to measure in solution for freely rotating dye molecules.

Cryo-FM provides alternative possibilities to measure distances between two fluorescent 
molecules. The drastically enhanced photostability at CT enables single molecule localization with sub-nanometer precision. The slowed-down blinking and the fixed orientations of fluorophores provide new ways to colocalize two molecules with single nanometer resolution.

Recently, Weisenburger et al. demonstrated colocalization of two fluorophores on a DNA strand with single nanometer resolution $\underline{48}$. They measured intensity traces of the doubledye labeled DNA, and due to the extended dark state of organic dyes at CT, they could sort the cases when both dye molecules were on, and when only one molecule was on. By performing localizations for these two cases separately, they determined single molecule positions and averaged double-molecule positions, and then back-calculated the distance between the two molecules. However, as discussed in their report, this approach has a very limited success rate (useful intensity traces over all intensity traces) of about $0.1 \%$. This is the case because the intensity-based analysis suffers from the irregular blinking of the two molecules. Therefore, this approach can be hardly generalized due to the poor reproducibility and the low throughput.

In this section, several other approaches are proposed and examined. Although no robust solution has been yet fully established, the limitations of each approach and the possible solutions are discussed. This aims at providing a reference point for follow-up work, and at stimulating future investigations in this field.

\subsection{Colocalization by separate emission}

To separate two fluorescent molecules located within the diffraction barrier, one straightforward approach is to use two fluorophores with well separated emission spectra. These two molecules can be imaged through different color channels, and can be localized with nanometer precision in each channel. Organic dyes provide many options for fluorophore pairs without spectral crosstalk. For example, Atto488 and Atto674N can be exited individually at $488 \mathrm{~nm}$ and $637 \mathrm{~nm}$, and can be imaged through two spectral channels separated by over $150 \mathrm{~nm}$ (Fig. 34).

The main challenge for this approach is to correct the position offset created by the chromatic aberration between the two color channels. Typically, the emission spectra of the selected dye pair need to differ by over $100 \mathrm{~nm}$ to assure minimal spectral crosstalk. The resulting chromatic aberration creates position shifts that have to be taken into account when performing super-resolution microscopy on the nanometer scale ${ }^{76}$. Although it has been demonstrated that the chromatic offset can be corrected with nanometer precision $\underset{77}{ }$, 
the correction method is complicated and has limited reproducibility.

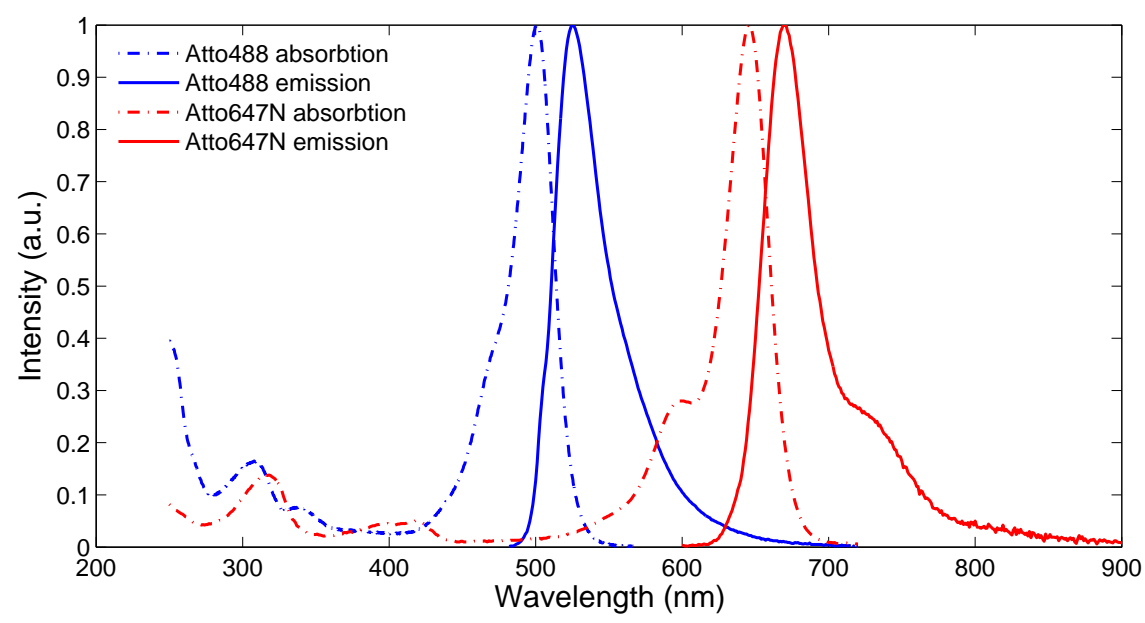

Figure 34: Spectra of Atto488 and Atto674N dyes. Well separated spectra of Atto488 and Atto674N dyes enable individual excitation and detection without spectral crosstalk.

In this project, when using a low NA air objective to image the sample through a thick optical window, the chromatic offset becomes more severe. As demonstrated in Fig. 35 , multi-color fluorescent beads were imaged in a green channel $(573 \mathrm{~nm}-613 \mathrm{~nm})$ and a red channel $(660 \mathrm{~nm}-710 \mathrm{~nm})$. The uniform distribution of the fluorophores inside the $100 \mathrm{~nm}$ bead assures an identical emission center for the two channels. However, the measured color centers differed by up to $100 \mathrm{~nm}$. The position offsets displayed an irregular and discontinuous profile across the FOV, which can be hardly corrected. Moreover, the offset profile differed from FOV to FOV in the same sample, which makes a general correction calibration of the imaging system hopeless.

In conclusion, it is very difficult to achieve a reliable, nanometer-accurate correction for the chromatic offsets when imaging through a thick optical window using a low NA objective. As a result, colocalizing two molecules by different emission colors is in practice hardly possible.

\subsection{Colocalization by individual excitation}

One way to circumvent the chromatic offset is to use two fluorophores that emit in the same spectral range, but can be individually excited. This approach can be realized by using long Stokes shift (LSS) dyes, which have an extra large Stokes shift of up to $\sim 200 \mathrm{~nm}$. If using one LSS dye together with a normal dye with similar emission spectrum, these two molecules can be excited separately at different wavelengths, but emit in the same spectral window without chromatic offset. An example of one possible dye combination is Atto647N 


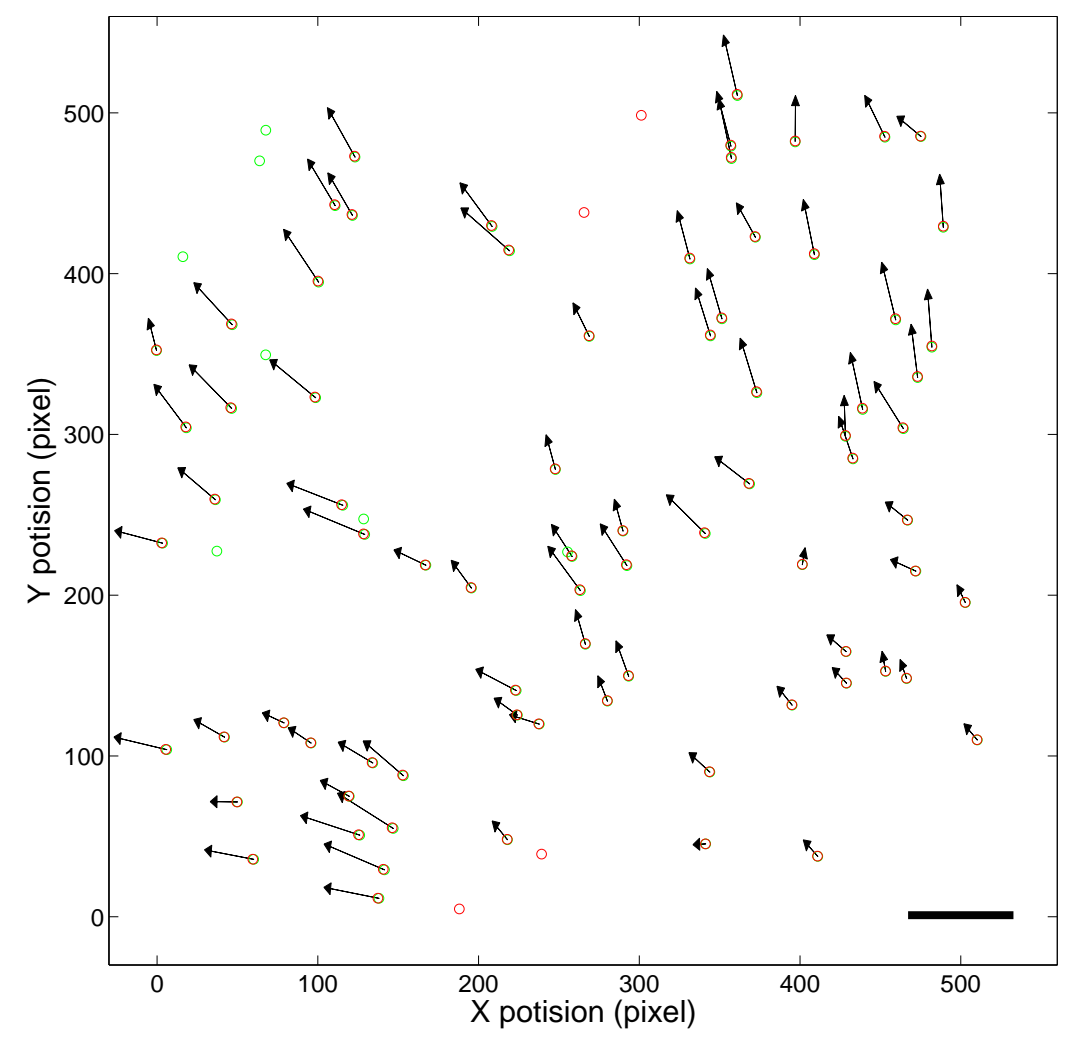

Figure 35: Chromatic offset of the cryo-imaging system. Multi-color fluorescent beads of $100 \mathrm{~nm}$ diameter (TetraSpeck, Thermo Fisher Scientific Inc.) were prepared on a silica coverslip, and the sample was imaged in the cryostat. Images were take alternatingly in a green channel (excitation $532 \mathrm{~nm}$, emission window 593-40) and in a red channel (excitation $637 \mathrm{~nm}$, emission window 685-50) by automatically switching the lasers and the emission filters using LabVIEW software. In total 6979 frames were acquired for each channel with $0.1 \mathrm{~s}$ exposure time and 100 EM-gain. The two channels were drift-corrected separately, and the beads were localized with a precision below $1 \mathrm{~nm}$ in each channel (method see section 9). The determined bead positions were marked as green and red circles for the green and red channels, respectively. The position offset between the two channels were visualized using arrows with enlarged lengths, corresponding to the scale bar of $100 \mathrm{~nm}$ in the plot. 
and Atto490LS, as shown in Fig. 36 .

One problem associated with LSS dyes is their low brightness as compared to normal organic dyes. As shown in table 1, in comparison to Atto647N, Atto490LS has a $\sim 4$ times lower extinction coefficient, $\mathrm{a} \sim 2$ times lower quantum yield, but a $\sim 1.4$ times shorter life-time. In total, this delivers an about 6 times lower brightness. As a result, when imaging with a 0.7 NA objective, single molecules of LSS dyes cannot be detected due to the poor $\mathrm{s} / \mathrm{n}$ ratio.

One way to increase the $\mathrm{s} / \mathrm{n}$ ratio is to use cryostats with an integrated high NA objective. These cryostats are being developed in several research groups (not published yet), and will become available in the near future. These systems use integrated objectives inside the cryostat with special cryo-compatible immersion medium, which increases the NA to over 1.2 , thus have the potential to resolve single molecules of LSS dyes.

Alternatively, new fluorescent markers are constantly being developed in research and in industry. For example, the newly discovered carbon nano particles (CNPs) have very broad excitation spectra and high brightness ${ }^{[78}$, which might be suitable for this approach.

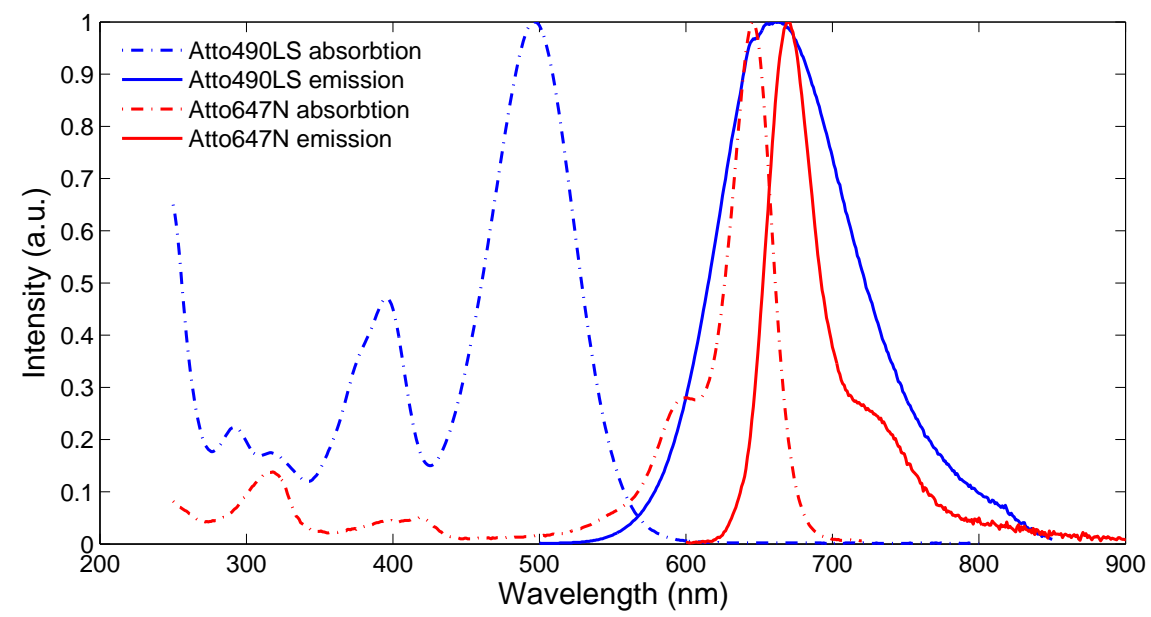

Figure 36: Spectra of Atto647N and Atto490LS dyes. Normal dye Atto647N and Long Stokes shift dye Atto490LS emit in the same spectral window of e.g. 685-50, but can be individually excited at $488 \mathrm{~nm}$ and $637 \mathrm{~nm}$, respectively.

\begin{tabular}{l|c|c|c} 
& extinction coeff. & quantum yield & fluorescence life-time \\
\hline Atto647N & $\varepsilon_{\max }=1.5 \times 10^{5} \mathrm{lmol}^{-1} \mathrm{~cm}^{-1}$ & $\eta_{f l}=60 \%$ & $\tau_{f l}=3.5 \mathrm{~ns}$ \\
\hline Atto490LS & $\varepsilon_{\max }=4.0 \times 10^{4} \mathrm{lmol}^{-1} \mathrm{~cm}^{-1}$ & $\eta_{f l}=30 \%$ & $\tau_{f l}=2.6 \mathrm{~ns}$
\end{tabular}

Table 1: Property comparison between Atto647N and Atto490LS dyes. Data from www.attotec.com. 


\subsection{Colocalization by blinking}

The slowed-down blinking of organic dyes at CT provides new possibilities to colocalize two molecules below the diffraction limit of optical microscopy. As discussed in section 7.2 , Alexa647, Atto647N, and Cy5 dyes blink with an average on/off ratio of less than 0.5 at $89 \mathrm{~K}$. This means that when imaging two of these molecules with an appropriate exposure time, only one molecule will be captured in each frame. Thus, the two molecules can be separated in the temporal domain. This situation can be considered as a special case of SMLM, in which the on/off ratio is tuned to a much lower value to image densely labeled samples. In general, $N$ molecules in the diffraction limited area can be separated in time if the on/off ratio is $1 / N$.

After temporally separating the two molecules, the next challenge is to assign the photons in each frame to either of the molecules to achieve high localization precision. In general, the localization precision of SMLM is determined by the total number of photons that can be detected from each single molecule. In the case of SMLM of densely labeled samples at $\mathrm{RT}$, as the photon assignment is difficult due to the dense labeling, the localization precision is typically determined by the photon number captured in each frame, which results in a resolution of several tens of nanometers. In the case of cryo-FM of just two molecules, each molecule can emit in total millions of photons before bleaching. But these photons are unevenly distributed in different frames by the alternating on and off switching of the two molecules. Therefore, to achieve a high localization precision, the photons in each frame have to be assigned to either of the molecules. Then, the accumulated millions of photons can deliver the position of each molecule with sub-nanometer precision. From these positions, the distance between the two molecules can be determined with single nanometer resolution. In short, photon assignment is the key for this approach.

There are various possibilities to identify the molecule in each frame. For example, the dipole orientation of a fluorophore is fixed after freezing, and the chance for two molecules to have exactly the same orientation is almost zero. Therefore, their defocused patterns differ from each other ${ }^{79}$, which can be used to differentiate between the two molecules. Another possible method is to choose two dyes with similar emission range but different fluorescence life-times, e.g. Atto647N (3.5 ns) and Cy5 (1 ns), and measure the life-time simultaneously with imaging by using fluorescence life-time imaging microscopy (FLIM).

However, these approaches are feasible only by using high NA objectives, which deliver sufficient $\mathrm{s} / \mathrm{n}$ ratio and photo counts per frame. For the cryostat equipped with a $0.7 \mathrm{NA}$ 
objective, these methods are difficult to implement due to the poor s/n ratio when imaging with short exposure time to capture the blinking. But when high NA cryo-imaging systems become available in the future, these approaches can be employed to colocalize two fluorescent molecules at CT with single nanometer resolution.

\subsection{Colocalization by polarization}

\subsubsection{Theory}

At CT, the dipole orientation of fluorophores is fixed after freezing. This provides another possibility of switching on and off single molecules by using linearly polarized excitation. The excitation efficiency is determined by the relative angle $\alpha_{r e l}$ between the dipole orientation of the fluorophore and the polarization direction of the excitation light. By changing the polarization direction, the fluorescence intensity $I$ can be continuously modulated as $I \propto$ $\cos ^{2}\left(\alpha_{r e l}\right)$.

For two molecules located within one diffraction-limited spot, the chance that they have exactly the same orientation is almost zero. Thus, there exist two excitation directions in which only one molecule can be excited, i.e. the directions perpendicular to the dipole orientation of one molecule. This creates the possibility of individual "on-switching", which can be used to colocalize two molecules within the diffraction barrier as described in previous sections. This method requires the prior knowledge of the orientations of the two molecules, which can be determined as described below.

In fact, one of the two molecules does not have to be completely turned off to perform colocalization. If the molecular brightness and the dipole orientation are known, the fluorescence intensity of each molecule can be determined at arbitrary excitation directions. The resulting two PSFs with different amplitudes and positions overlap, generating an image with a center position located between the two molecules. This position can be measured experimentally from the center of mass $\vec{r}_{C M}$ of the superimposed image, which is given as a weighted average of the form

$$
\vec{r}_{C M}=\frac{I_{1} \vec{r}_{1}+I_{2} \vec{r}_{2}}{I_{1}+I_{2}}
$$

The intensities of the two molecules $I_{1}$ and $I_{2}$ are defined by their intrinsic brightness and dipole orientations, which can also be measured using the method described below. Then, 


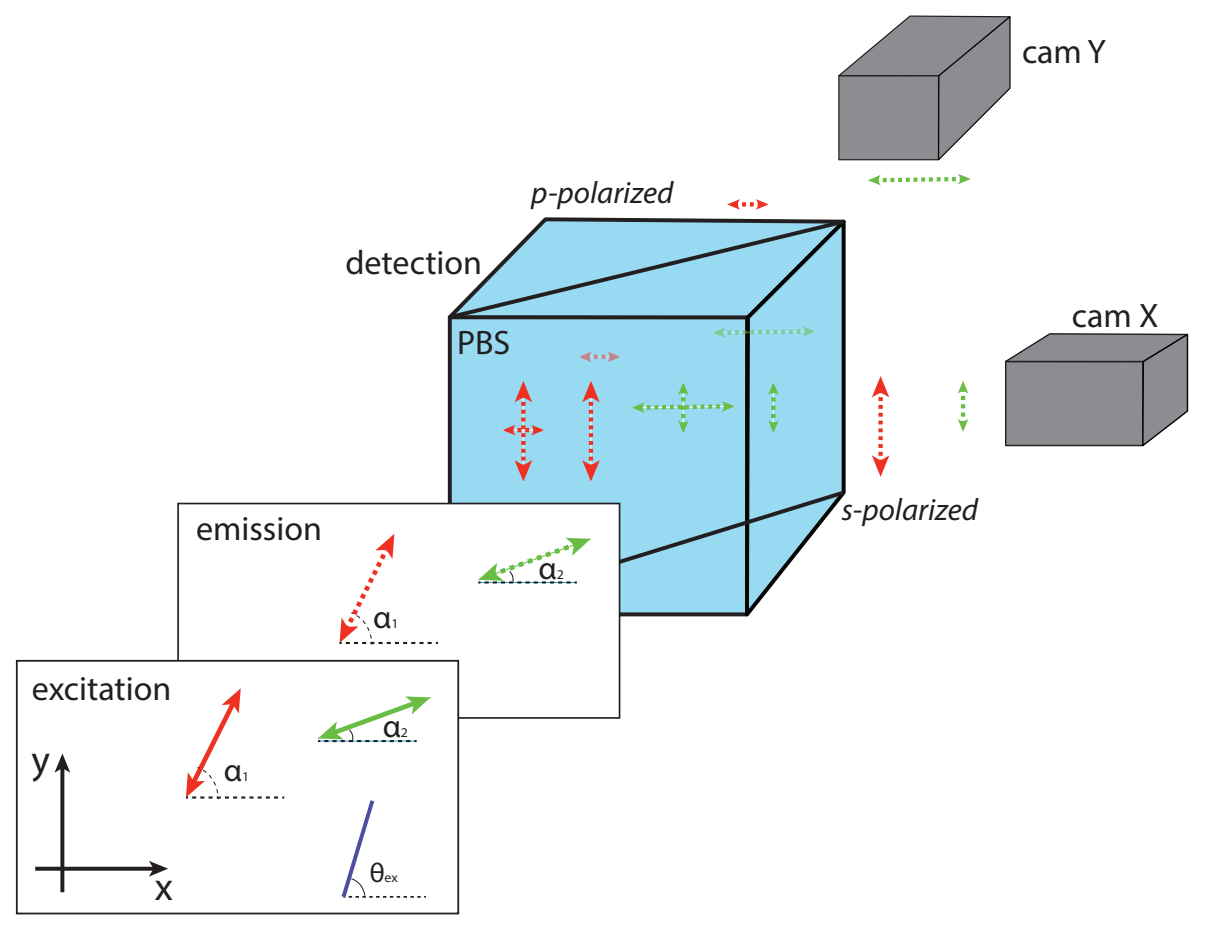

Figure 37: Schematic of linearly polarized excitation and detection of two molecules.

the positions of the two molecules $\vec{r}_{1}$ and $\vec{r}_{2}$ can be calculated, from which the distance between them can be determined.

To measure the brightness and the dipole orientation of two molecules located in one diffraction-limited spot, one method was developed in this project using linearly polarized excitation and detection. As shown in Fig. 37, assume that the two molecules have fluorescence dipoles oriented at angle $\alpha_{1}$ and $\alpha_{2}$ in the sample plane, and that the excitation light is linearly polarized at angle $\theta_{e x}$. Then the fluorescence intensities of the two molecules $I_{1}$ and $I_{2}$ are expressed as:

$$
\begin{aligned}
& I_{1}=k_{1} \cos ^{2}\left(\theta_{e x}-\alpha_{1}\right) \\
& I_{2}=k_{2} \cos ^{2}\left(\theta_{e x}-\alpha_{2}\right)
\end{aligned}
$$

where $k_{1}$ and $k_{2}$ summarize the molecular properties (e.g. extinction coefficient, quantum yield, life-time etc.) which influence the fluorophore brightness.

As the two molecules are located within one diffraction-limited spot, they will be registered as one molecule with intensity:

$$
I_{1+2}=k_{1} \cos ^{2}\left(\theta_{e x}-\alpha_{1}\right)+k_{2} \cos ^{2}\left(\theta_{e x}-\alpha_{2}\right)
$$


Using the identity

$$
\cos ^{2}(x)=\frac{(1+\cos 2 x)}{2}
$$

Eq. 6 can be rewritten as:

$$
\begin{aligned}
I_{1+2} & =k_{1} \frac{1+\cos \left[2\left(\theta_{e x}-\alpha_{1}\right)\right]}{2}+k_{2} \frac{1+\cos \left[2\left(\theta_{e x}-\alpha_{2}\right)\right]}{2} \\
& =\frac{k_{1}+k_{2}}{2}+\frac{k_{1}}{2} \cos \left(2 \theta_{e x}-2 \alpha_{1}\right)+\frac{k_{2}}{2} \cos \left(2 \theta_{e x}-2 \alpha_{2}\right) \\
& =B+A \cos \left(2 \theta_{e x}-\Phi\right)
\end{aligned}
$$

where

$$
\begin{aligned}
& B=\frac{k_{1}+k_{2}}{2} \\
& A=\sqrt{\frac{k_{1}^{2}+k_{2}^{2}}{4}+\frac{k_{1} k_{2}}{2} \cos \left[2\left(\alpha_{1}-\alpha_{2}\right)\right]} \\
& \Phi=\arctan \left[\frac{k_{1} \sin \left(2 \alpha_{1}\right)+k_{2} \sin \left(2 \alpha_{2}\right)}{k_{1} \cos \left(2 \alpha_{1}\right)+k_{2} \cos \left(2 \alpha_{2}\right)}\right]
\end{aligned}
$$

Eq.7 shows that the total intensity of the two molecules $I_{1+2}$ is defined by three independent parameters $B, A$, and $\Phi$, which can be experimentally determined in two ways. (i) Measuring $I_{1+2}$ at three different excitation angles, and solving the resulting equation set. (ii) Measuring $I_{1+2}$ at a sweep of excitation angles with small steps, and fitting the resulting curve with the three parameters.

However, these parameters are defined by four unknowns that describe the physical system, namely $k_{1}, k_{2}, \alpha_{1}$, and $\alpha_{2}$. Therefore, these four unknowns cannot be uniquely determined from the obtained three parameters. To make the system well-determined, one more piece of information has to be added. To do this, a polarizing beam splitter (PBS) is placed in the detection path. The PBS splits the emitted fluorescence in two orthogonal polarizations, thus creating more information. Assume that the fluorescence has the same polarization direction as the dipole orientation. Then, the fluorescence intensity $I_{x}$ and $I_{y}$ registered on camera $\mathrm{X}$ and $\mathrm{Y}$ can be expressed as:

$$
\begin{aligned}
& I_{x}=\underbrace{k_{1} \cos ^{2}\left(\theta_{e x}-\alpha_{1}\right) \cdot \cos ^{2}\left(\alpha_{1}\right)}_{I_{1 x}}+\underbrace{k_{2} \cos ^{2}\left(\theta_{e x}-\alpha_{2}\right) \cdot \cos ^{2}\left(\alpha_{2}\right)}_{I_{2 x}} \\
& I_{y}=\underbrace{k_{1} \cos ^{2}\left(\theta_{e x}-\alpha_{1}\right) \cdot \sin ^{2}\left(\alpha_{1}\right)}_{I_{1 y}}+\underbrace{k_{2} \cos ^{2}\left(\theta_{e x}-\alpha_{2}\right) \cdot \sin ^{2}\left(\alpha_{2}\right)}_{I_{2 y}}
\end{aligned}
$$


$I_{x}$ and $I_{y}$ can be transformed in analogy to Eq. 7 as:

$$
\begin{aligned}
I_{x} & =\underbrace{\left[k_{1} \cos ^{2}\left(\alpha_{1}\right)\right]}_{=: k_{1 x}} \cos ^{2}\left(\theta_{e x}-\alpha_{1}\right)+\underbrace{\left[k_{2} \cos ^{2}\left(\alpha_{2}\right)\right]}_{=: k_{2 x}} \cos ^{2}\left(\theta_{e x}-\alpha_{2}\right) \\
& =\frac{k_{1 x}+k_{2 x}}{2}+\frac{a_{1}}{2} \cos \left(2 \theta_{e x}-2 \alpha_{1}\right)+\frac{a_{2}}{2} \cos \left(2 \theta_{e x}-2 \alpha_{2}\right) \\
& =B_{x}+A_{x} \cos \left(2 \theta_{e x}-\Phi_{x}\right) \\
I_{y} & =\underbrace{\left[k_{1} \sin ^{2}\left(\alpha_{1}\right)\right]}_{=: k_{1 y}} \cos ^{2}\left(\theta_{e x}-\alpha_{1}\right)+\underbrace{\left[k_{2} \sin ^{2}\left(\alpha_{2}\right)\right]}_{=: k_{2 y}} \cos ^{2}\left(\theta_{e x}-\alpha_{2}\right) \\
& =\frac{k_{1 y}+k_{2 y}}{2}+\frac{a_{1}}{2} \cos \left(2 \theta_{e x}-2 \alpha_{1}\right)+\frac{a_{2}}{2} \cos \left(2 \theta_{e x}-2 \alpha_{2}\right) \\
& =B_{y}+A_{y} \cos \left(2 \theta_{e x}-\Phi_{y}\right)
\end{aligned}
$$

The six parameters $B_{x}, A_{x}, \Phi_{x}, B_{y}, A_{y}, \Phi_{y}$ can be experimentally determined as described before. From these six parameters, the four physical unknowns $k_{1}, k_{2}, \alpha_{1}$, and $\alpha_{2}$ can be calculated by solving the equations $8[9][0$. Thus, the brightness and the orientation of the two molecules can be determined.

This theory was developed by Simon Stein.

\subsubsection{Experimental setup}

The experimental setup is shown in Fig. 38. To realize a linearly polarized excitation at different angles, a half-wave plate is placed in the excitation path to alter the polarization direction of the laser. The half-wave plate is mounted in a motorized rotary stage, which is controlled using a custom-written LabVIEW software. When rotating the half-wave plate by angle $\theta$, the polarization direction of the laser is changed by $2 \theta$, but the excitation intensity distribution in the sample plane remains unchanged. This is crucial for the intensity-based analysis.

To perform polarized detection, a PBS cube is mounted after the tube lens. The PBS transmits the p-polarized light and reflects the s-polarized light, which are imaged separately on two cameras arranged perpendicular to each other. Introducing the PBS after the tube lens (instead of using two tube lenses after the PBS) makes the alignment of the two cameras easier and more accurate. However, this also changes the original magnification by $\sim 3 \%$, because the PBS cube delays the image light. To correct for this, a calibration grid with known dimensions are used to adjust the magnification for each camera. 


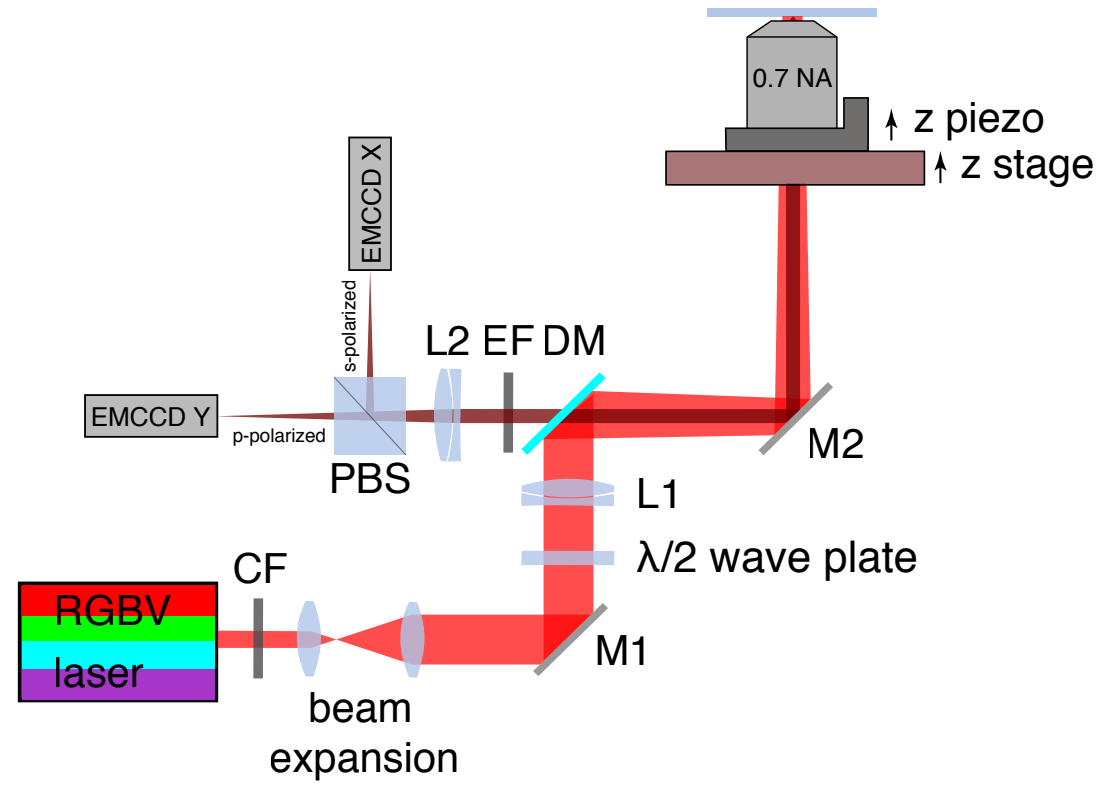

(a)

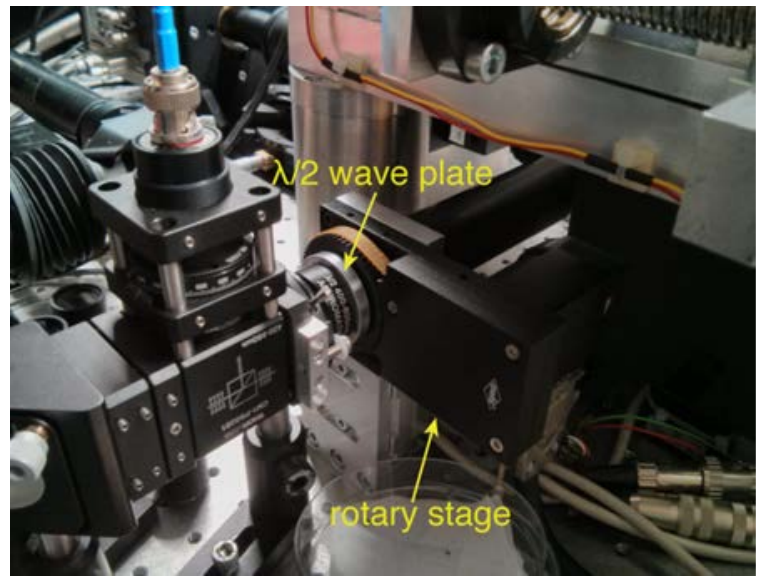

(b)

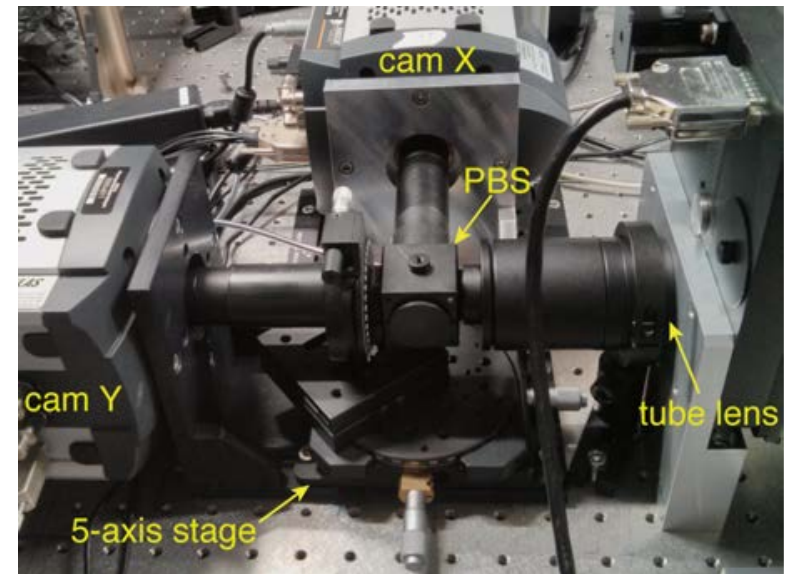

(c)

Figure 38: Experimental setup for polarization microscopy. (a) Schematic of the optical arrangement. On top of the existing fluorescence microscope as described in Fig. 5, a half-wave plate is inserted into the excitation path to change the polarization direction of the laser. In the detection path, a PBS cube is placed after the tube lens (L2) to split the s- and p-polarized emission onto two EMCCD cameras. (b) A photo of the excitation path. (c) A photo of the the detection path.

As shown in Fig. 39, a $10 \mu \mathrm{m}$ grid was imaged on both cameras. The size of the grid image was measured according to the pixel size of the camera $(16 \mu \mathrm{m} / \mathrm{pixel})$. The measured grid size was matched to its physical size under the magnification of the objective by shifting the camera along the optical axis. After adjusting the magnification of both cameras to the same value, the overlap of the grid images displayed only a residual shift/rotation (Fig. $39 \mathrm{c}$ ). This manual alignment has a precision of about two pixels, which is $\sim 0.4 \%$ for a camera 
with $512 \times 512$ pixels.

To further align the two cameras with respect to image shift and rotation, fluorescent beads were used as markers, and were imaged on the two cameras simultaneously (Fig.40). The bead image on camera $\mathrm{X}$ was matched to the image on camera $\mathrm{Y}$ by manually adjusting the position/rotation of the PBS cube using a 5-axis stage (altering the PBS does not change bead image on camera Y). This "rough overlapping" is aimed at an easier identification of the same molecule on the two cameras in the post-analysis. As only the intensity of the molecule is used for the analysis, the accuracy of the overlap is not critical. The accuracy of the manual alignment and the visual judgment is sufficient for this purpose. The remaining residue offsets between the two images can be tolerated by the analysis routine.

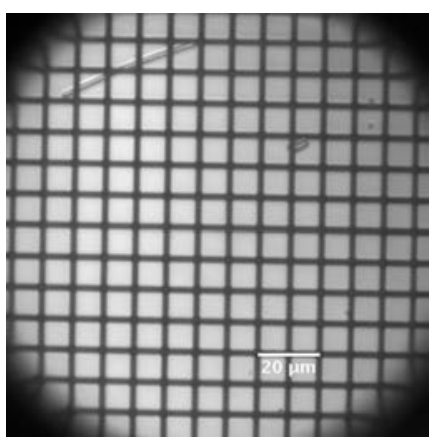

(a) Cam X

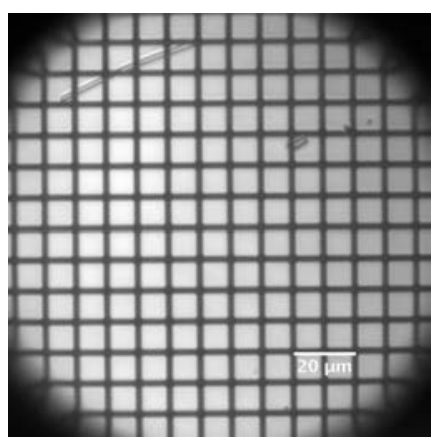

(b) Cam Y

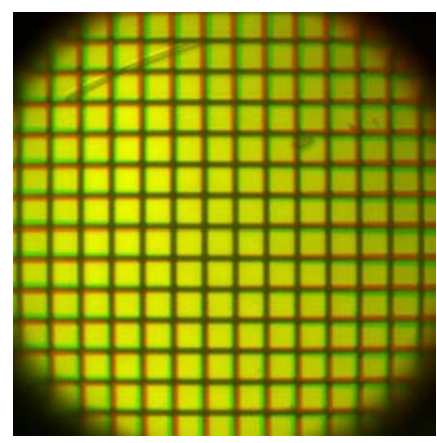

(c) Merge

Figure 39: Camera magnification adjustment. (a) Image of a $10 \mu \mathrm{m}$ calibration grid (R1L3S3P, Thorlabs GmbH, Germany) captured on camera X. Distance measured by the camera pixel size under $60 \times$ magnification of the objective. (b) Image of the grid captured on camera Y. (c) Overlap of the two images.

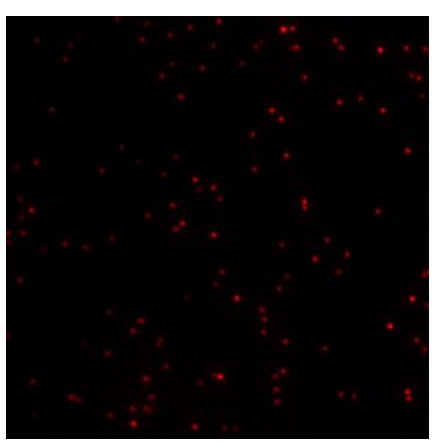

(a) Cam X

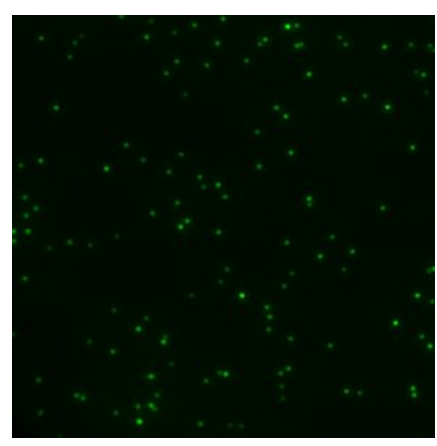

(b) Cam Y

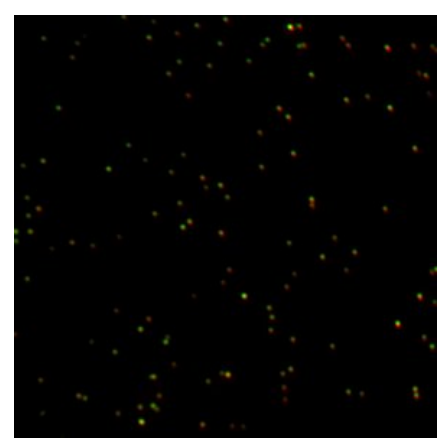

(c) Merge

Figure 40: Camera alignment. (a) Image of fluorescent beads (TetraSpeck, Thermo Fisher Scientific Inc. ) captured on camera X. (b) Image of the beads captured on camera Y. (c) Merge of the two images. 


\subsubsection{System calibration}

When imaging an empty coverslip with rotating linearly polarized excitation, the background captured on the two cameras also oscillates. As shown in Fig.41, the averaged background intensity varies correspondingly to the polarization direction of the excitation light. The oscillating signal of the $\mathrm{X}$ and $\mathrm{Y}$ cameras are out of phase, indicating that the background is (mainly) linearly polarized. This is because the background sources in this setup - the fluorescent impurities in the objective lenses and the adhesives - have fixed orientations, and the reflected laser from the lenses and the coverslip sustains the linear polarization.

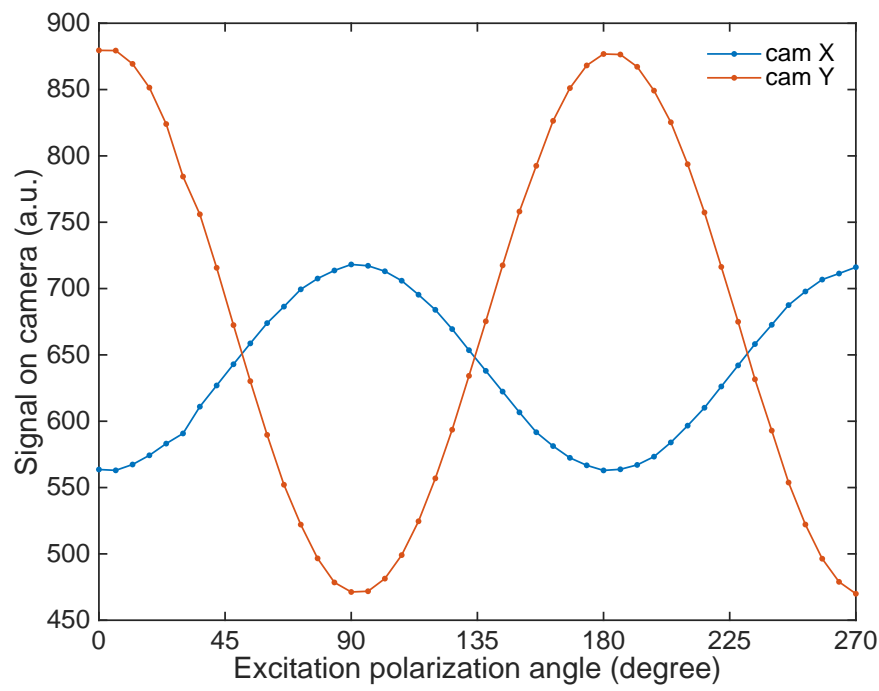

Figure 41: Background fluctuation in polarization microscopy. The half-wave plate in the excitation path was rotated at a step size of $3^{\circ}$, which changed the polarization direction of the excitation laser at a step size of $6^{\circ}$. A silica coverslip was illuminated by this linearly polarized light, and was imaged through a PBS onto two cameras with $5 \mathrm{~s}$ exposure time. The average background intensity captured on the two cameras was plotted against the angle of the excitation polarization.

The amplitude of the background fluctuation differs by $\sim 2.7$ times between the two cameras. This is because the optical components such as the PBS, the dichroic beam splitter, and the dielectric mirrors have different transmission and reflection efficiency for the s- and ppolarized light. For example, at an angle of incidence (AOI) of $45^{\circ}$, the transmission efficiency of the dichroic beam splitter (FF410/504/582/669, Semrock, USA) differs by $\sim 10 \%$ between the s- and p-polarized light. Moreover, these optical elements also introduce a slight phase shift between the s- and p-waves if the AOI is not zero. This makes the linearly polarized excitation and emission slightly elliptically polarized. As the ellipticity of the polarization is rather small, this effect can be neglected in the experiment. 
All these effects are encoded in the background fluctuation with respect to the polarization direction of the excitation light, which is used as a reference for calibration. In the experiment, the local background for each molecule is measured, and is subtracted from the detected intensity to obtain the actual molecule brightness.

\subsubsection{Single molecule polarization microscopy}

To validate the intensity modulation of single molecules using polarized excitation and detection, Atto647N molecules were imaged in the polarization microscope at $89 \mathrm{~K}$. The dye solution was deposited onto a silica coverslip using a pipette and was air-dried to create randomized fluorophore orientations. The sample was excited by a linearly polarized laser with changing polarization direction at a step size of $6^{\circ}$. The emitted fluorescence was split into two orthogonal polarization directions using a PBS cube, and was imaged onto two cameras. Images were taken with a relative long exposure time of $5 \mathrm{~s}$ to average out the single molecule blinking, and to increase the $\mathrm{s} / \mathrm{n}$ ratio.

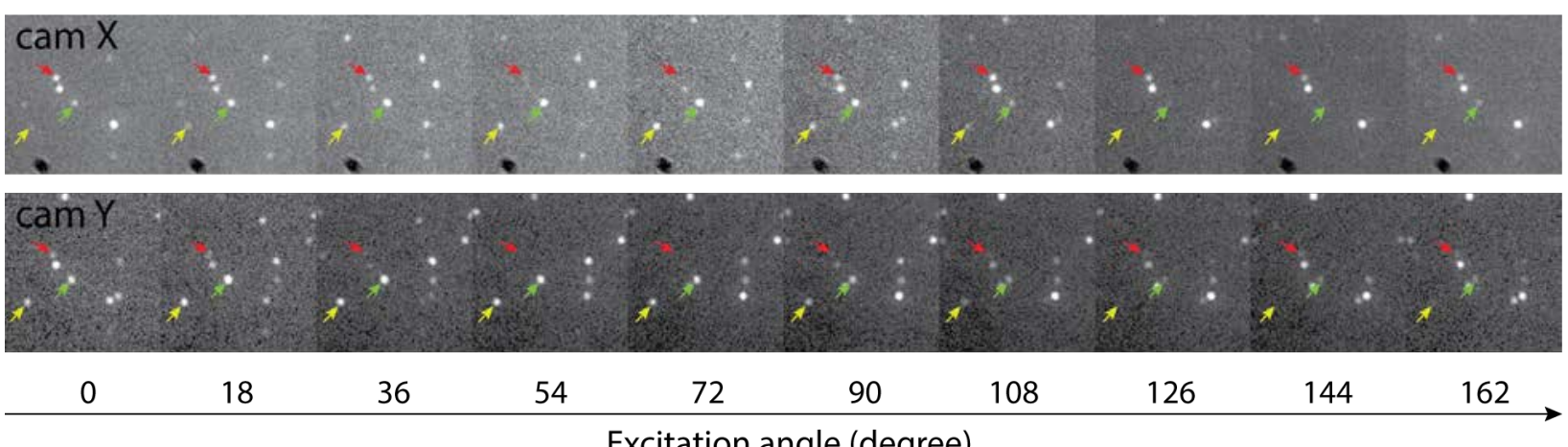

Excitation angle (degree)

Figure 42: Polarized excitation and detection of single molecules. Atto647N molecules were excited by linearly polarized laser at different angles at $89 \mathrm{~K}$, and were imaged through a PBS onto two cameras. Images were taken with $5 \mathrm{~s}$ exposure time. Three molecules with different modulation phases were highlighted by arrows.

As shown in movie 9 (extracted frames in Fig.42), the intensity of the single molecules is periodically modulated by the rotating linear excitation. The modulation phase is different from molecule to molecule, indicating that the molecules are in different orientations on the coverslip. For each molecule, the intensities captured on the X and Y cameras oscillate in phase, indicating that the polarization direction of fluorescence emission is fixed, and is uncorrelated to of the excitation polarization direction. The smooth intensity change 
suggests that the blinking is averaged out during the $5 \mathrm{~s}$ exposure time, which is advantageous for an accurate intensity-based analysis. The high photostability of fluorophores at $89 \mathrm{~K}$ enables long measurements of single molecules till a desired precision is reached.

These results demonstrate the feasibility of modulating the excitation of single molecules with fixed dipole orientation using linearly polarized excitation at different angles. The intensity modulation is continuous with complete "off" states. This offers another possibility of switching single molecule fluorescence, and can be used for the colocalization of two molecules with single nanometer resolution at CT.

\subsubsection{Outlook}

The preliminary results in this section demonstrate that polarization microscopy can be used to control the single molecule excitation in a continuous manner at $89 \mathrm{~K}$. The high photostability of fluorophores at CT enables the measurement of single molecule orientation with very high precision. The current challenge of testing the proposed dual molecule colocalization lies in the preparation of samples labeled with two fluorophores at a known distance on the nanometer scale. Although techniques such as DNA origami ${ }^{80}$ provide the possibility of designing custom-defined "nano-rulers", labeling the sample with just two fluorophores with high efficiency $(>90 \%)$ is still challenging.

Moreover, the purification of the sample also plays an important role in cryo-FM. In the FOV, there are only about 200-400 molecules, and the measurement time of each FOV extends to several hours to obtain single nanometer precision. Therefore, the amount of free dyes molecules and fluorescent impurities needs to be minimized to achieve high throughput and reasonable statistics. 


\section{CONCLUSION \& OUTLOOK}

This work was concerned with the development and testing of a new cryo-fluorescence microscopy system, which can be employed for single molecule microscopy at $89 \mathrm{~K}$ and CLEM experiments. This system features excellent thermal and mechanical stability, superior single molecule imaging quality, intact sample transfer at CT, and additional gas supply to the sample.

Using this system, it is found that the photostability of organic dyes (such as Atto647N, Alexa647, Atto655, Cy5, etc.) can be boosted by two orders of magnitude when cooling down to $89 \mathrm{~K}$. The resulting millions of detectable photons per molecule allow for single molecule localization with sub-nanometer precision. Moreover, the blinking of Alexa647, Atto647N, and Cy5 molecules is slowed down by a factor of ten at $89 \mathrm{~K}$, which results in an on/off ratio below 0.5. This provides the possibility to separate two molecules within one diffraction-limited spot temporally. In contrast, Atto488 and Alexa488 molecules dive into drastically extended dark states in the absence of oxygen (in vacuum) at $89 \mathrm{~K}$, and return to the fluorescent state stochastically upon laser irradiation at $473 \mathrm{~nm}$. For these dyes, the vacuum cryostat automatically creates the condition for STORM, which makes cryo-STORM directly applicable.

This cryo-imaging system provides also the possibility to measure distances between two fluorescent molecules on the single nanometer scale. Current bottlenecks are the chromatic offset, the poor s/n ratio, and the difficult photon assignment. In the future, $t$, these limitations can be overcome by the development of brighter dyes and high NA cryostats with integrated objectives, which will make many microscopy techniques such as defocused imaging, FLIM, etc. applicable at CT for super-resolution dual-molecule colocalization. Polarization microscopy provides another possibility to circumvent these limitations. The fluorescence intensity of single molecules can be contentiously modulated using linearly polarized excitation. When combined with polarized detection, the molecule brightness and orientation can also be determined. This molecular information can be directly employed to determine the distance between two molecules on the nanometer length scale. 


\section{APPENDIX}

\section{Coverslip Cleaning Protocol}

\section{MATERIAL:}

1. Menzel coverslips

2. Teflon coverslip holder

3. Washing buffer: $10 \mathrm{~g} \mathrm{KOH}+50 \mathrm{ml} \mathrm{H} 2 \mathrm{O}+230 \mathrm{ml}$ ethanol

4. D-water

5. Incubator

\section{PROCEDURE:}

1. Place the coverslips in the Teflon holder.

2. Ultrasonicate the coverslips in the washing buffer for $10 \mathrm{~min}$.

3. Discard the washing buffer into the container for waste liquid (do not pour it into the sink because of $\mathrm{KOH}$ !).

4. Ultrasonicate the coverslips in D-water for $3 \mathrm{~min}$, change water, repeat 3 times.

5. Dry the coverslips in the incubator at $70^{\circ} \mathrm{C}$ for two hours.

6. Plasma clean the coverslips for $\sim 15$ min (remove the metal handle from the Teflon holder). 


\section{Objective Photon Collection Efficiency}

This section derives the relationship between the photon collection efficiency of an objective and the numerical aperture (NA).

The objective photon collection efficiency is proportional to the volume of the solid angle $\Omega$ that can be captured by the objective. In spherical coordinate, $\Omega$ can be expressed using the polar angle $\theta$ and the azimuthal angle $\varphi$ as:

$$
d \Omega=\sin \theta d \theta d \varphi
$$

For an objective, $\varphi$ goes from 0 to $2 \pi$, and $\theta$ defines the NA of the objective as:

$$
N A=n \sin \theta
$$

where $n$ is the refractive index of the medium in which the objective is working.

The volume $V$ encapsulated by the solid angle $\Omega$ can be expressed as:

$$
\begin{aligned}
V & =\int d \Omega \\
& =\int_{0}^{2 \pi} \int_{0}^{\theta} \sin \theta^{\prime} d \theta^{\prime} d \varphi^{\prime} \\
& =\int_{0}^{2 \pi} d \varphi \int_{0}^{\theta} \sin \theta^{\prime} d \theta^{\prime} \\
& =2 \pi(1-\cos \theta) \\
& =2 \pi\left(1-\sqrt{1-\sin ^{2} \theta}\right)
\end{aligned}
$$

Substituting $\sin \theta$ by $N A / n$ (from equation 16 ) gives:

$$
V=2 \pi\left(1-\sqrt{1-(N A / n)^{2}}\right)
$$

The objective photon collection efficiency is proportional to $V$. 


\section{BIBLIOGRAPHY}

[1] Moerner, W. E. \& Kador, L. Optical detection and spectroscopy of single molecules in a solid. Phys. Rev. Lett. 62, 2535-2538 (1989).

[2] Orrit, M. \& Bernard, J. Single pentacene molecules detected by fluorescence excitation in a p-terphenyl crystal. Phys. Rev. Lett. 65, 2716-2719 (1990).

[3] Plakhotnik, T., Donley, E. A. \& Wild, U. P. Single-molecule spectroscopy. Annu. Rev. Phys. Chem. 48, 181-212 (1997).

[4] Güttler, F., Irngartinger, T., Plakhotnik, T., Renn, A. \& Wild, U. P. Fluorescence microscopy of single molecules. Chem. Phys. Lett. 217, 393-397 (1994).

[5] Moerner, W. E. \& Orrit, M. Illuminating single molecules in condensed matter. Science 283, 1670-1676 (1999).

[6] Weiss, S. Fluorescence spectroscopy of single biomolecules. Science 283, 1676-1683 (1999).

[7] Moerner, W. E. Single-molecule optical spectroscopy and imaging: from early steps to recent advances. In Single Molecule Spectroscopy in Chemistry, Physics and Biology, 25-60 (Springer, 2010).

[8] Moerner, W. E. Nobel lecture: Single-molecule spectroscopy, imaging, and photocontrol: Foundations for super-resolution microscopy. Angew. Chem. Int. Ed. 54, 8067-8093 (2015).

[9] Kozankiewicz, B. \& Orrit, M. Single-molecule photophysics, from cryogenic to ambient conditions. Chem. Soc. Rev. 43, 1029-1043 (2014).

[10] Ambrose, W. \& Moerner, W. Fluorescence spectroscopy and spectral diffusion of single impurity molecules in a crystal. Nature 349, 225-227 (1991).

[11] Basché, T. \& Moerner, W. Optical modification of a single impurity molecule in a solid. Nature 355, 335-337 (1992). 
[12] Dickson, R. M., Cubitt, A. B., Tsien, R. Y. \& Moerner, W. E. On/off blinking and switching behaviour of single molecules of green fluorescent protein. Nature 388, 355358 (1997).

[13] Plakhotnika, T., Moernerb, W., Irngartinger, T. \& Wild, U. P. Single molecule spectroscopy in shpol'skii matrices. CHIMIA 48, 31-32 (1994).

[14] Schmidt, T., Schütz, G., Baumgartner, W., Gruber, H. \& Schindler, H. Imaging of single molecule diffusion. PNAS 93, 2926-2929 (1996).

[15] Betzig, E. \& Trautman, J. K. Near-field optics: Microscopy, spectroscopy, and surface modification beyond the diffraction limit. Science 257, 189-195 (1992).

[16] Betzig, E., Chichester, R., Lanni, F. \& Taylor, D. Near-field fluorescence imaging of cytoskeletal actin. Bioimaging 1, 129-135 (1993).

[17] Betzig, E. \& Chichester, R. J. Single molecules observed by near-field scanning optical microscopy. Science 262, 1422-1425 (1993).

[18] Ambrose, W. P., Goodwin, P. M., Martin, J. C. \& Keller, R. A. Single molecule detection and photochemistry on a surface using near-field optical excitation. Phys Rev Lett. 72, 160-163 (1994).

[19] Abbe, E. Beiträge zur Theori des Mikroskops und der Mikroskopischen Wahrnehmung. Archiv für mikroskopische Anatomy 9, 413-418 (1873).

[20] Rayleigh, L. On the theory of optical images, with special reference to microscopy. Philos. Mag. 42, 167-195 (1896).

[21] Sparrow, C. M. On spectroscopic resolving power. Astrophys. J. 44, 76-87 (1916).

[22] Cremer, C. \& Cremer, T. Considerations on a laser-scanning-microscope with high resolution and depth of field. Microsc. Acta. 81, 31-44 (1978).

[23] Sheppard, C. J. \& Wilson, T. The theory of the direct-view confocal microscope. J. Microsc. 124, 107-17 (1981).

[24] Müller, C. B. \& Enderlein, J. Image scanning microscopy. Phys. Rev. Lett. 104, id. 198101 (2010).

[25] Schulz, O. et al. Resolution doubling in fluorescence microscopy with confocal spinningdisk image scanning microscopy. PNAS 110, 21000-21005 (2013). 
[26] York, A. G. et al. Resolution doubling in live, multicellular organisms via multifocal structured illumination microscopy. Nat. Methods 9, 749-754 (2012).

[27] De Luca, G. M. et al. Re-scan confocal microscopy: scanning twice for better resolution. Opt. Express 4, 2644-2656 (2013).

[28] Gustafsson, M. G. L. Surpassing the lateral resolution limit by a factor of two using structured illumination microscopy. J. Microsc. 198, 82-87 (2000).

[29] Hell, S. \& Stelzer, E. H. Fundamental improvement of resolution with a 4pi-confocal fluorescence microscope using two-photon excitation. Opt. Commun. 93, 277-282 (1992).

[30] Gustafsson, M. G. L., Agard, D. A. \& Sedat, J. W. Sevenfold improvement of axial resolution in 3D wide-field microscopy using two objective lenses. Proc. SPIE $\mathbf{2 4 1 2}$, 147-156 (1995).

[31] Gustafsson, M. G. L., Agard, D. A. \& Sedat, J. W. I5M: 3D widefield light microscopy with better than 100nm axial resolution. J. Microsc. 195, 10-16 (1999).

[32] Huang, B., Babcock, H. \& Zhuang, X. Breaking the diffraction barrier: super-resolution imaging of cells. Cell 143, 1047-1058 (2010).

[33] Leung, B. O. \& Chou, K. C. Review of super-resolution fluorescence microscopy for biology. Appl. Spectrosc. 65, 967-980 (2011).

[34] Sahl, S. J. \& Moerner, W. E. Super-resolution fluorescence imaging with single molecules. Curr. Opin. Chem. Struct. Biol. 23, 778-787 (2013).

[35] Betzig, E. Nobel lecture: Single molecules, cells, and super-resolution optics. Rev. Mod. Phys. 87, 1153-1168 (2015).

[36] Hell, S. W. Far-field optical nanoscopy. Science 316, 1153-1158 (2007).

[37] Huang, B., Bates, M. \& Zhuang, X. Super resolution fluorescence microscopy. Annu. Rev. Biochem. 78, 993 (2009).

[38] Hell, S. W. et al. The 2015 super-resolution microscopy roadmap. J. Phys. D: Appl. Phys. 48, id. 443001 (2015).

[39] Hell, S. W. \& Wichmann, J. Breaking the diffraction resolution limit by stimulated emission: stimulated-emission-depletion fluorescence microscopy. Opt. Lett. 19, 780782 (1994). 
[40] Klar, T. A. \& Hell, S. W. Subdiffraction resolution in far-field fluorescence microscopy. Opt. Lett. 24, 954-956 (1999).

[41] Betzig, E. et al. Imaging intracellular fluorescent proteins at nanometer resolution. Science 313, 1642-1645 (2006).

[42] Rust, M. J., Bates, M. \& Zhuang, X. Sub-diffraction-limit imaging by stochastic optical reconstruction microscopy (STORM). Nat. Methods 3, 793-796 (2006).

[43] Hess, S. T., Girirajan, T. P. \& Mason, M. D. Ultra-high resolution imaging by fluorescence photoactivation localization microscopy. Biophys. J. 91, 4258-4272 (2006).

[44] Zondervan, R., Kulzer, F., Kol'chenk, M. A. \& Orrit, M. Photobleaching of rhodamine 6G in poly(vinyl alcohol) at the ensemble and single-molecule levels. J. Phys. Chem. A 108, 1657-1665 (2004).

[45] Schwartz, C. L., Sarbash, V. I., Ataullakhanov, F. I., McIntosh, J. R. \& Nicastro, D. Cryo-fluorescence microscopy facilitates correlations between light and cryo-electron microscopy and reduces the rate of photobleaching. J. Microsc. 227, 98-109 (2007).

[46] Weisenburger, S., Jing, B., Renn, A. \& Sandoghdar, V. Cryogenic localization of single molecules with angstrom precision. Proc. SPIE 8815, id. 88150D (2013).

[47] Giske, A. Ph.D. thesis: Cryosted microscopy a new spectroscopic approach for improving the resolution of STED microscopy using low temperature. University of Heidelberg (2007).

[48] Weisenburger, S. et al. Cryogenic colocalization microscopy for nanometer-distance measurements. ChemPhysChem 15, 763-770 (2014).

[49] Kaufmann, R. et al. Super-resolution microscopy using standard fluorescent proteins in intact cells under cryo-conditions. Nano Lett. 14, 4171-4175 (2014).

[50] Hussels, M., Konrad, A. \& Brecht, M. Confocal sample-scanning microscope for singlemolecule spectroscopy and microscopy with fast sample exchange at cryogenic temperatures. Rev. Sci. Instrum. 83, id. 123706 (2012).

[51] Hirschfeld, V., Paulsen, H. \& Hübner, C. G. The spectroscopic ruler revisited at 77 K. Phys. Chem. Chem. Phys. 15, 17664-17671 (2013).

[52] Sartori, A. et al. Correlative microscopy: Bridging the gap between fluorescence light microscopy and cryo-electron tomography. J. Struct. Biol. 160, 135-145 (2007). 
[53] Briegel, A. et al. Correlated light and electron cryo-microscopy. Methods Enzymol. 481, 317-341 (2010).

[54] Liu, B. et al. Three-dimensional super-resolution protein localization correlated with vitrified cellular context. Sci. Rep. 5, id. 13017 (2015).

[55] Chang, Y.-W. et al. Correlated cryogenic photoactivated localization microscopy and cryo-electron tomography. Nat. Methods 11, 737-739 (2014).

[56] Strnad, M. et al. Correlative cryo-fluorescence and cryo-scanning electron microscopy as a straightforward tool to study host-pathogen interactions. Sci. Rep. 5, id. 18029 (2015).

[57] Bellare, J. R., Davis, H. T., Scriven, L. E. \& Talmon, Y. Controlled environment vitrification system: an improved sample preparation technique. J. Electron Microsc. 10, 87-111 (1988).

[58] McDonald, K. L. A review of high-pressure freezing preparation techniques for correlative light and electron microscopy of the same cells and tissues. J. Microsc. 235, 273-281 (2009).

[59] Dubochet, J. Cryo-EM-the first thirty years. J. Microsc. 245, 221-224 (2012).

[60] van Driel, L. F., Valentijn, J. A., Valentijn, K. M., Koning, R. I. \& Koster, A. J. Tools for correlative cryo-fluorescence microscopy and cryo-electron tomography applied to whole mitochondria in human endothelial cells. Eur. J. Cell Biol. 88, 669-684 (2009).

[61] Rigort, A. et al. Micromachining tools and correlative approaches for cellular cryoelectron tomography. J. Struct. Biol. 172, 169-179 (2010).

[62] Le Gros, M. A., McDermott, G., Uchida, M., Knoechel, C. G. \& Larabell, C. A. Highaperture cryogenic light microscopy. J. Microsc. 235, 1-8 (2009).

[63] Kaufmann, R., Hagen, C. \& Grünewald, K. Fluorescence cryo-microscopy: current challenges and prospects. Curr. Opin. Chem. Biol. 20, 86-91 (2014).

[64] Hirschfeld, V. \& Hübner, C. G. A sensitive and versatile laser scanning confocal optical microscope for single-molecule fluorescence at 77 K. Rev. Sci. Instrum. 81, id. 113705 (2010).

[65] Marquardt, E., Le, J. \& Radebaugh, R. Cryogenic material properties database. 11th International Cryocooler Conference (2000). 
[66] Henriques, R. et al. QuickPALM: 3D real-time photoactivation nanoscopy image processing in imagej. Nat. Methods 7, 339-340 (2010).

[67] Schneider, C. A., Rasband, W. S. \& Eliceiri, K. W. Nih image to imagej: 25 years of image analysis. Nat. Methods 9, 671-675 (2012).

[68] Enderlein, J., Toprak, E. \& Selvin, P. R. Polarization effect on position accuracy of fluorophore localization. Opt. Express 14, 8111-8120 (2006).

[69] Trevoy, D. J. Rate of evaporation of glycerol in high vacuum. Ind. Eng. Chem. 45, 2366-2369 (1953).

[70] Wolter, S. et al. rapidSTORM: accurate, fast open-source software for localization microscopy. Nat. Methods 9, 1040-1041.

[71] Patterson, G. H. \& Lippincott-Schwartz, J. A photoactivatable GFP for selective photolabeling of proteins and cells. Science 297, 1873-1877 (2002).

[72] van de Linde, S. et al. Direct stochastic optical reconstruction microscopy with standard fluorescent probes. Nat. Protoc. 6, 991-1009 (2011).

[73] Mortensen, K. I., Churchman, L. S., Spudich, J. A. \& Flyvbjerg, H. Optimized localization analysis for single-molecule tracking and super-resolution microscopy. Nat. Methods 7, 377-381 (2010).

[74] Förster, T. 10th spiers memorial lecture. transfer mechanisms of electronic excitation. Discuss. Faraday Soc. 27, 7-17 (1959).

[75] Dale, R. E., Eisinger, J. \& Blumberg, W. E. The orientational freedom of molecular probes. The orientation factor in intramolecular energy transfer. Biophys. J. 26, 161193 (1979).

[76] Erdelyi, M. et al. Correcting chromatic offset in multicolor super-resolution localization microscopy. Opt. Express 21, 10978-10988 (2013).

[77] Pertsinidis, A., Zhang, Y. \& Chu, S. Subnanometre single-molecule localization, registration and distance measurements. Nature 466, 647-651 (2010).

[78] Ghosh, S. et al. Photoluminescence of carbon nanodots: Dipole emission centers and electron-phonon coupling. Nano Lett. 14, 5656-5661 (2014). 
[79] Patra, D., Gregor, I. \& Enderlein, J. Image analysis of defocused single-molecule images for three-dimensional molecule orientation studies. J. Phys. Chem. A 108, 6836-6841 (2004).

[80] Rothemund, P. W. K. Folding dna to create nanoscale shapes and patterns. Nature 440, 297-302 (2006). 


\section{List of Figures}

$1 \quad$ PSF of a conventional light microscope . . . . . . . . . . . . . . . . . . 4

$2 \quad$ Microscope resolution criteria $\ldots \ldots \ldots \ldots \ldots$. . . . . . . . . . . . . . .

3 Cryostat design . . . . . . . . . . . . . . . . . . . . . 13

4 Thermal conductivity of various materials . . . . . . . . . . . . . . . . . . . 14

5 Cryo-fluorescence microscope $\ldots \ldots \ldots$

$6 \quad$ Microscope controlling software. . . . . . . . . . . . . . . . 17

7 Cryostat cooling test . . . . . . . . . . . . . . . . . . 18

8 Lateral sample drift . . . . . . . . . . . . . . . . . . . . . . . . 20

$9 \quad$ Objective comparison for imaging quality . . . . . . . . . . . . . . . . . . 22

10 Objective photon collection efficiency with respect to NA . . . . . . . . . . . 23

11 Background comparison between normal coverslip and fused silica coverslip . 25

12 Cleaning procedure for silica coverslip . . . . . . . . . . . . . . . . . 26

13 Silica coverslip contamination by plasma cleaning . . . . . . . . . . . . . 27

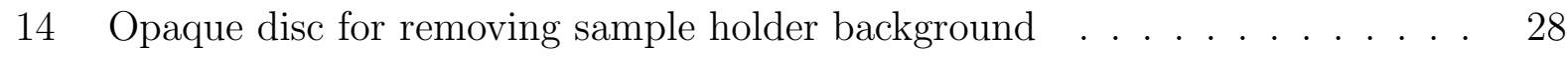

15 Objective-introduced background $\ldots \ldots \ldots$. . . . . . . . . . . . . . . 29

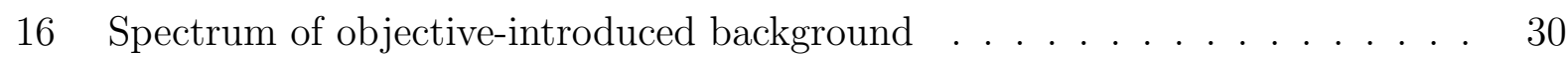

17 Iris filtering at the image plane . . . . . . . . . . . . . . . . . . . 31

18 Single molecule imaging at $89 \mathrm{~K}$. . . . . . . . . . . . . . . . . . 32

19 AFM image of silica coverslip surface . . . . . . . . . . . . . . . . . . 34

20 DNA rods on silica coverslip . . . . . . . . . . . . . . . . . . . . 35

21 PVA as mounting medium . . . . . . . . . . . . . . . . . . 36

22 Water crystallization by gradual cooling . . . . . . . . . . . . . . . 36

23 Sample transfer at CT . . . . . . . . . . . . . . . . . . . . . . 38

24 Nitrogen purging on the optical window . . . . . . . . . . . . . . . . 39

25 Piping system for gas supply . . . . . . . . . . . . . . . . . . . . 41

26 Photobleaching of Atto647N molecules at $294 \mathrm{~K}$ and $89 \mathrm{~K}$. . . . . . . . . . . 44

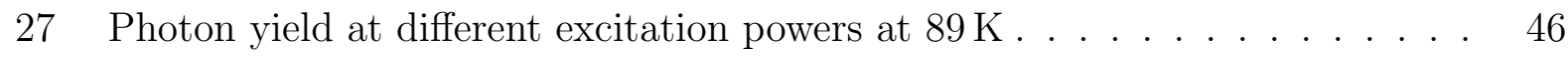

28 Blinking of Alexa647 molecules . . . . . . . . . . . . . . . . . . . . 48

$29 \quad$ Atto 488 molecules in vacuum $\ldots \ldots \ldots \ldots$. . . . . . . . . . . . . . . . 49

$30 \quad$ Stochastic photo-switching of Atto488 molecules at $89 \mathrm{~K}$. . . . . . . . . 50

31 Emission spectrum of organic dyes in vacuum at $89 \mathrm{~K}$. . . . . . . . . . . . 52

32 Lateral drift correction and single molecule localization . . . . . . . . . . . . 55

33 Comparison of localization precision between $89 \mathrm{~K}$ and $294 \mathrm{~K}$. . . . . . . . 56

34 Spectra of Atto488 and Atto674N dyes . . . . . . . . . . . . . . 58 
35 Chromatic offset of the cryo-imaging system . . . . . . . . . . . . . . . 59

36 Spectra of Atto647N and Atto490LS dyes . . . . . . . . . . . . . . . . . 60

37 Schematic of linearly polarized excitation and detection of two molecules. . . 63

$\begin{array}{llll}38 & \text { Experimental setup for polarization microscopy } & \ldots\end{array} \ldots$. . . . . . . . . . . . 66

39 Camera magnification adjustment . . . . . . . . . . . . . . . . . . . . . . . . 67

40 Camera alignment $\ldots \ldots \ldots$. . . . . . . . . . . . . . . . . . 67

$41 \quad$ Background fluctuation in polarization microscopy . . . . . . . . . . . . . . 68

42 Polarized excitation and detection of single molecules . . . . . . . . . . . . . 69 


\section{LIST OF MOVIES}

Movie 1 Photobleaching of Atto647N molecules at $294 \mathrm{~K}$

Atto647N molecules were spin-coated onto a silica coverslip. The sample was imaged at $294 \mathrm{~K}$ under irradiance of $\sim 300 \mathrm{~W} / \mathrm{cm}^{2}$ using a $637 \mathrm{~nm} \mathrm{CW}$ laser. Images were taken at $0.5 \mathrm{~Hz}$ with 100 EM-gain.

Movie 2 Photobleaching of Atto647N molecules at $89 \mathrm{~K}$

Atto647N molecules were spin-coated onto a silica coverslip. The sample was imaged at $89 \mathrm{~K}$ under irradiance of $\sim 300 \mathrm{~W} / \mathrm{cm}^{2}$ using a $637 \mathrm{~nm} \mathrm{CW}$ laser. Images were taken at $1 \mathrm{~Hz}$ with 100 EM-gain.

Movie 3 Influence of vacuum and CT on the blinking behaviour of Alexa647 molecules Alexa647 molecules were spin-coated on a silica coverslip. Movies were taken in the cryostat with $50 \mathrm{~ms}$ exposure time and 30 EM-gain in three conditions: (i) air, $293 \mathrm{~K}$; (ii) vacuum, $293 \mathrm{~K}$; (iii) vacuum, $89 \mathrm{~K}$.

Movie 4 Blinking of Atto647N molecules at $89 \mathrm{~K}$

Atto647N molecules were imaged on the silica coverslip at $89 \mathrm{~K}$ with $50 \mathrm{~ms}$ exposure time and 100 EM-gain.

Movie 5 Blinking of Cy5 molecules at $89 \mathrm{~K}$

Cy5 molecules were imaged on the silica coverslip at $89 \mathrm{~K}$ with $10 \mathrm{~ms}$ exposure time and 100 EM-gain.

Movie 6 Cryo-STORM with Atto488 molecules

Atto488 molecules were imaged in vacuum at $89 \mathrm{~K}$ under $473 \mathrm{~nm}$ excitation at power density of $\sim 0.75 \mathrm{~kW} / \mathrm{cm}^{2}$. Movie was taken with $50 \mathrm{~ms}$ exposure time and 100 EM-gain.

Movie 7 Stochastic photoswitching of Alexa488 molecules

Alexa488 molecules were imaged in vacuum at $89 \mathrm{~K}$ under $473 \mathrm{~nm}$ excitation at power density of $\sim 0.75 \mathrm{~kW} / \mathrm{cm}^{2}$. Movie was taken with $50 \mathrm{~ms}$ exposure time and 100 EM-gain. 
Movie 8 Stochastic switching at different excitation powers

The excitation power of movie 6 was reduced from $\sim 0.75 \mathrm{~kW} / \mathrm{cm}^{2}$ to $\sim 0.2 \mathrm{~kW} / \mathrm{cm}^{2}$. Movie was taken with $50 \mathrm{~ms}$ exposure time and 100 EM-gain.

Movie 9 Polarized excitation and detection of single molecules

Single Atto647N molecules were excited at $89 \mathrm{~K}$ with linearly polarized light. The polarization direction was rotated at a step size of $6 \mathrm{deg}$, and images were taken through a PBS with $5 \mathrm{~s}$ exposure time. 


\section{ABBREVIATIONS}

AFM atomic force microscopy

AOI angle of incidence

AU Airy unit

BSA bovine serum albumin

CCD charge-coupled device

CLEM correlative light and electron microscopy

CMOS complementary metal-oxide semiconductor

CNP carbon nano particle

cryo-EM cryo-electron microscopy

cryo-FM cryo-fluorescence microscopy

CT cryogenic temperature

CW continuous wave

dSTORM direct stochastic optical reconstruction microscopy

EM electron microscopy

EM-gain electron multiplying gain

FCS fluorescence correlation spectroscopy

FLIM fluorescence-lifetime imaging microscopy

FM fluorescence microscopy

FOV field of view

fPALM fluorescence-PALM

FP fluorescent protein

FRET Förster (fluorescence) resonance energy transfer

GFP green fluorescent protein

HILO highly inclined and laminated optical sheet 
ISM image scanning microscopy

LHe liquid helium

LN2 liquid nitrogen

LP long pass

LSS long Stokes shift

NA numerical aperture

NSOM near-field scanning optical microscopy

OFC oxygen-free copper

PA-FP photoactivatable fluorescent protein

PALM photoactivated localization microscopy

PBS polarizing beam splitter

PMT photomultiplier tube

PSF point spread function

PVA polyvinyl alcohol

RCM re-scan confocal microscopy

RT room temperature

s/b signal-to-background

$\mathbf{s} / \mathbf{n} \quad$ signal-to-noise

SIM structured illumination microscopy

sm-FM single molecule fluorescence microscopy

SMLM single molecule localization microscopy

SPAD single photon avalanche detector

SSIM saturated structured illumination microscopy

STED stimulated emission depletion

STORM stochastic optical reconstruction microscopy

TIRF total internal reflection fluorescence microscopy 
UV ultraviolet

WD working distance 


\section{ACKNOWLEDGMENTS}

I would like to thank all the people who have provided precious supports in this project.

Foresighted supervisors:

Prof. Jörg Enderlein for his sharp insight into the cryo-FM field, and for his blueprint of this project.

Prof. Holger Stark for his decisive suggestion to decouple the sample from the unstable cooling source.

Prof. Kai Tittmann for his precious perspectives on the possible applications of the cryo-system in structural biology.

Supportive scientific advisers:

Dr. Ingo Gregor for his constant readiness for providing theoretical and practical instructions in all directions.

Dr. Martin Wenderoth and Dipl. Ing. Bernhard Spicher (IV. Institute of Physics, Georg-August University Göttingen) for their patient instructions and feedbacks on the mechanical design of the cryostat.

Prof. Iwan Schaap (Heriot Watt University in Edinburgh) for his precious advices on the stabilization of the cryostat, and for assisting with the AFM experiment.

Dr. Olaf Schulz (PicoQuant GmbH, Berlin) for his tips on the optical design of the imaging system.

Dr. Qui Van for his advices on biological samples.

Talented workshops:

Dieter Hille and co-workers in the mechanical workshop for their brilliant capability of bringing the cryostat from drawings to reality.

Dr. Karl Lautscham, Markus Schönekeß and co-corkers in the electronic workshop for constructing the temperature sensor system in the cryostat. 
Last but not least, all the other group members, especially:

Simon Stein for his great contribution to the simulation and data analysis, and for the inspiring discussions.

Narain Karedla for sharing his insight into the single molecule microscopy theory and experiment.

Melanie Dannemeyer for providing her valuable experiences in the preliminary work of cryo-imaging.

This work was funded by the DFG Cluster of Excellence "Center for Nanoscale Microscopy and Molecular Physiology of the Brain" (CNMPB), and by a grant of the Deutsche Forschungsgemeinschaft (DFG, SFB 860, project A6). 


\section{EDUCATION / RESEACH EXPERIENCE}

2013.4 - present

Ph.D. candidate in Physics, Georg-August University Göttingen,

\section{Germany}

Thesis: Single-molecule cryo-fluorescece microscopy

Supervisor: Prof. Dr. Jörg Enderlein

2011.9 - 2012.12 Master of Science in Biophysics, Georg-August University Göttingen, Germany

Thesis: FPGA Based Fast Feedback Control for Nanometer Precise Surface

Detection in Optical Trapping Experiments

Supervisor: Prof. Dr. Iwan Schaap

2009.2 - 2011.2 Research assistant, Max-Planck Institute for Dynamics and SelfOrganization, Göttingen, Germany

Topic: In-vitro model system for actin-based motility

Supervisor: Prof. Dr. Eberhard Bodenschatz and Dr. Azam Gholami

2008.9 - 2011.7 Bachelor of Science in Biophysics, Georg-August University Göttingen, Germany

Thesis: Interference Effects on the Axial Detection Signal in Optical Traps

Supervisor: Prof. Dr. Iwan Schaap

\section{PUBLICATIONS}

Chizhik, A., Stein, S., Dekaliuk, M. O., Battle, C., Li, W., Huss, A., Platen, M., Schaap, I. A. T., Gregor, I., Demchenko, A. P., Schmidt, C. F., Enderlein, J. \& Chizhik, A. I. Super-resolution optical fluctuation bio-imaging with dual-color carbon nanodots. Nano Lett. 16, 237-242 (2015).

Li, W., Stein, S., Gregor, I.\& Enderlein, J. Ultra-stable and versatile widefield cryo-fluorescence microscope for single-molecule localization with sub-nanometer accuracy. Opt. Exp. 23, 37703783 (2015).

Bodensiek, K., Li, W., Sánchez, P., Nawaz, S. \& Schaap, I. A. T. A high-speed vertical optical trap for the mechanical testing of living cells at piconewton forces. Rev. Sci. Instrum. 84, 113707 (2013).

\section{CONFERENCE TALLKS}

2015.4 Göttingen Focus on Microscopy (FOM)

2015.3 Berlin DPG Spring Meeting

2015.1 Paris Quantitative Bioimaging (QBI)

2014.9 Berlin 20th International Workshop on Single Molecule Spectroscopy and Ultra Sensitive Analysis in the Life Sciences - Best Student Talk Award 